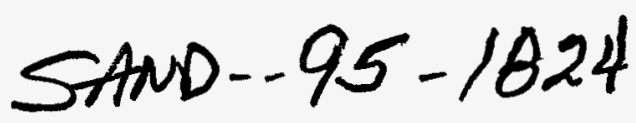

\title{
Challenging and Improving Conceptual Models for Isothermal Flow in Unsaturated, Fractured Rock Through Exploration of Small-Scale Processes
}

\author{
R.J. Glass, M.J. Nicholl, and V.C. Tidwell \\ Subsurface Flow and Transport Processes Laboratory \\ Sandia National Laboratories \\ Albuquerque, New Mexico \\ RECEIVED \\ JUL 311996 \\ OSTI
}

\begin{abstract}
Over the past several years, we have performed experimental studies focused on understanding small-scale flow processes within discrete fractures and individual matrix blocks; much of the understanding gained in that time differs from that underlying the basic assumptions used in effective media representations. Here we synthesize the process level understanding gained from our laboratory studies to explore how such small-scale processes may influence the behavior of fluid flow in fracture networks and ensembles of matrix blocks at levels sufficient to impact the formulation of intermediate-scale effective media properties. We also explore, by means of a thought experiment, how these same small-scale processes could couple to produce a large-scale system response inconsistent with current conceptual models based on continuum representations of flow through unsaturated, fractured rock. Based on our findings, a number of modifications to existing dual permeability models are suggested that should allow them improved applicability; however, even with these modifications, it is likely that continuum representations of flow through unsaturated fractured rock will have limited validity and must therefore be applied with caution.
\end{abstract}

\section{Introduction}

Fluid flow in unsaturated, fractured rock is currently being studied with respect to the siting of the nation's first high-level radioactive waste repository. Spatial scales associated with this problem vary from meters to kilometers, with temporal scales ranging from months to tens of thousands of years. Because such scales often preclude direct physical exploration of system response and detailed site characterization, we are forced to use our understanding (or misunderstanding) of the underlying physical processes to predict large scale behavior. For this reason, it is essential that conceptual models used as the basis for prediction be firmly grounded in physical reality.

The unique aspect of flow and transport through unsaturated, fractured rock is that two systems (fractures and matrix) exhibiting vastly different hydraulic behavior are present within the same domain. In both systems, assuming thermal-mechanical-geochemical effects to be negligible, fluid flow is primarily governed by capillary, gravity, and viscous forces, the effects of which are relatively well understood in unsaturated porous media (i.e., matrix). As a result, virtually all of our process-related uncertainty is associated with the incorporation of fractures into the system. This uncertainty is unfortunate, as the physical nature of fractures (relatively large, open, connected void spaces) implies that they will dominate system hydraulics whether they are flowing (conduits) or not (barriers).

We are confronted with two primary sets of questions concerning flowing and non-flowing fractures: 
1) Under what conditions will fractures conduct flow? When they do flow, how fast, over what distance, and how will matrix interaction affect fracture flow? Can properties for individual fractures be modeled and measured? Can "effective" property models for the fracture network be formulated? If so, at what scales?

2) When fractures do not flow, how do they constrain flow in the matrix (capillary barriers)? Can these constraints be incorporated into "effective" property models for the fractured matrix? In addition to process-related uncertainty embodied in these sets of questions, we are also faced with limited data on material properties (hydraulic and geometric) at all scales. This lack of data makes the understanding of fundamental underlying processes all the more important in order to place bounds on expected behavior.

A number of authors have contributed to the development of conceptual models for flow and transport through unsaturated fractured rock (e.g., Wang and Narasimhan, 1985; Evans and Nicholson, 1987; Pruess and Wang, 1987; Peters and Klavetter, 1988). However, the paucity of experimental data forced these, and subsequent authors, to make many assumptions that have oversimplified the influence of fractures (Eaton et al., 1990). In an effort to explore the validity of some of these assumptions, a laboratory research program was initiated at Sandia National Laboratories in 1991 (for a full description see Glass and Tidwell, 1991). The laboratory research program is directed toward developing and testing the validity of macroscopic, continuum-based models and supporting effective property models because of their widespread utility within the context of the Yucca Mountain Project. In order to pursue these goals, physical experimentation is integrated with conceptual model formulation and mathematical modeling.

In this paper, we consider results of our laboratory experiments that elucidate isothermal, smallscale processes within the plane of single fractures and within discrete matrix blocks. Our intent is not to review, but to illustrate some of the implications of these recent results with respect to flow in fracture networks, ensembles of matrix blocks, interaction between fractures and matrix, and consequently the formulation of intermediate-scale effective media properties. To stimulate "crossscale" discussion among researchers on this project and with practitioners responsible for applying models to assess the performance of the natural barriers at Yucca Mountain, we also present a thought experiment in which small-scale processes interact to create a large-scale system response that is very different than that predicted by current unsaturated flow modeling. Implications of the thought experiment are far reaching and demonstrate the need within the Yucca Mountain Project of an integrated, multi-scale approach for model development and validation.

\section{Small-Scale Processes in Single Fractures and Individual Matrix Blocks}

Ultimately we are concerned with flow and transport at the large-scale, or mountain-scale as in the case of the Yucca Mountain Project (see figure 1). Currently formulated continuum models for flow in unsaturated, fractured rock require the division of the rock formation into a number of smaller scale grid-blocks for which effective properties are defined (e.g., Arnold et al., 1994, see figure 2). Properties of individual grid-blocks necessarily integrate over sub-scale processes and features. Proper application of continuum models requires that the uncertainty associated with the underlying assumptions at the grid-block-scale be evaluated, and if possible reduced. It is therefore necessary to bound the validity of effective continuum conceptualizations and within those bounds, develop improved conceptual models for defining grid-block-scale effective properties through consideration of sub-scale processes. In this section, we explore recent advances in understanding the small-scale processes which govern unsaturated flow in discrete fractures and individual matrix blocks and that must be properly integrated into larger grid-blockscale effective media properties.

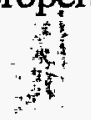




\section{DISCLAIMER}

Portions of this document may be illegible in electronic image products. Images are produced from the best available original document. 


\section{Discrete fracture processes}

In fractures, as in porous media, geometry of the connected fluid phase will control the system hydraulic properties at Darcian flow velocities. Under fully saturated conditions, the hydraulic properties of an individual fracture in any given direction will be defined by the aperture geometry. However, for unsaturated flow, geometry of the flowing phase (phase structure) will differ from the aperture geometry (see figure 3). It is therefore necessary to carefully understand the processes that control distribution of the phases along the fracture plane.

For most natural gradient conditions in unsaturated, fractured rock, viscous forces will be small with respect to capillary and/or gravitational forces. Under such conditions, wetting-phase invasion of a horizontal fracture plane is controlled by capillary forces and phase accessibility. Complicated phase structures containing significant entrapped air form regardless of whether water enters the fracture from the fracture edge (Nicholl and Glass, 1994) (see figures $4 a, b$ ); or from the matrix via contact points (Glass and Norton, 1992) (see figure 5). For both these situations, significant hysteresis in the pressure-saturation relations and phase structure have been measured (see figure 6). In experiments demonstrating air entrapment during in-plane wetting-phase invasion, fracture satiation was reached at saturations $(S)$ of 0.6 to 0.7 , with an associated increase in flow channelization (see figure 7) and decrease in fracture relative permeability $\left(\mathrm{k}_{\mathrm{r}}\right)$ to less than 20\% of the saturated value (Nicholl and Glass, 1994) (see figure 8).

Invasion percolation theory with phase trapping in two-dimensional networks (e.g., Wilkinson and Willemsem, 1983; Glass, 1993) suggests a well defined percolation threshold pressure exists for each phase when fractures are much larger than the correlation length of the aperture field (where the field behaves as a random network). At pressures above this threshold, one phase spans the system and fully entraps the other; this preclusion of a bi-continua creates a satiated condition (see figure 9). For fractures smaller than the aperture correlation length, the percolation threshold pressure will be a function of aperture structure, and for highly anisotropic aperture fields, a directional bi-continua can exist (e.g., Pruess and Tsang, 1990). Relative permeability as a function of saturation is therefore expected to approximate a step function; finite above the percolation threshold, and zero below, with an abrupt transition region. Percolation theory also predicts a fractal nature for the entrapped structure; the existence of phase structure at multiple scales implies a scale dependence for satiated fracture permeability (inverse with scale). Such scale dependence is expected even where the correlation length of the fracture aperture field is much smaller than the fracture size (fractal structure of the aperture field is constrained below this length).

The dissolution or evolution of entrapped phase within the fracture alters phase structure (see figure 10). Gas depleted water dissolves air from the entrapped phase, increasing fluid saturation (and relative permeability) while supersaturated water evolves gases with opposite results. The gas exchange process proceeds at a rate dependent on the fluid flow rate, gas diffusion rate, and the gas concentration gradients within the water surrounding the entrapped bodies. These quantities in turn are defined by the phase structure and position within the flow field. Specific changes to the phase structure from gas dissolution are driven by accessibility concerns coupled with the initial entrapped phase structure and hence will not necessarily drive the system towards a unique equilibrium structure (e.g., Glass and Nicholl, 1995) (see figure 11).

When fluid supply is insufficient to support gravity-driven saturated flux, gravity-driven "fingers" are expected to form in non-horizontal fractures (see figure 12). Resulting wetted structures will be relatively compact and oriented along the gravitational gradient (Glass, 1990). In an initially dry fracture, gravity-driven instability is triggered by reversal of the capillary gradient at the cessation of ponded infiltration (see figure 13). Linear stability theory (Saffman and Taylor, 1958) has 
been used to analyze the breakup (instability) of a planar front advancing downward in porous media and smooth walled fractures (i.e., Hele-Shaw cells). However, since fluid is never supplied uniformly in nature, fingers resulting from the cessation of ponded infiltration in rough-walled fractures (see figures 12-17) will be primarily defined by inflow boundary irregularities (finite amplitude perturbations) yielding finger widths different from those predicted by linear theory in either initially dry (Nicholl et al., 1992, 1994) or pre-wetted fractures (Nicholl et al., 1993b).

Instability of an advancing planar front is not required to form gravity-driven fingers. Where steady flux to a fracture is less than the gravity-driven saturated flux, fingers are expected to form (Nicholl et al., 1994); initiating from local heterogeneities (point connections) along the fracture's upper boundary (see figures 18-20). Point sources abound in individual fractures and fracture networks: wetted regions and contact points where water will enter the fracture from the matrix; low points along fracture intersections; irregularities in water inflow to a fracture or fracture network. The velocity (see figure 21) and width (see figure 22) of individual fingers initiated from single point sources are dependent on supply rate, fracture conductivity, and fracture inclination (Nicholl et al., 1993a). Steady supply does not necessarily imply steady flow, as desaturated regions behind advancing finger tips have been observed to display intermittent and possibly chaotic behavior (see figure 23).

Initial moisture content within a fracture has a significant impact on finger behavior. Fingers tend to follow existing wetted structure (see figure 24), leading to persistent pathways for successive events (Nicholl et al., 1993b). In contrast to observations in porous media (Diment and Watson, 1985), initial moisture at the residual value does not necessarily stabilize fracture flow (see figure 25) (Nicholl et al., 1993b). Under uniformly moist initial conditions, gravity-driven fingers are observed to be faster (see figure 26) and narrower (see figure 27) than in an identical, but initially dry system. Thus, for unsaturated flow in non-horizontal fractures, gravity-driven finger structures are expected, as long as fracture width exceeds that of the finger (a function of supply rate, inclination, and fracture permeability).

We can assemble this understanding to postulate the behavior of relative permeability for single fractures that are significantly larger than their aperture correlation lengths and in the case of vertical fractures, the expected minimum finger width. At horizontal orientations, relative permeability will be a discontinuous function of saturation; zero at pressures below the percolation threshold and then jump to the satiated value. Fracture satiation, and hence permeability, will be a function of wetting history and boundary conditions, as well as an inverse function of fracture extent. If saturations above the percolation threshold are reached through gas dissolution, then relative permeability will follow a power law relationship $\left(k_{r}-S^{n}\right)$ where $n$ likely equals 4 or higher (Glass and Nicholl, 1995) (see figure 8). In the vertical case, the influence of gravity is expected to produce an oriented phase structure that fully samples the aperture distribution; under such conditions, relative permeability will follow fracture saturation $\left(k_{\Gamma} \sim S\right)$ with no fixed lower limit. The compact nature of fingers implies that fracture satiation, and thus satiated relative permeability, may be an increasing function of gravity; further exacerbating permeability differences between vertical and horizontal fractures (see figure 28). Also in contrast to horizontal fractures, gravity-induced anisotropy due to fingers suggests that simultaneous vertical flow of both phases will occur across a wide range of saturations, while horizontal flow may be restricted. As a further complication, relative permeability for all fracture inclinations will be a hysteretic function of pressure.

\section{Discrete matrix block processes:}

In fractured systems, the porous matrix is composed of a series of blocks either fully or partially separated from their neighbors by bounding fractures. In unsaturated, fractured systems, the 
matrix acts either as a buffer to flow when fractures are conducting, or as the primary flow path when they are not. For both of these behavioral modes, discrete matrix block processes are controlled by the external connections to other blocks or fractures (the fracture wetted structure) and the internal block property field.

In its buffer mode, matrix blocks imbibe (supply) water from (to) fractures depending on the pressure gradients existing between the fracture and the matrix. The pressure gradient is a time dependent function of matrix block and fracture properties, flow geometry, and the initial conditions (or pressure history). For imbibition, the ratio of flux through the fracture to flux from the fracture into the matrix controls the gross behavior of the system. When this ratio is small (high permeability and/or low initial saturation of the matrix) the penetration of water into the matrix can keep pace with the penetration of water down the fracture (see figure 29). Conversely, when this difference is large (low permeability and or high initial saturation of the matrix) fracture flow is rapid accompanied by slow, relatively uniform wetting of the matrix normal to the fracture (see figure 30) (also see analysis by Nitao and Buscheck 1991; Martinez, 1988).

As discussed in the previous section on discrete fracture processes, it is likely that the formation of complicated phase structures within the bounding fractures will preclude uniform wetting of the periphery of matrix blocks. In general, matrix imbibition will be proportional to wetted surface area; however, the associated functional relation will not be linear (1-D flow from a fully wetted fracture plane will transition to 2- or 3-D as the wetted area decreases thus increasing the imbibition rate per fracture cross-sectional area) (Tidwell et al., 1995). In addition, the overall matrix block geometry influences imbibition through both the surface area to volume ratio and the degree of convergence/divergence imparted to the within block unsaturated flow field (Zimmerman et al., 1990; Zimmerman and Bodvarsson, 1995). In general, geometries characterized by high surface area to volume ratios will have higher imbibition rates (high surface area and low flow convergence) than those with lower ratios. If the time scale for the redistribution of water into and within the matrix following a transient water pulse within the fracture is longer than the time between the pulses, non-uniform saturation encountered spatially within the fracture and along the matrix surface will lead to the persistence of fracture wetted structure. The higher local initial saturation within the matrix leads to a much reduced gradient driving flow and hence further reduced matrix imbibition in these zones.

The presence of altered permeability zones along the edge of matrix blocks (e.g., fracture coatings, matsix alteration) influences matrix imbibition (Chekuri et al., 1994; Thoma et al., 1992). However, detailed knowledge of the internal matrix block properties and their precise spatial variation has little impact on the modeling of overall matrix imbibition. In comparing a physical matrix imbibition experiment with numerical simulations using five different hydraulic property fields of increasing complexity (see figures 31-33) it was found that relatively little detail is required to capture salient flow features (Glass et al., 1994). In fact, the effective block hydraulic parameters were noted to closely predict the mean position of the wetting front (see figure 33). Similarly, the matrix sorptivity (a hydraulic property which quantifies matrix imbibition processes) exhibits orders of magnitude less variability than the hydraulic conductivity (Tidwell et al., 1995) (See figure 34).

The effective properties of a discrete block, are dependent on its internal variability in some average sense. Characterization of this variability is a formidable task as these effective properties are scale dependent. According to permeability measurements made on volcanic tuffs from Yucca Mountain (see figure 35) the mean and variance dramatically decrease with increasing sample support (volume) (see figure 36), while the correlation length scale increases in proportion to the characteristic length of the measurement (see figure 37) (Tidwell, 1995). For these volcanic tuffs, up-scaling rules needed to move from the core sample scale to the discrete matrix block scale must account for the prevalent bi-modal permeability distribution in which one mode is associated 
with the groundmass and the other mode associated with the more porous fraction of the medium (i.e., pumice, lithics, and lithophysae). Because of these two modes, the effective permeability will scale as a function of the groundmass/porous fraction ratio and the interconnectedness or networking of the porous fraction. Where this ratio and networking are high, the effective saturated permeability will significantly exceed the arithmetic average of the core scale measurements. Where the ratio and networking are low, the permeability will approach that of, or below the groundmass. Finally, since air entrapment within matrix blocks is likely to occur whenever the largest pores in the medium are poorly connected across the system, satiated values of the permeability may be as much as an order of magnitude lower than the scaled saturated permeability depending on the satiated saturation and slope of the relative permeability curve.

\section{Implications for Formulation of Grid Block Scale Effective Media Properties}

At the grid block scale, composite-continuum models (e.g., Peters and Klavetter, 1988) require definition of a single effective media property that describes the combined behavior of both fractures and matrix (see figure $2 b$ ). The assumptions inherent in this approach (e.g., pressure equilibrium between fractures and adjacent matrix) make it most suitable for steady-state, uniform boundary condition flow. To model the highly non-equilibrium situations of importance for evaluating many scenarios for total system performance assessment at Yucca Mountain (see Barr et al., 1995), dual-continua models are presumed to be more applicable. Dual-permeability models (e.g., Kazemi and Gilman, 1993) require definition of separate effective media properties for the fracture network and ensemble of individual matrix blocks within each grid block, as well as a transfer function between the two continua (see figure 2d). For computational expediency, it is often assumed that flow through the matrix continua is negligible, allowing inter-block connections to be severed (dual-porosity model, Warren and Root, 1963) (see figure 2c). In the previous section we discussed current understanding of small-scale processes acting within discrete fractures and matrix blocks. Here we focus on dual-continua models and consider the impact of these processes on the relative permeability of fracture networks and ensembles of matrix blocks, and fracture-matrix interaction as required by these models.

\section{Fracture network permeability}

At this time, experimental and numerical investigations of flow through unsaturated, fractured rock performed above the scale of an individual fracture, have not been designed to consider the discrete fracture behavior discussed above. Thamir et al. (1993) conducted an experiment in a fractured block that was not designed to allow measurement of such fracture behavior. Kwicklis and Healy (1993) have numerically studied the permeability of a 2-D network of fractures; however, the pressure/saturation and relative permeability relations used for the fractures did not incorporate satiated limits, hysteresis, or gravity-driven fingering in non-horizontal fractures.

The primary uncertainty to be addressed regards potential averaging of discrete fracture properties by the network. It is unknown at this time if fracture networks will behave similar to 3-D porous media and smooth out flow heterogeneities that dominate 2-D systems such as individual fractures. If we assume that the fracture network is in some sense pervasive and fully averages the discrete behavior of individual fractures, we can use the directional behavior of fractures in the gravity field, as discussed above, to approximate at first order an anisotropic fracture network permeability. However, gravity-driven fingers tend to merge in individual fractures and thus, the possibility of finger confluence at larger scales within the fracture network itself must also be considered. Fracture intersections could cause the confluence of fingered flow (much as we see in the laboratory when the bottom boundary of a fracture is left open to the air) with the formation of 
much stronger, more conductive fingers below (see figure 38). By analogy to studies in porous media (Glass et al., 1989; Glass and Nicholl, 1996), assuming the system evolves to pressure potential equilibrium in the horizontal, hysteretic response of fractures during fingering would limit the transfer of fluid from the finger both within the fracture plane and through the matrix to other fractures (see figure 39).

\section{Matrix block ensemble permeability}

Matrix flow for an ensemble of blocks will ultimately depend on hydraulic connection of individual matrix blocks across the fractures (see figure 40-42). For dry fractures, connection between adjacent matrix blocks is controlled by the inter-block contact points. Limited contact area severely restricts connectivity, and hence, reduces permeability of the ensemble. In partially-saturated fractures, fracture wetted structure (as discussed above) defines connection between matrix blocks, introducing a tortuosity term for matrix-matrix communication much different than that suggested by Wang and Narasimhan (1985). Blocks will have much less connection across vertical fractures due to fingering in the fracture plane than across horizontal fractures where the connection will most likely be fractal due to entrapment of air. Since fractures display strong hysteretic response to changes in matrix pressure, fractures at all angles are likely to form complicated sets of capillary barriers greatly constraining the transmission of flow from one fracture to other fractures and the matrix beyond (see figure 41-42). The end result may allow formation of a preferential flow structure defined primarily by it's dynamic history rather than by heterogeneity in the material properties.

\section{Fracture-matrix interaction:}

Formulation of the transfer function embodying fracture-matrix interaction must include the local pressure gradient between the fracture and the matrix, matrix hydraulic properties (discussed above), and a term that defines the area across which the fracture and matrix continua communicate (i.e., connected wetted fracture area). The local pressure gradient and matrix properties are defined primarily by the initial saturation of the matrix. Where the matrix is near satiated, the pressure gradient and sorptivity will be small, hence little imbibition will occur. At any particular pressure, processes such as air entrapment and gravity driven fingering work to reduce wetted area within single fractures (see figure 43) and the fracture network, to fractions as low as 0.01 to 0.001 of the total fracture area. The transfer function under such conditions must account for the 2- to 3-D nature of matrix imbibition as well as the complex wetting history of both the matrix and fracture network. Armoring of fractures by mineral precipitates (Chekuri et al., 1994; Thoma et al., 1992) may work to reduce communication even further. From a combination of these reductions in permeability and wetted area, matrix imbibition may be reduced by as much as 4 to 5 orders of magnitude from what would be calculated using state of the art, dual continua models such as TOUGH2. Unfortunately, matrix imbibition has yet to be investigated under these combined expected conditions.

\section{Large-Scale System Behavior?}

A major limitation of current effective continuum models for flow through unsaturated, fractured rock, is that focusing of flow and the associated formation of rapid transport pathways can only occur if significant (and perhaps unphysical) heterogeneity is inserted within the system model (e.g., Bodvarsson et al., 1994; Arnold et al., 1994). Above, we have discussed mechanisms that would permit formation of significant transport pathways within individual unsaturated fractures and fracture networks. These mechanisms initiate flow features smaller than the typical grid-block- 
scale (where effective properties are defined) that may network and form connected flow conduits at larger scales. The creation of these features within an unsaturated, fractured rock mass would be primarily process driven, and could occur in a system showing no heterogeneity at the grid-blockscale. In this section we use a simple thought experiment to demonstrate our point; noting that this is only one of many possible combinations of assumptions that must be considered. We then provide observations from natural systems which offer evidence consistent with the outcome of this thought experiment.

\section{Thought experiment}

Consider a fractured rock unit of low matrix permeability/porosity that is dissected by a well connected fracture network with no specific preferential orientation. We assume that individual fractures exhibit an uninterrupted spatial extent that is both larger than the expected finger width and significantly larger than the aperture correlation lengths. As an initial condition, we assume that the fractures are dry and that the matrix is in a satiated state throughout the domain. The thought experiment begins by introducing steady flow at numerous discrete locations in the fracture network that are distributed along a horizontal plane passing through the system (e.g., nonuniform leakage from a perched zone or steady recharge focused into point sources by heterogeneity along a material boundary). The localized flow rates at the top input boundary are assumed to be high enough that the local potential gradient through the surrounding satiated matrix is unable to conduct the steady flow.

Gravity-driven fingers develop in inclined fractures near the input locations. Due to the hysteretic response of the fractures, once formed, these fingers persist. Fingered flow reduces interactions with the matrix blocks and fracture hysteretic response constrains the ability of the matrix to transmit fluid to other blocks and fractures. Thus, dissipation of the fingers via matrix imbibition and flow is severely limited. Heterogeneities in the fracture system (aperture, surface wettability, horizontal and sub-horizontal fractures) act to alter finger path, increasing the probability of finger contact and merger. Upon intersecting a large aperture fracture that the finger cannot enter, the finger will be deflected by the capillary barrier and stop at its lowest point. Other fingers within the catchment zone of this barrier will merge above the barrier and feed into a confluence zone above this low point. The pressure within this local tension-satiated confluence zone builds until the water entry pressure of the barrier fracture is reached. Flow then crosses into the barrier fracture at one or at most a small number of discrete points from which fingers form and continue downward tc the next barrier/confluence zone. The process of merger due to the barrier/confluence mechanism allows the focusing of distributed flow sources into a small number of strong flow paths through the unsaturated, fractured rock mass.

It is important to note that the system-scale preferential flow paths described here are determined by the networking of small-scale processes (gravity-driven fingering, hysteretic fracture response, and barrier/confluence mechanisms). While we have observed the individual processes in the laboratory, experiments have not been conducted to verify the postulated networking. However, assuming this to be correct, the formation of large-scale preferential flow paths does not require any particular media or fracture network heterogeneity structure to be present. Therefore, spatial location of the primary flow paths may move in response to some system perturbations (i.e., excavation induced alteration of the stress field, climate change, tectonic activity) while remaining invariant under others (i.e., weather patterns). However, once a structure forms, over time it may alter system properties through geochemical processes to determine either its persistence or extinction in time. As currently formulated, continuum based models would dissipate flow from the point sources along the top boundary of our thought experiment, unless focusing is forced by significant material heterogeneity. Arbitrary definition of preferential flow structures through heterogeneous material properties creates features that may be unphysical; that are not expected to respond to system perturbations in the same manner as those created by the coupling of small-scale 
flow processes as described in this thought experiment; and that may either over or under estimate the focused flux of fluid within the system. Furthermore, without the proper mechanism for advective transport, predictions for transport (including those incorporating dispersion, adsorption, ion exchange, etc.) will be based on an erroneous foundation.

\section{Reality of thought experiment}

Insufficient data are available at this time to refute our postulated large-scale system behavior; in fact, the outcome of our thought experiment is consistent with observations at a number of natural fractured, unsaturated rock systems. The presence of localized fracture flow within otherwise unsaturated media is commonly encountered throughout the western United States. Specifically, flowing fractures located $\sim 350 \mathrm{~m}$ below ground surface have been investigated at Rainier Mesa on the Nevada Test Site. Results suggest rapid fracture flow leading to travel times from the surface on the order of months to a few years (Russell et al., 1987). Similar studies are being performed on a series of flowing fractures located in a mining portal near the Apache Leap Site in Arizona (Bassett et al., 1994).

At Yucca Mountain, Nevada, saturation data suggest that many of the fractured welded units are at saturations above 0.6 at depth (A.L. Flint, personal communication, 11/29/94). Since satiated values for welded tuff have been found to range from 0.4 to 0.9 (E.M. Kwicklis, personal communication, 11/29/94) these units may be very near or at satiated saturations, thus greatly limiting matrix absorption of fracture flow as we assumed in our thought experiment. Geochemical data $\left(\mathrm{Cl}^{36}\right.$, tritium, $\left.\mathrm{C}^{14}\right)$ collected at Yucca Mountain to date have provided evidence for possible rapid movement of some water within the unsaturated fractured rock. Liu et al., (1995) reports bomb pulse chlorine $\mathrm{Cl}^{36}$ at depths of over $350-450 \mathrm{~m}$ below surface. Locally elevated levels of tritium (420-430 m below surface) and $\mathrm{C}^{14}$ (369-435 $\mathrm{m}$ below surface) also indicate the presence of modern water at depth at Yucca Mountain (I.W. Yang, personal communication, 11/29/94). While other causes for elevated concentrations of these elements are under evaluation, the fact that all three are found at depth is highly suggestive of rapid flow paths.

Currently accepted effective continuum models for flow in unsaturated, fractured rock do not support such rapid water movement without including extreme heterogeneity and boundary conditions. The combination of heterogeneity and boundary conditions could be considered to form a competing hypothesis to that proposed in onr thought experiment above. It is most likely, however, that heterogeneity, boundary conditions, and small-scale processes all combine to focus flow at the system scale. Because system response to perturbations (boundary conditions) will be dependent on the relative importance of heterogeneity and processes, it is important to resolve the significance of each. To test the relative importance of each in forming preferential flow structures within unsaturated fractured rock will require carefully designed physical experiments and numerical simulations at a variety of scales and sites.

\section{Conclusion}

In order to formulate conceptual models for flow and transport through unsaturated, fractured rock, we must consider the processes affecting system response at a variety of scales. Small-scale (time, space) experimentation and analysis provide fundamental understanding of flow and transport processes. Coupling of these processes in a complex physical system yields hypothesized system responses that can be tested through intermediate-scale physical experiments and numerical simulations that explicitly include small-scale processes. At large scales, observations of both the natural system of interest and natural analog systems must be explored in 
the context of both small- and intermediate-scale understanding, with an ultimate goal of firmly grounding predictive models in physical reality.

To date, this approach has been only partially implemented within the Yucca Mountain Project. Here we have synthesized our current understanding of small scale processes as determined from laboratory experimentation and hypothesized system responses at intermediate and large scales. As the hypothesized responses differ significantly from the effective media models currently used in performance assessment calculations, numerical simulations must be formulated and conducted to test these hypotheses. In the past, testing such hypothesis would not have been possible, as numerical tools capable of explicitly incorporating fractures at arbitrary angles into a participating matrix are only now becoming available (e.g., Zyvoloski et al., 1995). In addition, planned field tests for the ESF must be evaluated to determine if they are still well founded and capable of resolving these flow mechanism issues.

Our current understanding suggests several simple modifications to dual permeability models that can be made and evaluated with respect to model sensitivity:

- satiated permeability for a fracture network is anisotropic and given by the saturated value in the vertical direction and the satiated value in the horizontal (approximately 0.2 of saturated value)

- relative permeability of the fracture network is anisotropic and of the form illustrated in figure 28.

- matrix ensemble permeability for satiated fractures and matrix is anisotropic and given by the satiated value (order of magnitude reduction) times the cross-sectional fracture area open to flow (approximately 1.0 for vertical and 0.6 for horizontal)

- matrix ensemble permeability for satiated matrix and dry fractures is reduced dramatically to account for reduced cross-sectional area open to flow across the fracture and enhanced tortuosity. This will be anisotropic with less contact area for vertical fractures and more for horizontal fractures due to stress loading with depth, except possibly in the near field and thermally disturbed regions.

- fracture matrix interaction terms must be modified to account for reduction in fracture wetted area. This term also must be anisotropic with higher values for horizontal fractures and lower for vertical fractures.

Preliminary dual-permeability modeling with a reduction by two orders of magnitude in the fracture wetted area have shown significant influence on flow velocities and travel times (Ho, 1995). Implementation of the full list of modifications above will alter flow and transport substantially beyond that predicted by the preliminary study. However, even with this and the other modifications listed above implemented, it is highly questionable whether dual permeability models will be able to truly represent flow in unsaturated fractured rock for the many scenarios required by the Yucca Mountain Project without complete reconceptualization. Small-scale experiments suggest that the permeabilities of each continua, the fractures and the matrix, are coupled to create local behavior that is not easily represented at a larger grid-block-scale. This coupling cannot be fully accounted for in dual permeability model's interaction term. Fractures that are not flowing below the grid block scale form local capillary barriers to flow in the matrix continua. In addition, hysteretic response acting differentially in the fracture network within a single element cannot be represented. In the thought experiment we further extrapolate the essence of this small scale understanding past the intermediate to the large scale. Our end result hypothesizes the formation of preferential flow structures at a variety of scales and defined by dynamic history.

It must be emphasized that physical experimentation has not yet been designed or conducted to test our hypothesized intermediate- and large-scale system responses. Based on our experience in designing and conducting experiments, it is our view that such experimentation would not be overly difficult or expensive to undertake. It is also our view that since the implications of these hypotheses for the behavior of Yucca Mountain as a potential high-level waste repository are profound, that such tests must be designed and conducted in the future. Until such time, the ability 
of all continuum based numerical modeling to adequately predict flow and transport in fractured, unsaturated rock is questionable. In addition, since our current synthesis excludes the coupling of thermal, mechanical, and geochemical processes to the hydrologic processes, we contend that analyses in these other areas where hydrologic processes are important are also of questionable validity.

\section{Acknowledgments}

The authors would like to thank A.L. Flint, E.M. Kwicklis, and I.W. Yang of the USGS for providing preliminary data; G.E. Barr and M.L. Wilson of Sandia National Laboratories, J.H. Gauthier of Spectra Research, and R.W. Nelson of Intera for thoughtful review of an earlier less inclusive version of this paper (Glass et al., 1995); and S.R. Sobolik, S.J. Altman, and P.B. Davies of Sandia National Laboratories for their insightful review of this manuscript. This work was supported by the U.S. Department of Energy, Office of Civilian Radioactive Waste Management, Yucca Mountain Site Characterization Project Office, under contract DE-ACO494AL85000, WBS 1.2.5.4.6, WA-0040, and QAGR 1.2.5.4.6 Revision 0. All data referenced in this report are non-qualified. The data are archived under DTNs SNL19000000004.000, SNL19011590001.001, SNL19011590002.001, SNL19011590002.002, SNL19011590002.003, SNL19011590002.004, SNL19011590003.001,

SNL19011590003.002, and SNL19011590003.003. Data referenced in figures 3-10 and 41 have not been archived in the data management system at this time because associated experimental data sets have not been completed.

\section{References}

Arnold, B.W., S.J. Altman; T.H. Robey, R.W. Barnard, and T.J. Brown, " Unsaturatedzone fast-path flow calculations for Yucca Mountain groundwater travel time analyses (GWTT-94)", SAND95-0857, Sandia National Laboratories, 1995.

Barr, G.E., R.L. Hunter, E. Dunn, and A. Flint, "Scenarios constructed for nominal flow in the presence of a repository at Yucca Mountain and vicinity", SAND92-2186, Sandia National Laboratories, 1995.

Bassett, R.L., S.P. Neuman, T.C. Rasmussen, A. Guzman, G.R. Davidson, and C.F. Lohrstorfer, "Validation studies for assessing unsaturated flow and transport through fractured rock", NUREG/CR-6203, U.S. Nuclear Regulatory Commission, 1994.

Bodvarsson, G., G. Chen, and C. Wittwer, "Preliminary analysis of three-dimensional moisture flow within Yucca Mountain, Nevada", Proceedings of the Fifth Annual International Conference on High Level Radioactive Waste Management, 2038-2047, American Nuclear Society, Las Vegas, Nevada, May 22-26, 1994.

Chekuri, V.S, S.W. Tyler, and J.W. Fordham, "The role of fracture coatings on water imbibition into unsaturated tuff", Proceedings of the Fifth Annual International Conference on High Level Radioactive Waste Management, 1891-1896, American Nuclear Society, Las Vegas, Nevada, May 22-26, 1994. 
Diment, G.A., and K.K. Watson, "Stability analysis of water movement in unsaturated porous materials: 3: Experimental studies", Water Resources Research, 21 (7), 979-984, 1985.

Eaton, R.R., N.E. Bixler, and R.J. Glass, "Predicting flow through low-permeability, partially saturated, fractured rock - A review of modeling and experimental efforts at Yucca Mountain", Hydrogeology of Low Permeability Environments, Selected Papers in Hydrogeology, Vol. 2, 28th International Geological Congress, S.P. Neuman, I. Neretnieks editors, Washington, D.C., 239-268, 1990, (SNL paper SAND88-2626C).

Evans, D.D., and T.J. Nicholson, "Flow and transport through unsaturated rock: An overview", Flow and Transport Through Unsaturated Fractured Rock, D.D. Evans and T.J. Nicholson, editors, Geophysical Monograph 42, American Geophysical Union, Washington, DC, 1-10, 1987.

Foltz, S.D., V.C. Tidwell, R.J. Glass, and S.R. Sobolik, "Investigation of fracture matrix interaction: Preliminary experiments in a simple system", Proceedings of the Fourth Annual International Conference on High Level Radioactive Waste Management, 328-335, American Nuclear Society, Las Vegas, Nevada, April 26-30, 1993 (SNL paper SAND922670C).

Glass, R.J., "Laboratory research program to aid in developing and testing the validity of conceptual models for flow and transport through unsaturated porous media", Proceedings of the GEOVAL-90 Symposium on Validation of Geosphere Flow and Transport Models, 275-283, Stockholm, Sweden, May 14-17, 1990, (SNL paper SAND89-2359C).

Glass, R.J., "Modeling gravity driven fingering in rough-walled fractures using modified percolation theory", Proceedings of the 4th International Conference of High Level Radioactive Waste Management, 2042-2053, American Nuclear Society, Las Vegas, Nevada, April 26-30, 1993, (SNL paper SAND92-2792C).

Glass, R.J., and M.J. Nicholl, "Quantitative visualization of entrapped phase dissolution within a horizontal flowing fracture", Geophysical Research Letters, 22(11), 1413-16, 1995. (SNL paper SAND94-3277J).

Glass, R.J. and M.J. Nicholl, "Physics of gravity uriven fingering of immiscible fluids within porous media: An overview of current understanding and selected complicating factors", Geoderma, in press, 1996 (SNL paper SAND95-1278J).

Glass, R.J. and D.L. Norton, "Wetted-region structure in horizontal unsaturated fractures: Water entry through the surrounding porous matrix", Proceedings of the 3rd International Conference on High Level RadiocativeWaste Management, 717-726, American Nuclear Society, Las Vegas, Nevada, April 12-16, 1992, (SNL paper SAND91-2030C).

Glass, R.J., T.S. Steenhuis, and J-Y. Parlange, "Mechanism for finger persistence in homogeneous unsaturated porous media: Theory and verification", Soil Science, 148 (1), 60-70, 1989.

Glass, R.J., and V.C. Tidwell, "Research program to develop and validate conceptual models for flow and transport through unsaturated, fractured rock", Proceedings of the Second Annual International Conference on High Level Radioactive Waste Management, 977-987, American Nuclear Society, Las Vegas, Nevada, April 28-May 3, 1991 (SNL paper SAND90-3042C). 
Glass, R.J., V.C. Tidwell, A.L. Flint, W. Peplinski, and Y. Castro, "Fracture-Matrix interaction in Topopah Spring tuff: Experiment and numerical analysis", Proceedings of the Fifth Annual International Conference on High Level Radioactive Waste Management, 1905-1914, American Nuclear Society, Las Vegas, Nevada, May 22-26, 1994 (SNL paper SAND94-0443C).

Glass, R.J., M.J. Nicholl, and V.C. Tidwell, "Challenging models for flow in unsaturated, fractured rock through exploration of small scale flow processes", Geophysical Research Letters, 22(11), 1457-60, 1995 (SNL paper SAND94-3276J).

Hakami, E., "Water flow in single rock joints", SKB Technical Report 89-08, Swedish Nuclear Fuel and Waste Management Co., 1989.

Ho, C.K., "Assessing alternative conceptual models of fracture flow," Proceedings of the TOUGH Workshop '95, Lawrence Berkeley Laboratory, Berkeley, California, LBL37200 CONF-9503110, 1995.

Kazemi, H. and J.R. Gilman, "Multiphase flow in fractured petroleum reservoirs", in Flow and Contaminant Transport in Fractured Rock, J. Bear, C.F. Tsang, and G. de Marsily, editors, Academic Press, San Diego, CA, 267-323, 1993.

Kwicklis, E.M. and R.W. Healy, "Numerical investigation of steady liquid water flow in a variably saturated fracture network", Water Resources Research, 29 (12), 4091-4102, 1993.

Liu, B., J. Fabryka-Martin, A. Wolfsberg, B. Robinson, and P. Sharma, "Significance of apparent discrepancies in water ages derived from atmospheric radionuclides at Yucca Mountain", Nevada, LANL Report LA-UR-95-572, Los Alamos National Laboratory, 1995.

Martinez, M.J., "Capillary-driven flow in a fracture located in a porous medium", SAND84-1697, Sandia National Laboratories, 1988.

Mualem, Y., "A new model for predicting the hydraulic conductivity of unsaturated porous media", Water Resources Research, 12(3), 513-522, 1976.

Nitao, J.J., and T.A. Buscheck, "Infiltration of a Liquid Front in an Unsaturated, Fractured Porous Medium", Water Resources Research, 27(8), 2099-2112 (1991).

Nicholl, M.J., R.J. Glass and H.A. Nguyen, "Gravity-driven fingering in unsaturated fractures", Proceedings of the Third International Conference on High Level Radioactive Waste Management, 321-331, American Nuclear Society, Las Vegas, Nevada, April 1216, 1992. (SNL paper SAND91-1985C).

Nicholl, M.J., R.J. Glass, and H.A. Nguyen, "Small-scale behavior of single gravity driven fingers in an initially dry fracture", Proceedings of the Fourth Annual International Conference on High Level Radioactive Waste Management, 2023-2032, American Nuclear Society, Las Vegas, Nevada, April 26-30, 1993a. (SNL paper SAND92-2790C).

Nicholl, M.J., R.J. Glass, and H.A. Nguyen, "Wetting front instability in an initially wet unsaturated fracture", Proceedings of the Fourth Annual International Conference on High Level Radioactive Waste Management, 2061-2070, American Nuclear Society, Las Vegas, Nevada, April 26-30, 1993b. (SNL-paper SAND92-2791C). 
Nicholl, M.J., R.J. Glass, and S.W. Wheatcraft, "Gravity-Driven infiltration instability in initially dry nonhorizontal fractures", Water Resources Research, 30 (9), 2533-2546. (SNL paper SAND93-1270J).

Nicholl, M.J. and R.J. Glass, "Wetting phase permeability in a partially saturated horizontal fracture", Proceedings of the Fifth Annual International Conference on High Level Radioactive Waste Management, 2007-2019, American Nuclear Society, Las Vegas, Nevada, May 22-26, 1994. (SNL paper SAND93-2774C).

Peters, R.R. and E.A. Klavetter, "A continuum model for water movement in an unsaturated fractured rock mass", Water Resources Research, 24(3), 416-430, 1988.

Pruess, K., "TOUGH2-A general-purpose numerical simulator for multiphase fluid and heat flow" LBL-29400, Lawrence Berkeley Laboratory, Berkeley, CA, 1991.

Pruess, K. and J.S.Y. Wang, "Numerical modeling of isothermal and nonisothermal flow in unsaturated fractured rock - A review, Flow and Transport Through Unsaturated Fractured Rock, D.D. Evans and T.J. Nicholson, editors, Geophysical Monograph 42, American Geophysical Union, Washington, DC, 11-22, 1987.

Pruess, K., and Y.W. Tsang, "On two-phase relative permeability and capillary pressure of rough-walled rock fractures", Water Resources Research, 26(9), 1915-26, September, 1990.

Russell, C.E., J.W. Hess, and S.W. Tyler, "Hydrogeologic investigation of flow in fractured tuffs, Rainier Mesa, Nevada Test Site", in Flow and Transport Through Unsaturated Fractured Rock, D.D. Evans and T.J. Nicholson, editors, Geophysical Monograph 42, American Geophysical Union, Washington DC, 43-50, 1987.

Saffman, P.G., and G. I. Taylor, "The penetration of a fluid into a porous medium or Hele-Shaw cell containing a more viscous liquid", Proceedings, Royal Society of London, A245, 312-331, 1958.

Snow, D.T., "The frequency and apertures of fractures in rock", Int. J. Rock Mech. Min. Sci., 7, 23-40, 1970.

Thamir, F., E.M. Kwicklis, and S. Anderton, "Laboratory study of water infiltration into a block of welded tuff", Proceedings of the Fourth Annual Intermational Conference on High Level Radioactive Waste Management, 2071-2080, American Nuclear Society, Las Vegas, Nevada, April 26-30, 1993.

Thoma, S.G., D.P. Gallegos, and D.M. Smith, "Impact of fracture coatings on fracture/matrix flow interactions in unsaturated, porous media", Water Resources Research, 28 (5), 1357-1367, 1992.

Tidwell, V.C., "Laboratory investigation of constitutive property upscaling in volcanic tuffs", SAND95-1888, Sandia National Laboratories, Albuquerque, NM, 1995.

Tidwell, V.C., R.J. Glass, and W.J Peplinski, "Laboratory investigation of matrix imbibition from a flowing fracture", Geophysical Research Letters, 22(11),1405-1408, 1995. 
Wang, J.S.Y., and T.N. Narasimhan, "Hydrologic mechanisms governing fluid flow in a partially saturated, fractured, porous medium", Water Resources Research, 21(12), 1861$1874,1985$.

Warren, J.E. and P.J. Root, "The behavior of naturally fractured reservoirs", Society of Petroteum Engineers Journal, 3 (5), 245-255, 1963.

Wilkinsen, D., and J.F. Willemsen, "Invasion percolation: A new form of percolation theory", Journal of Physics A (Mathematical and General), 16 (14), 3365-3376, 1983.

Zimmerman, R.W., G.S. Bodvarsson, and E.M. Kwicklis, "Absorption of water into porous blocks of various shapes and sizes", Water Resources Research, 26(11), 27972806, 1990.

Zimmerman, R.W. and G.S. Bodvarsson, "Effective block size for imbibition or absorption in dual-porosity media", Geophysical Research Letters, 22(11), 1461-1464, 1995.

Zyvoloski, G., B.A. Robinson, Z. V. Dash, and L.L. Trease, "Users manual for the FEHMN application", LA-UR-94-3788, Rev. 1, Los Alamos National Laboratory, Los Alamos, NM, 1995.

\title{
DISCLAIMER
}

\begin{abstract}
This report was prepared as an account of work sponsored by an agency of the United States Government. Neither the United States Government nor any agency thereof, nor any of their employees, makes any warranty, express or implied, or assumes any legal liability or responsibility for the accuracy, completeness, or usefulness of any information, apparatus, product, or process disclosed, or represents that its use would not infringe privately owned rights. Reference herein to any specific commercial product, process, or service by trade name, trademark, manufacturer, or otherwise does not necessarily constitute or imply its endorsement, recommendation, or favoring by the United States Government or any agency thereof. The views and opinions of authors expressed herein do not necessarily state or reflect those of the United States Government or any agency thereof.
\end{abstract}




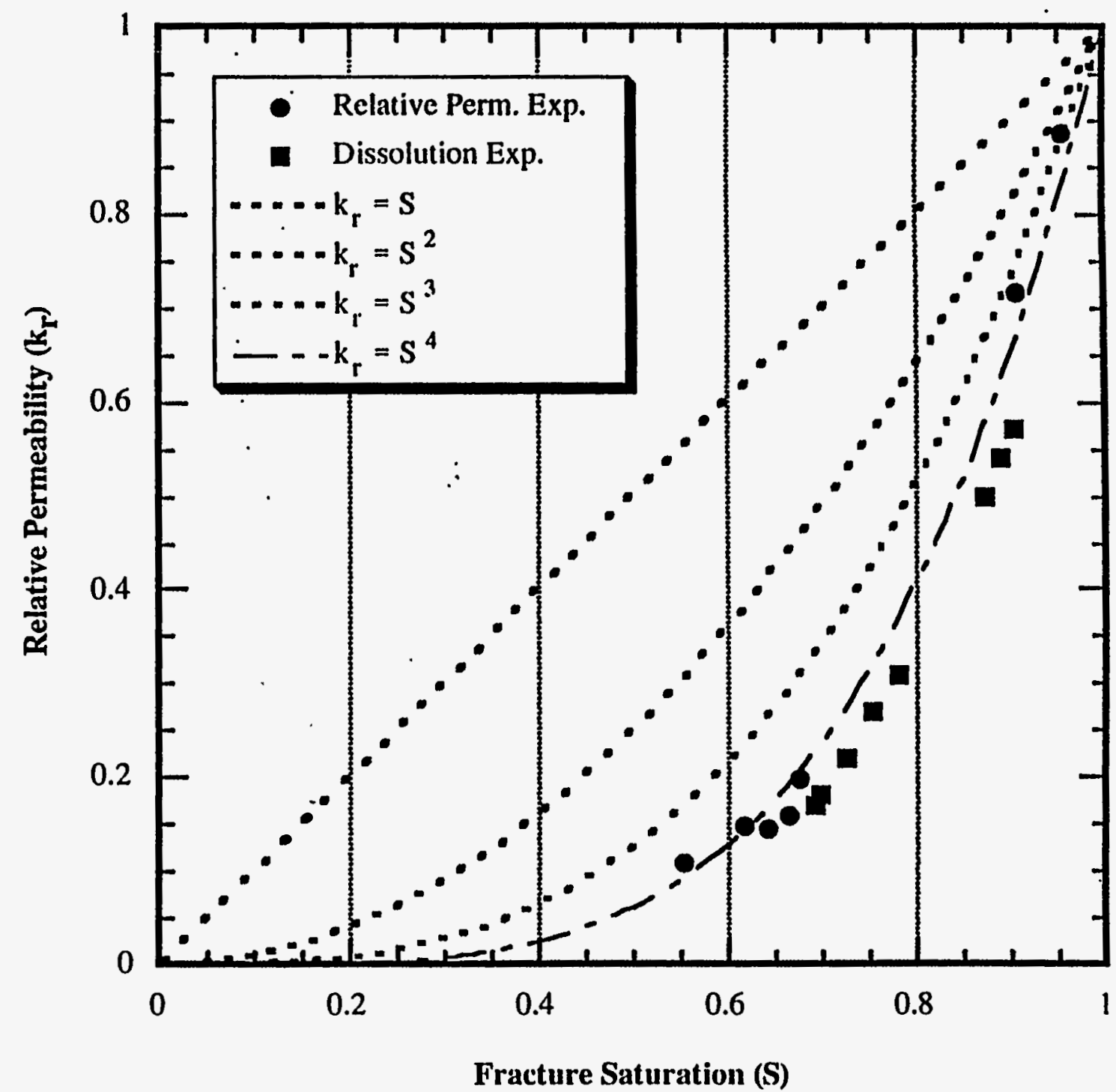

Figure 8: Measured relative permeability in a horizontal analog fracture: In a statistically homogenous (see Figure 3) analog fracture, an initial entrapped phase structure is formed by slow air invasion of the water saturated fracture to breakthrough, followed by re-establishment of water flow. Subsequent flow of deaerated water through the fracture causes the entrapped air phase to be dissolved; resultant effects on fracture relative permeability are shown as red squares (Glass and Nicholl, 1995). Black dots represent experimental data collected from the same fracture for a number of different entrapped phase structures, each with a different phase invasion history (Nicholl and Glass, 1994). Dashed lines illustrate simple power law relationships between saturation (S) and relative permeability ( $\mathrm{k}_{\mathrm{r}}$ ). 


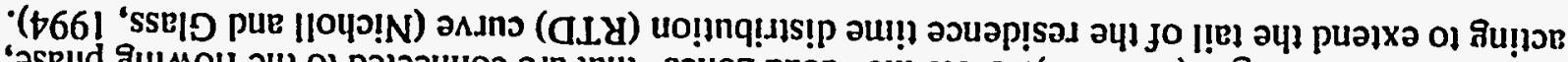

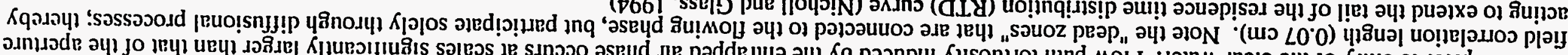

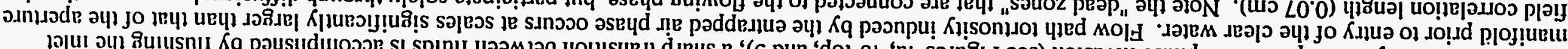

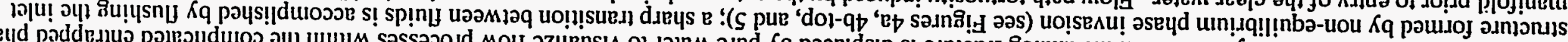

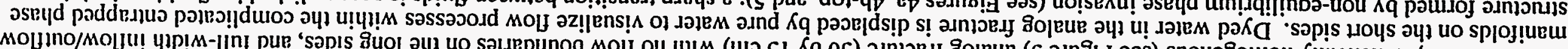

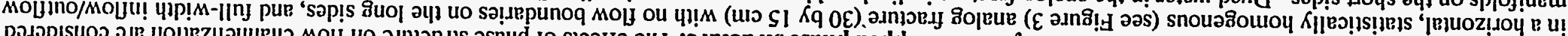

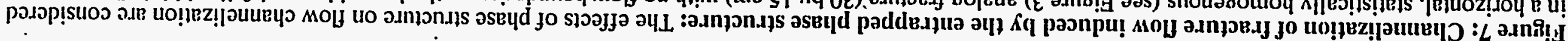

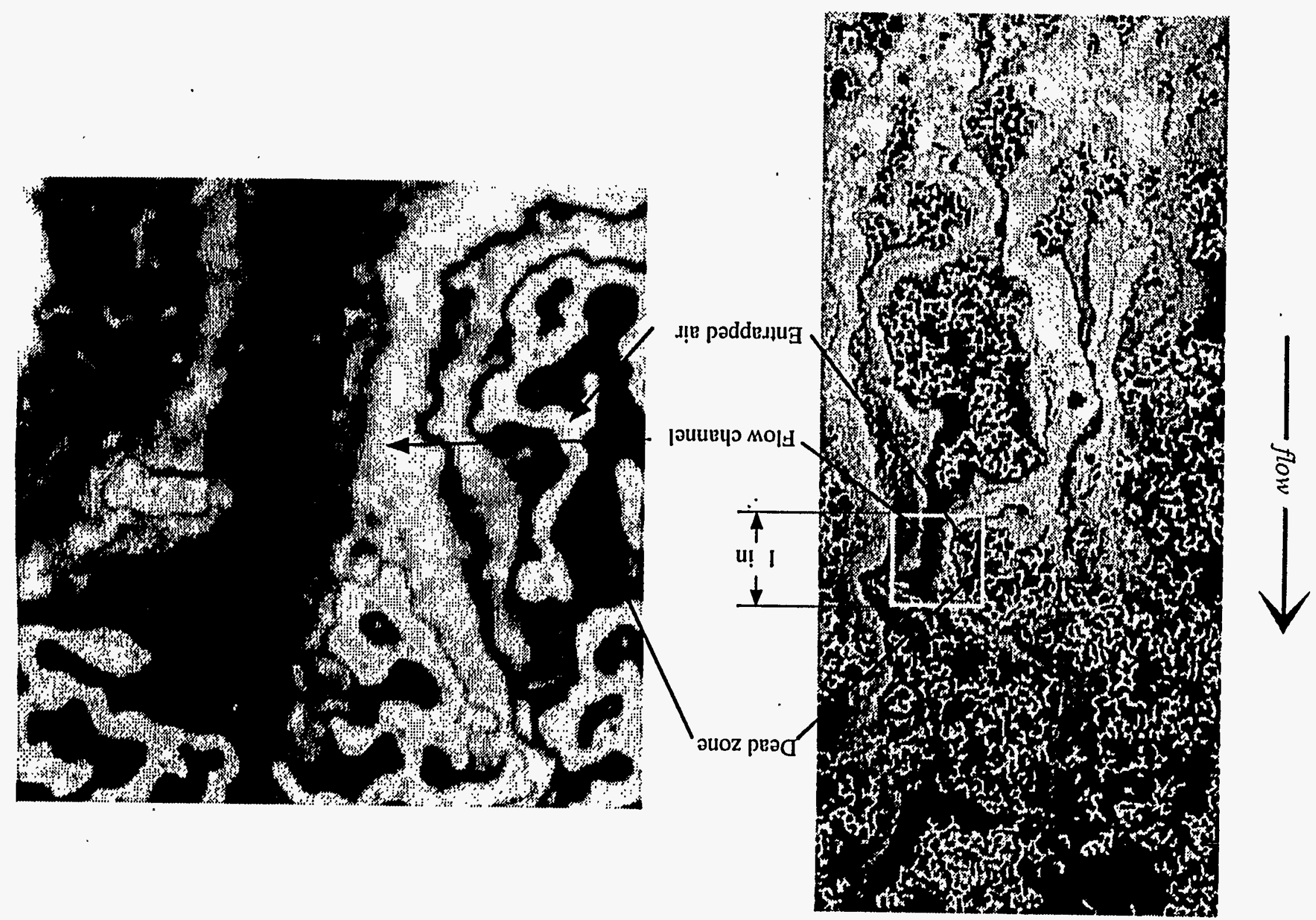




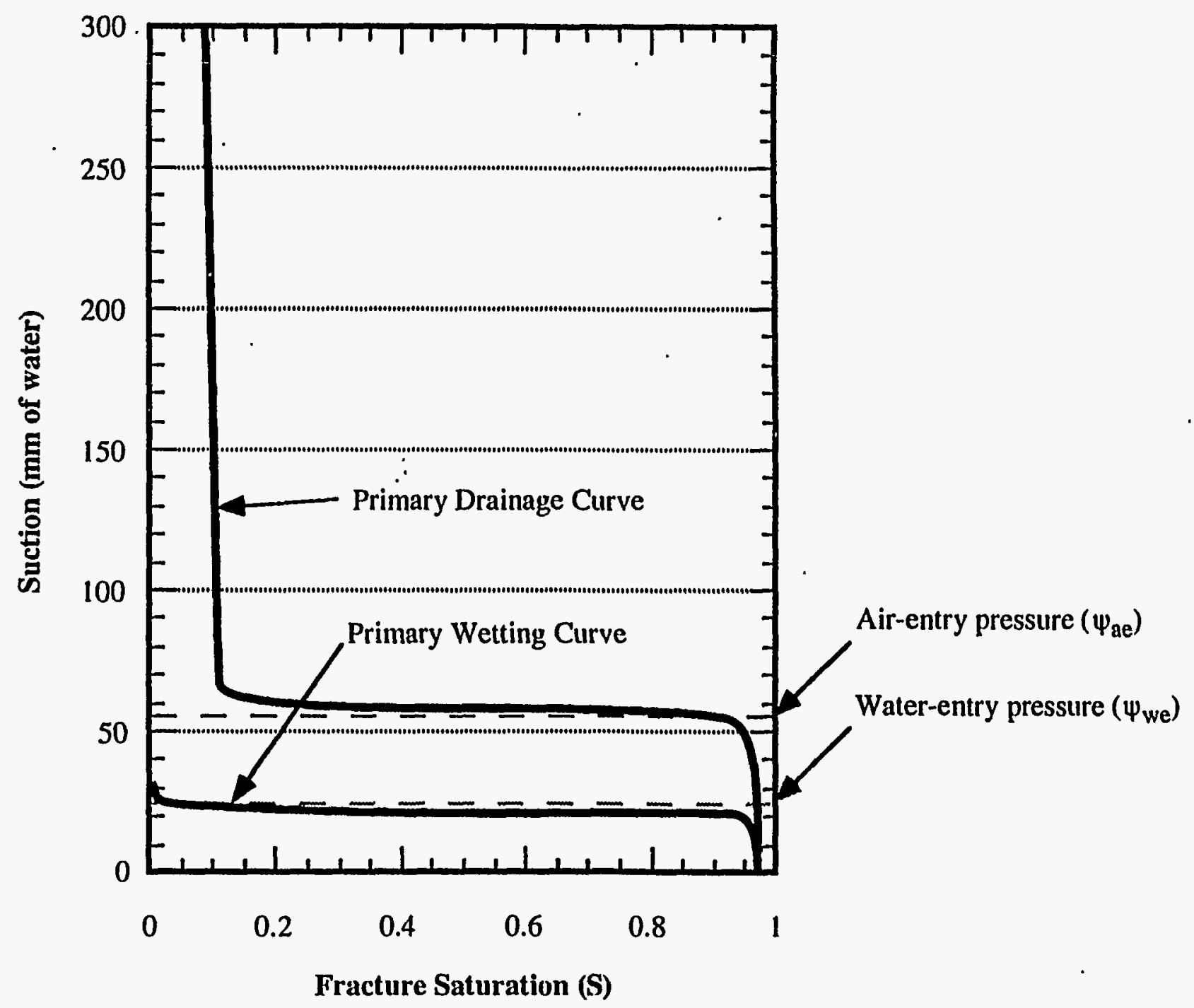

Figure 6b: Hysteresis in fracture pressure-saturation curves: Hysteretic pressure saturation relationships for a statistically homogenous (see Figure 3) analog fracture are inferred from limited data. The vertical capillary rise is assumed to approximate the waterentry pressure $\left(\Psi_{\text {we }}\right)$, while the water height required to sustain free drainage of the vertical fracture is assumed to approximate the airentry pressure $\left(\Psi_{\mathrm{ae}}\right)$. Both values were more than an order of magnitude larger than the aperture correlation length (0.07 $\left.\mathrm{cm}\right)$, implying that the aperture distribution was fully sampled. Residual saturation was estimated by first saturating the fracture, and then allowing free gravitational drainage at a variety of fracture inclinations (Nicholl, Glass, and Nguyen, 1993a). 

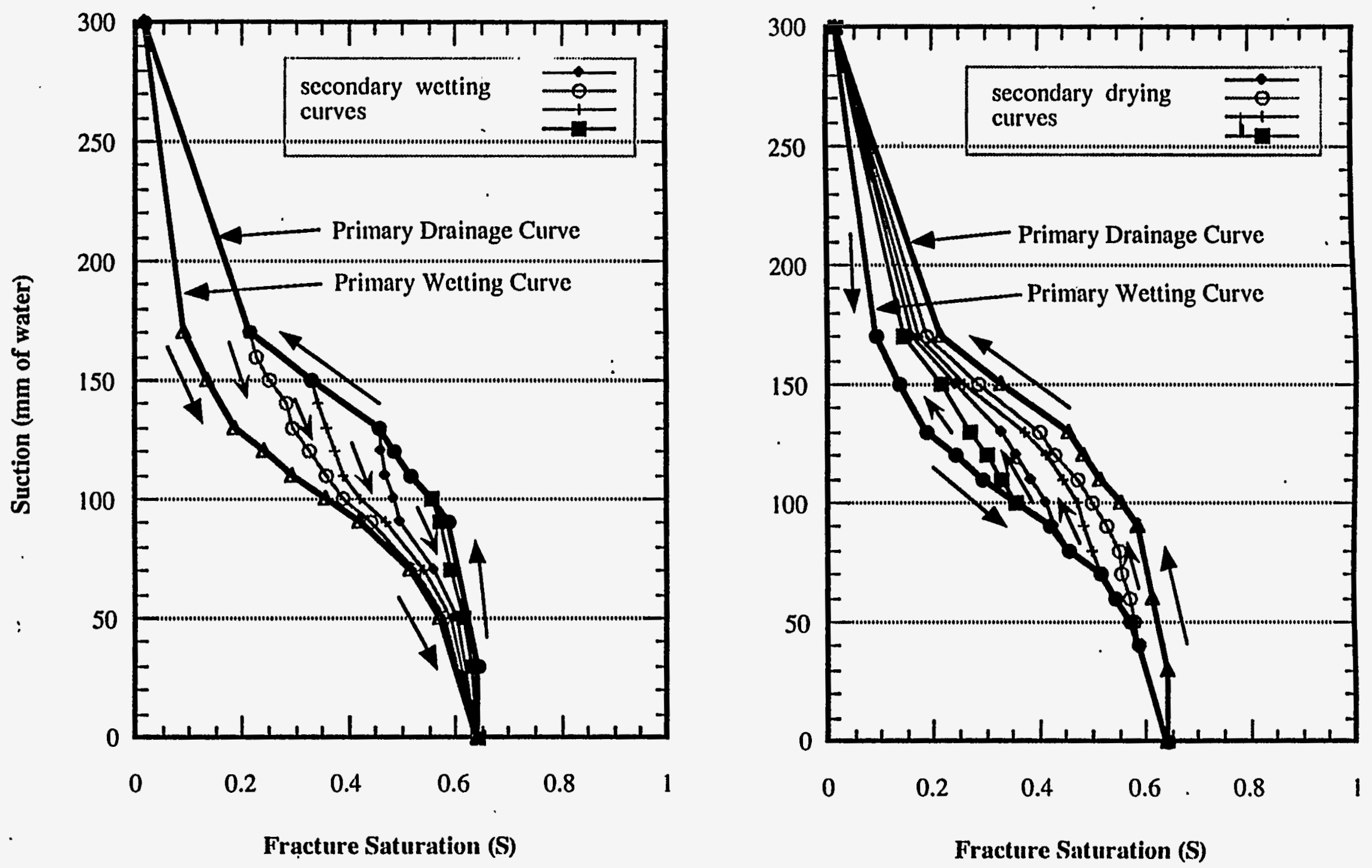

Figure 6a: Hysteresis in fracture pressure-saturation curves for invasion from the matrix: Saturation of a horizontal fracture (15 $\mathrm{x}$ $10 \mathrm{~cm}$ ) as a function of pressure in the adjacent matrix is shown for an analog fracture-matrix system (see Figure 5) (Glass and Norton, 1992). Following measurement of the primary wetting and drainage curves, imposing a sequence of wetting and drainage cycles allows the construction of secondary wetting (left) and drying curves (right). 


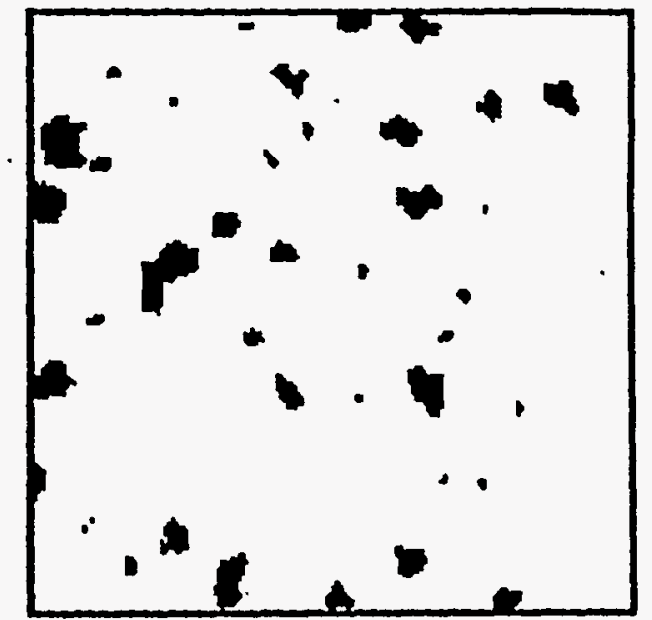

a)

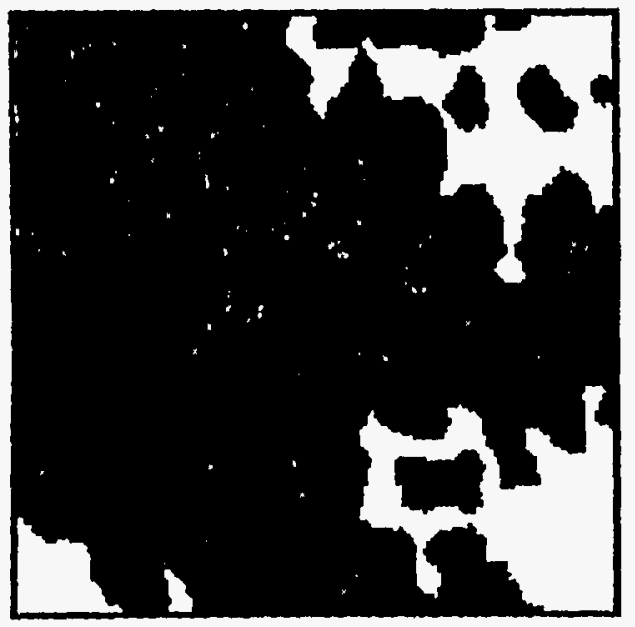

c)

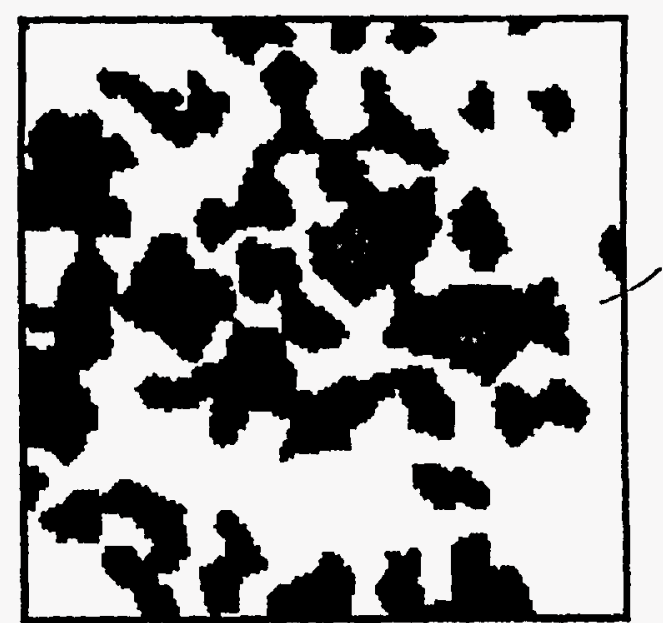

b)
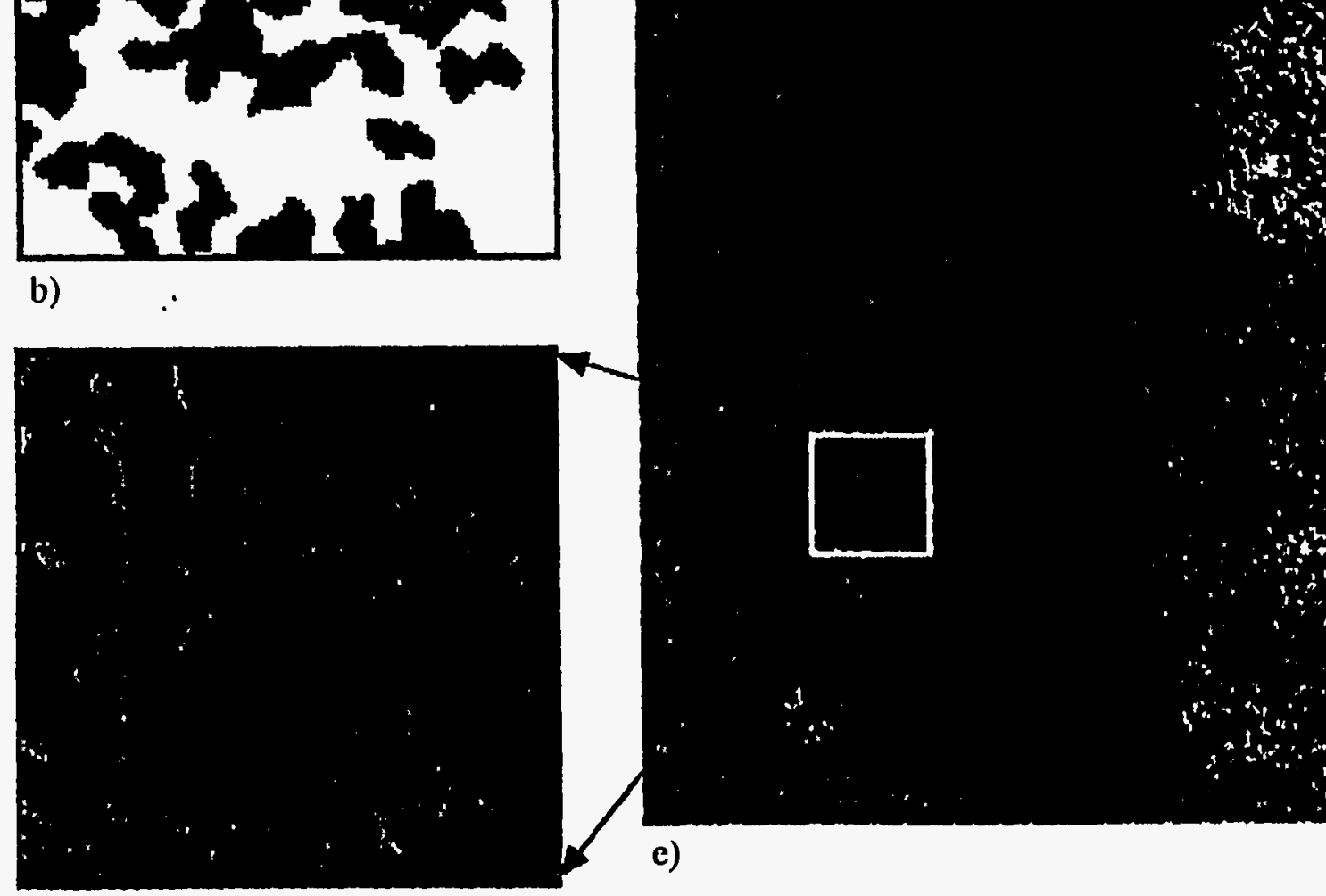

d)

Figure 5: Fracture wetted structure formed by invasion from the matrix: A porous matrix ( $15 \times 10$ ('m) formed from sintered glass beads is held adjacent to a roughened glass platc to form an analog fracture-matrix system. The fracture is oriented along the horizontal plane in order to eliminate gravitational effects on wetted structure formation during wetting from the matrix. The glass plate allows observation of changes in saturation and phase structure that occur in response to variation in matric pressure. The sequence shown here (a-d) illustrates growth of the fracture wetted region (black) in response to increased matric pressure for a $1.5 \times 1.5 \mathrm{~cm}$ portion of the system. Note that water initially enters the fracture at contact points (a); as the wetted regions grow outward from the contact points (b-d) a significant amount of the air phase (white) becomes entrapped (blue). At fracture satiation (d,e) all air in the fracture plane is entrapped; incremental increases in matric pressure will not increase fracture saturation (Glass and Norton, 1992). 


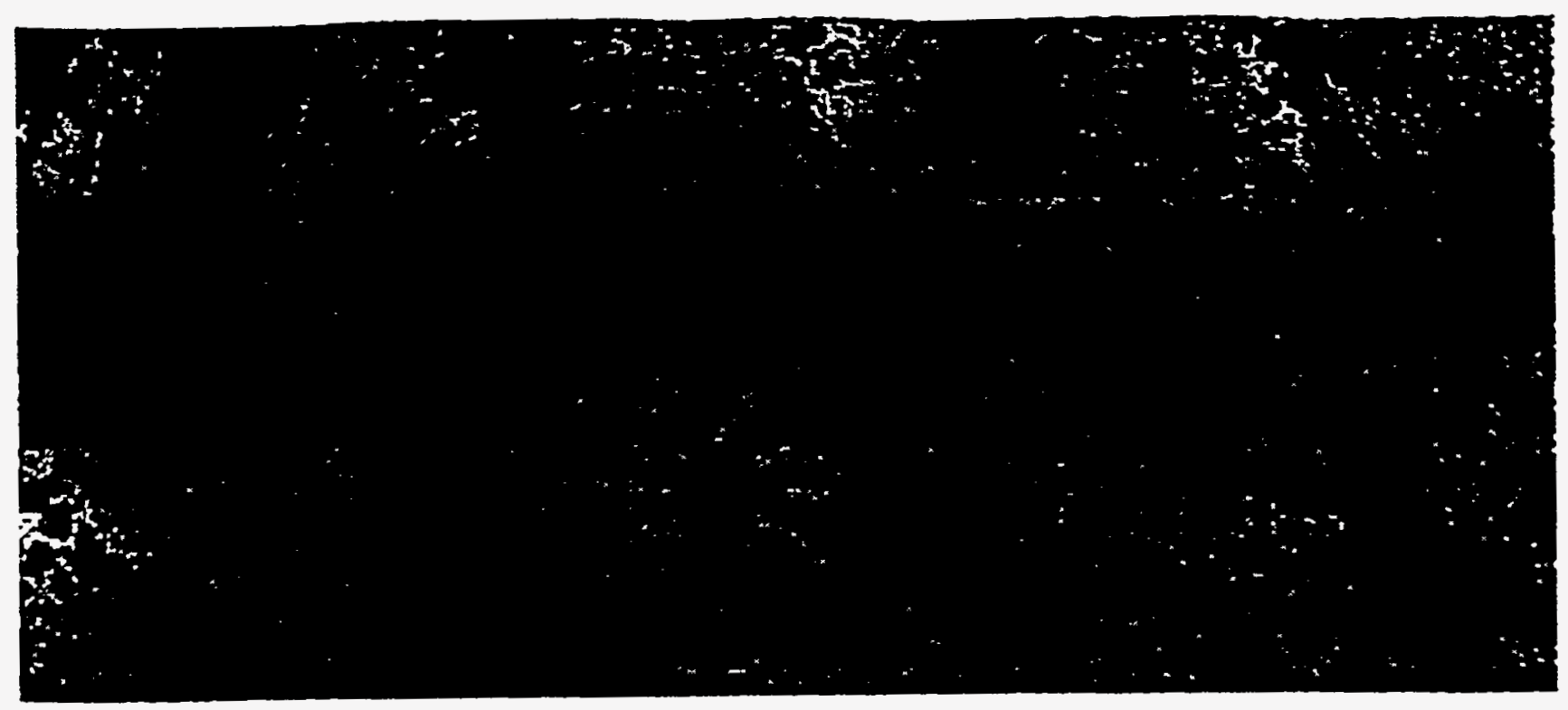

$\left(S_{W}=0.62, k_{r}=0.15\right)$
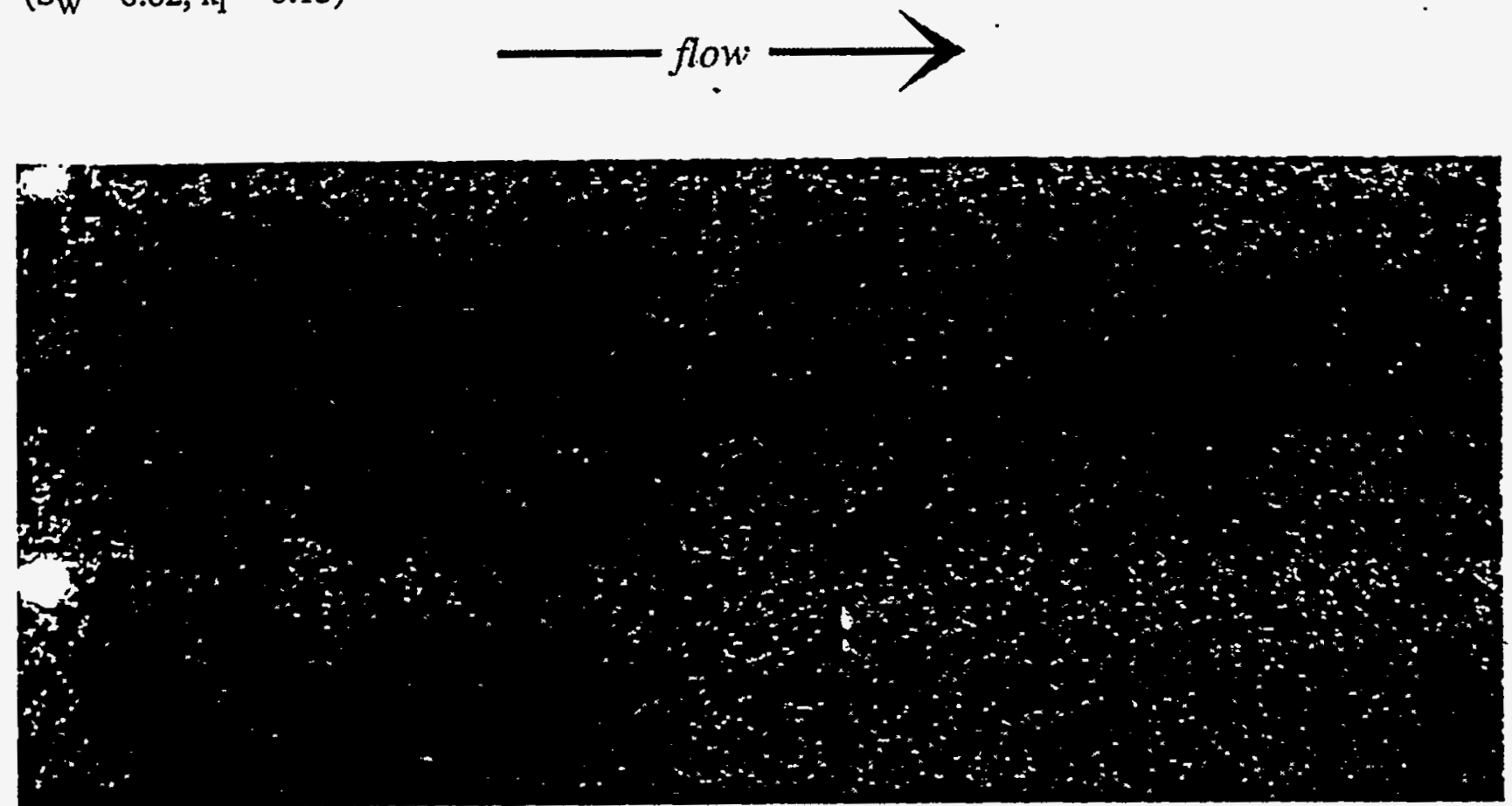

$\left(S_{W}=0.66, k_{I}=0.16\right)$

Figure 4b: Wetted structure formed by invasion from the fracture edge: The effects of phase invasion history on relative permeability are considered in a horizontal, statistically homogenous (see Figure 3) analog fracture ( 30 by $15 \mathrm{~cm}$ ) with no flow boundaries on the long sides and full-width inflow/outflow manifolds on the short sides. Top - Air invasion of the water saturated fracture to breakthrough is followed by steady flow of water (black); resulting in large, complicated, and wellconnected entrapped air structures (blue). Bottom - Drainage of the water saturated fracture to residual through application of suction, followed by steady water flow creates a much more uniform entrapped phase structure. Although phase structures for these two cases differ significantly, water phase saturation ( $S_{\text {) }}$ ) and relative permeability $(\mathrm{k})$ are similar. However, solute transport properties will be radically dffferent; channelization in the fnore complicated structure (top) shortens first arrival time, while dead zones will act to extend the tail of the residence time distribution curve (Nicholl and Glass, 1994). 


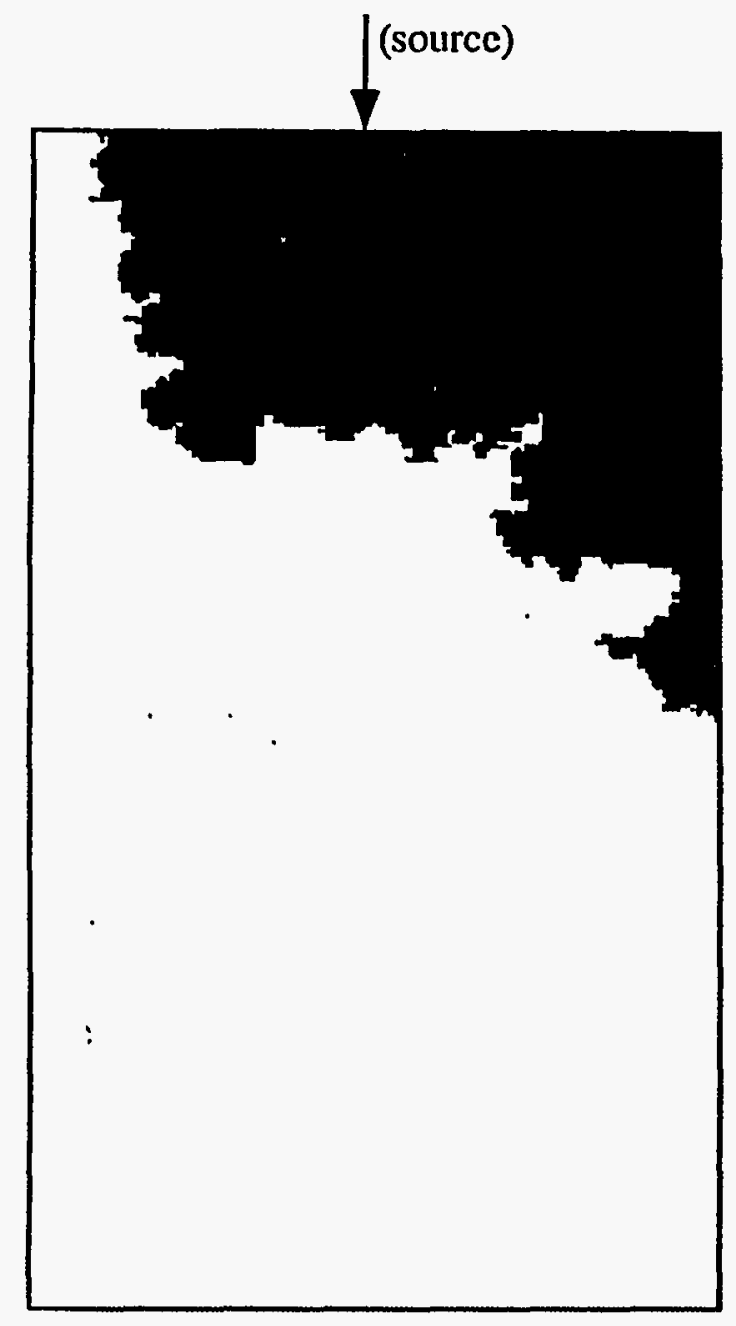

a) $\mathrm{t}=95$ minutes

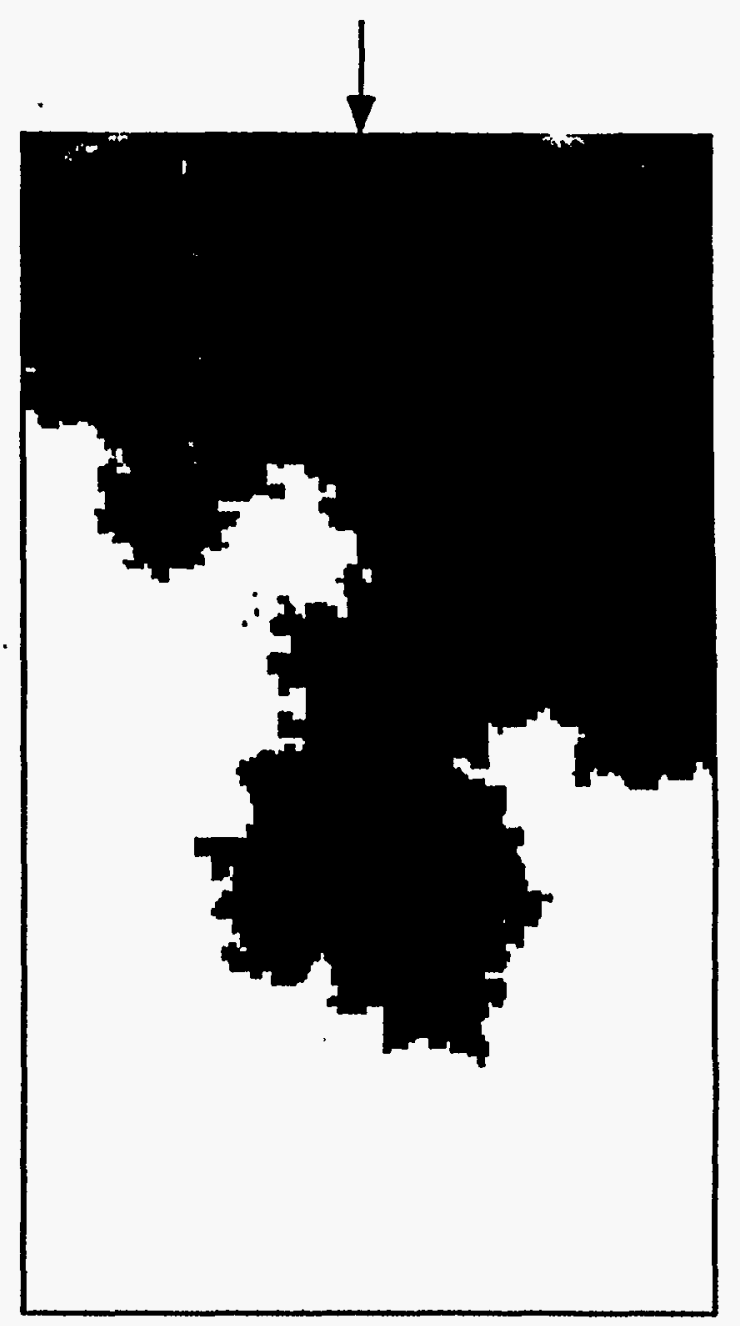

b) $\mathrm{t}=175$ minutes

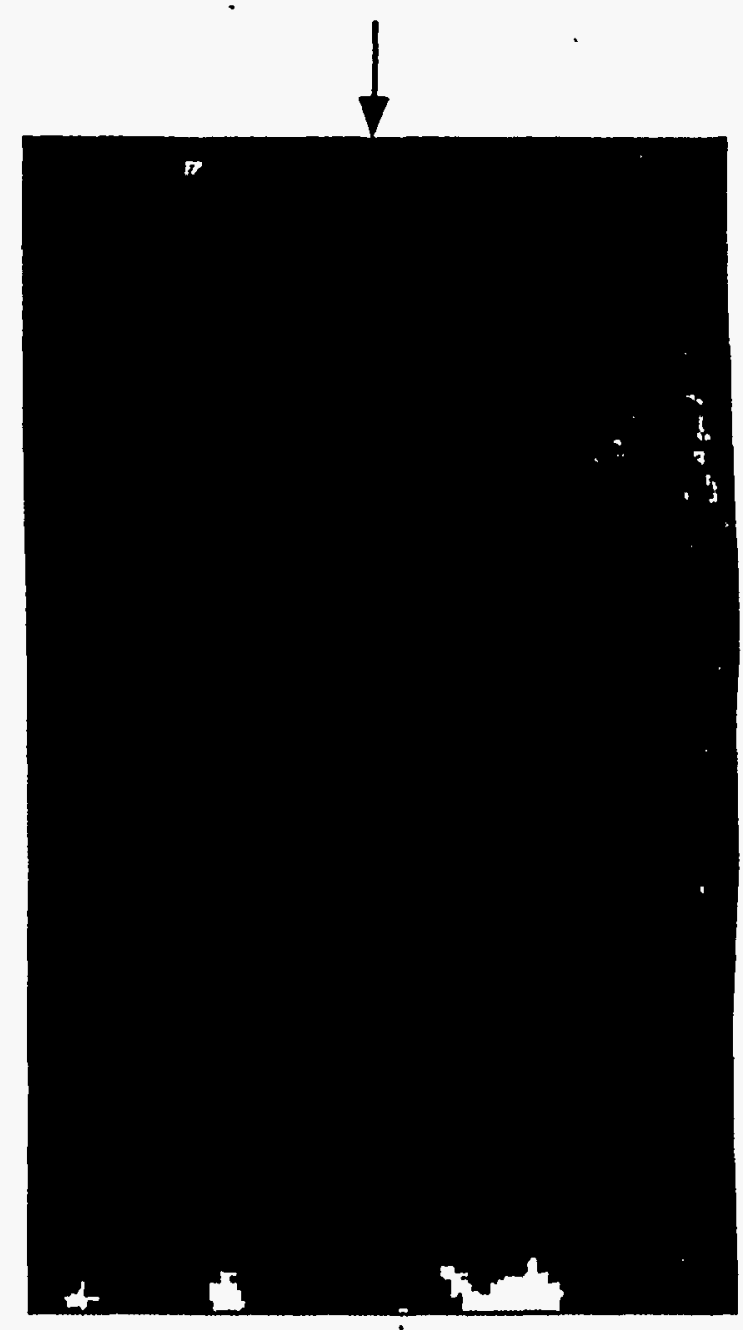

c) $\mathrm{t}=380$ minutes

Figure 4a: Wetted structure formed by invasion from the fracture edge: The slow displacement of air (white) by water (black) is considered in a horizontal, statistically homogenous (see Figure 3$)$, analog fracture (30 by $15 \mathrm{~cm}$ ); all fracture boundaries are held at atmospheric pressure and water is imbibed from a point source on one edge (red arrows). In order to approximate aperture filling under quasi-static conditions, water is imbibed into the fracture under suction at a rate $(1.44 \mathrm{ml} / \mathrm{hr})$ designed to keep viscous forces small with respect to capillary forces. The invading water phase (black) forms features much larger than the correlation length of the aperture ficlu $(0.07 \mathrm{~cm}$ ) and entrapped air structures (blue) at a variety of scales. The invasion process is shown at 95 (a), 175 (b), and 380 (c) minutes after initiation of flow (Glass, 1993). 

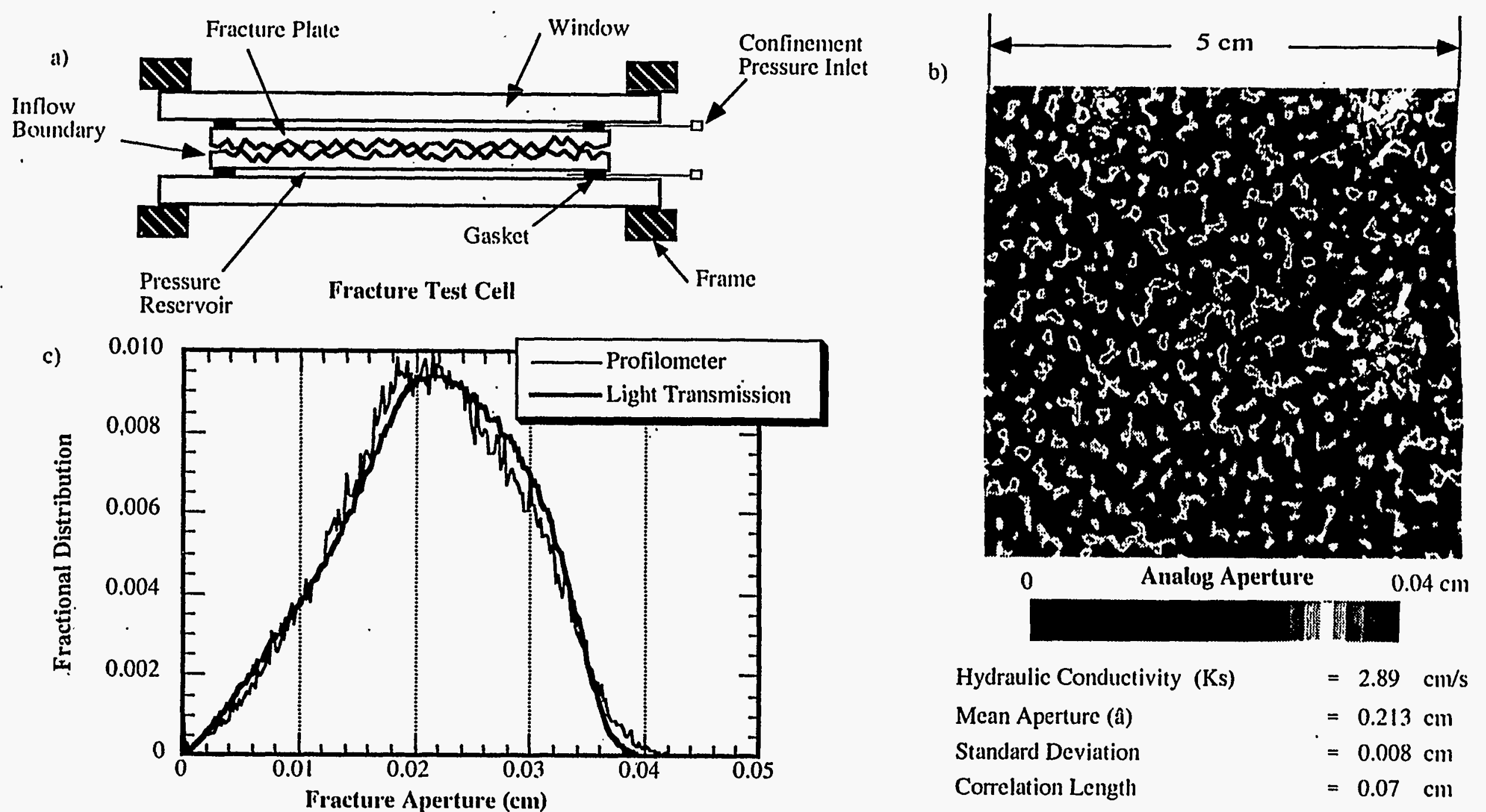
Hydraulic Conductivity (Ks)
Mean Aperture (â)
$=2.89 \mathrm{~cm} / \mathrm{s}$
Standard Deviation
$=0.213 \mathrm{~cm}$
Correlation Length
$=0.008 \mathrm{~cm}$
$=0.07 \mathrm{~cm}$

Figure 3: Fabrication of a reproducible, statistically homogeneous rough-walled analog fracture: A test cell (a) is used to hold the two halves of an analog fracture (size of up to $50 \times 100 \mathrm{~cm}$ ). Data is captured by passing light through the analog fracture and measuring changes in intensity incluced by fluids within the aperture field (air, water, dye, sucrose solutions). Acquisition of data in this manner requires that the analog be transparent or trans licent; appropriate materials include textured glass plates, flat glass plates, and replicas of natural or machined fracture surfaces cast in transparent epony. In order 10 eliminate warpage of the fracture surfaces, and thus assure a repeatable aperture field, a confinement pressure (1ypically 20 psi) is applied nomilil to the fracture plane (see figure for location of pressure reservoirs). A statistically homogenous aperture field is formed by using plates of commercially available textured glass for each half of the fracture. The resultant experimental fracture is orders of magnitude larger in lateral extent than the aperture correlation length and thus is considered statistically homogeneous at the macroscopic scale; a small segment of the aperture ficld is shown for illustrative purposes (b). Measured hydraulic properties of the analog fracture are consistent with those reported for natural fractures $\left(\hat{\mathrm{a}}=0.0084-0.0462 \mathrm{~cm}\right.$ and $\mathrm{K}_{\mathrm{s}}=1.14-14.0$ $\mathrm{cm} / \mathrm{s})$ by Hakami (1989) and the mean apertures $(0.012-0.541 \mathrm{~cm})$ reported by Snow (1970). Aperture measurement requires clear and lyed fluids with index of refraction matched to that of the analog fracture. Lambert's law for light absorption is then applied on a pixel-by-pixel basis to images of the fracture filled with each fluid; the results compare well to measurements (c) based on laser profilometry (Glass, 1993; Nicholl and Glass, 1994). 
a)

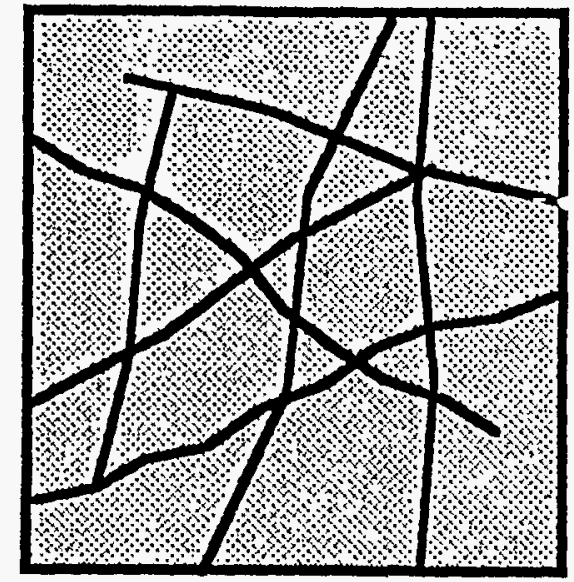

2-D Fractured System

c)

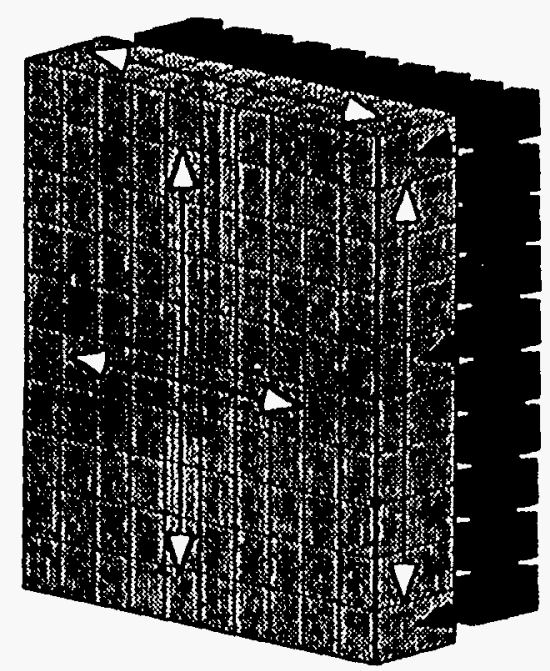

Dual Porosity

\begin{tabular}{ll}
\hline KEY & \\
$\square$ & Matrix Domain \\
$\leftrightarrow \rightarrow$ & Fracture Domain \\
$\leftrightarrow$ & $\begin{array}{l}\text { Interaction within } \\
\text { domains } \\
\text { Interaction between } \\
\text { domains }\end{array}$ \\
\hline
\end{tabular}

Figure 2: Continua models for flow through fractured rock: Conceptual drawings of various continuum models are shown; the systcm is restricted to two-dimensions for clarity. (a) Slab of fractured porous matrix to be modeled. (b) Composite continuum -.each block in the slab represents a composite of fracture and matrix properties. (c) Dual porosity - flow is restricted to the fracture network (purple blocks). The properties of each block in the fracture network are assumed to be continuous. Each fracture block communicates with a matrix block (red); however, no communication occurs between matrix blocks, they act solely as storage for the fracture network. (d) Dual permeability - matrix (red) and fracture (purple) networks are treated as separate continua; a transfer term is used to describe interactions between the two systens. b)

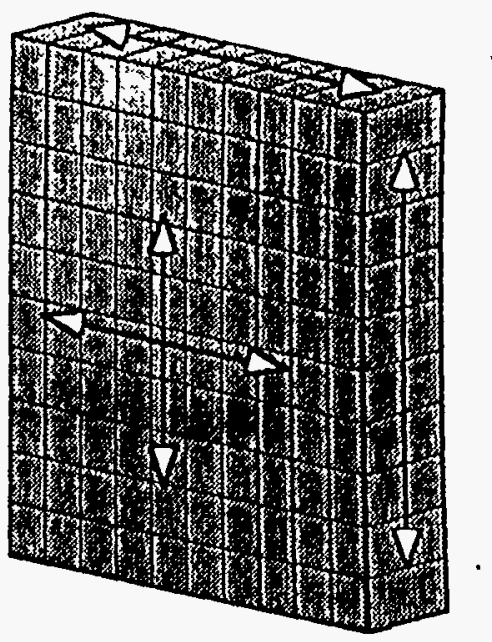

Composite Continuum

d)

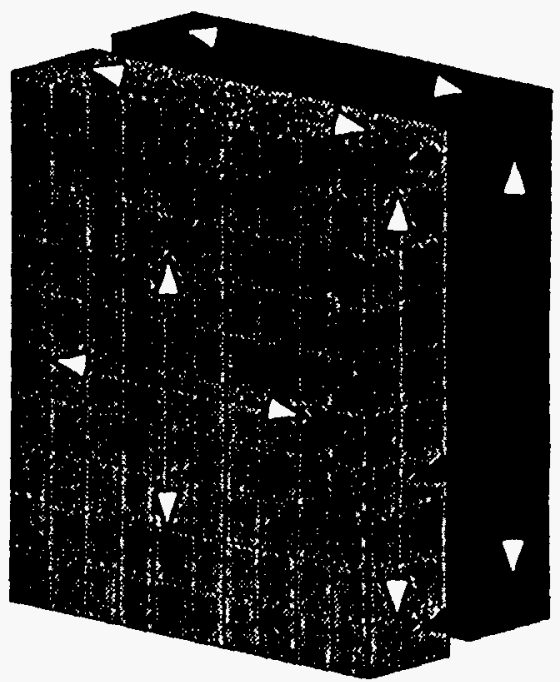

Dual Permeability 
-14th

$\because$ -

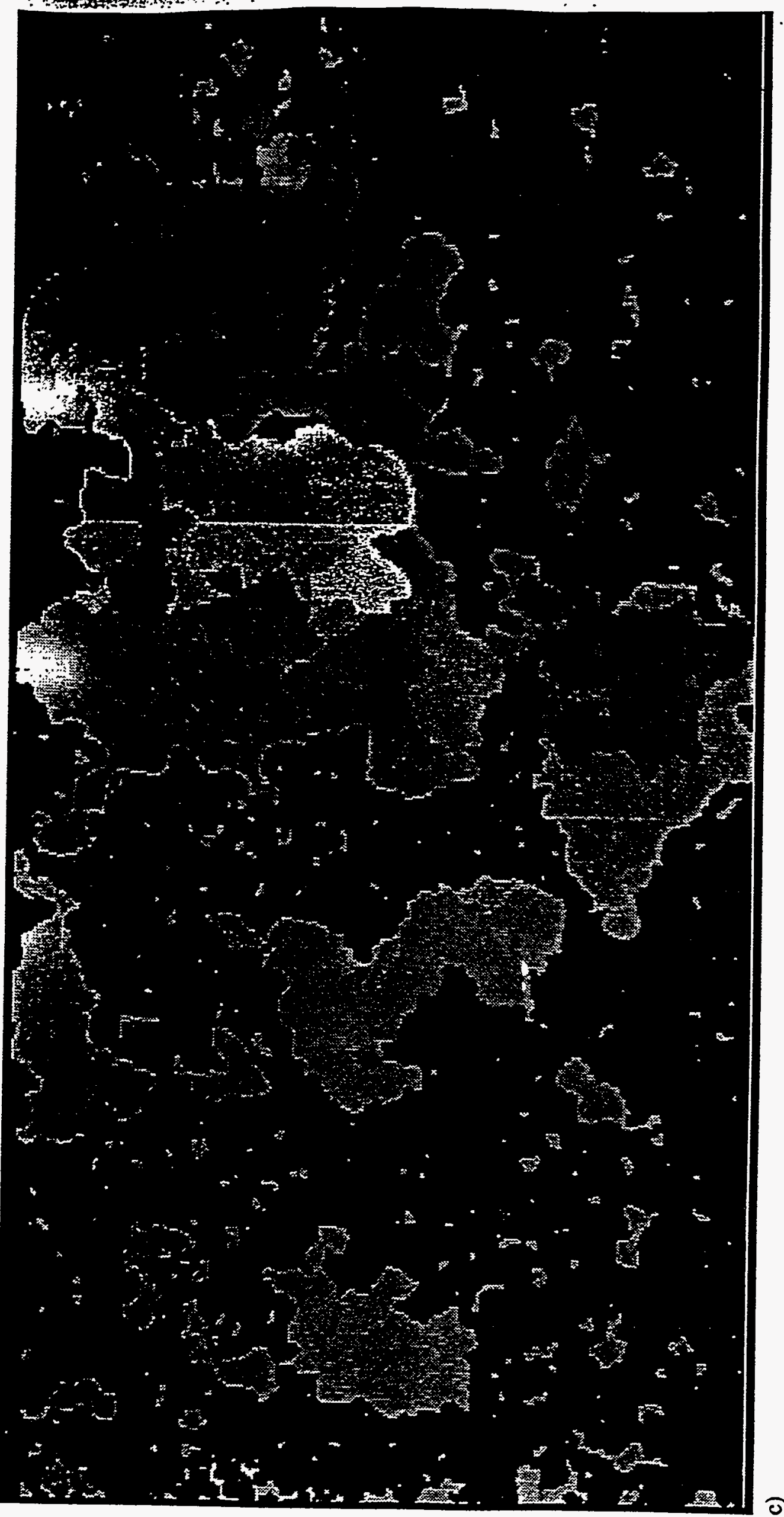




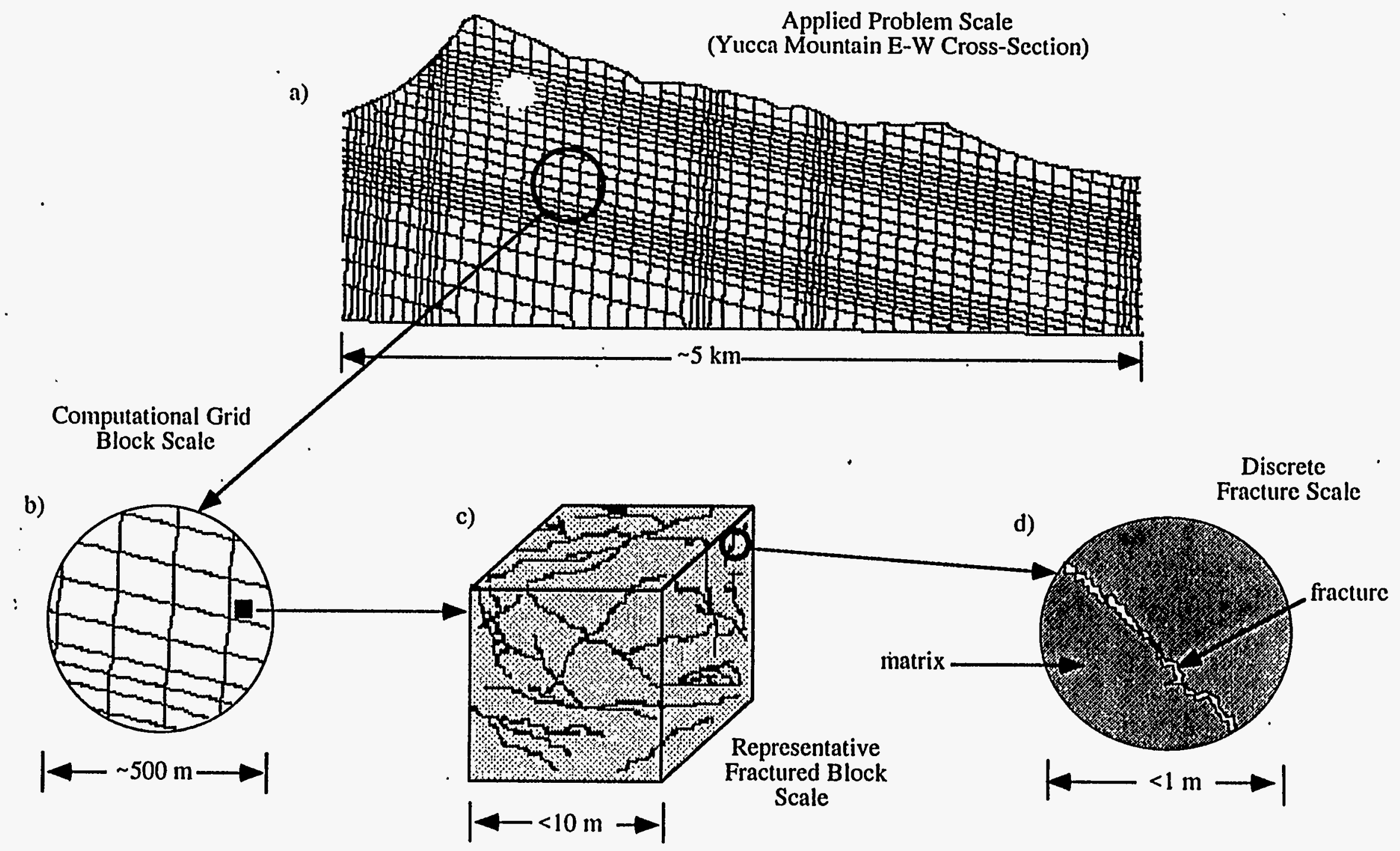

Figure 1: Averaging inherent in the use of equivalent continua models: It is neither possible nor desirable to model large field problems (a) at the scale of individual fractures (d). It is however, essential that numerical models be formulated in a manner that is consistent with the behavior of individual fractures (d) and fracture networks (c). Several discrete scales of averaging for both material properties and physical processes may be required to move from the scale of a single fracture (d) to that of a computational grid block (b). 


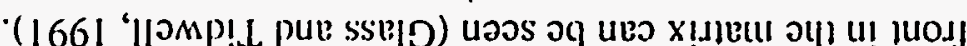

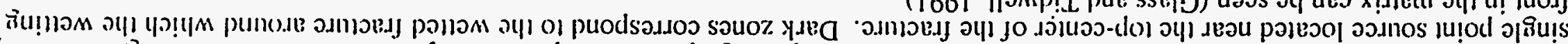

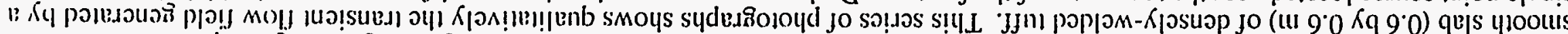

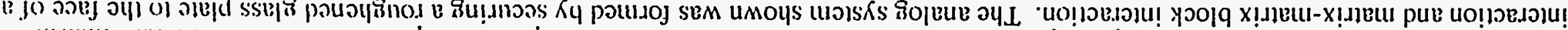

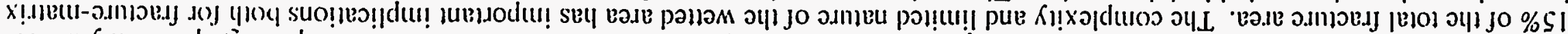

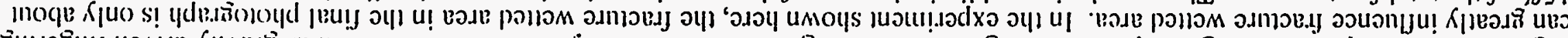
สแ!

$(0$

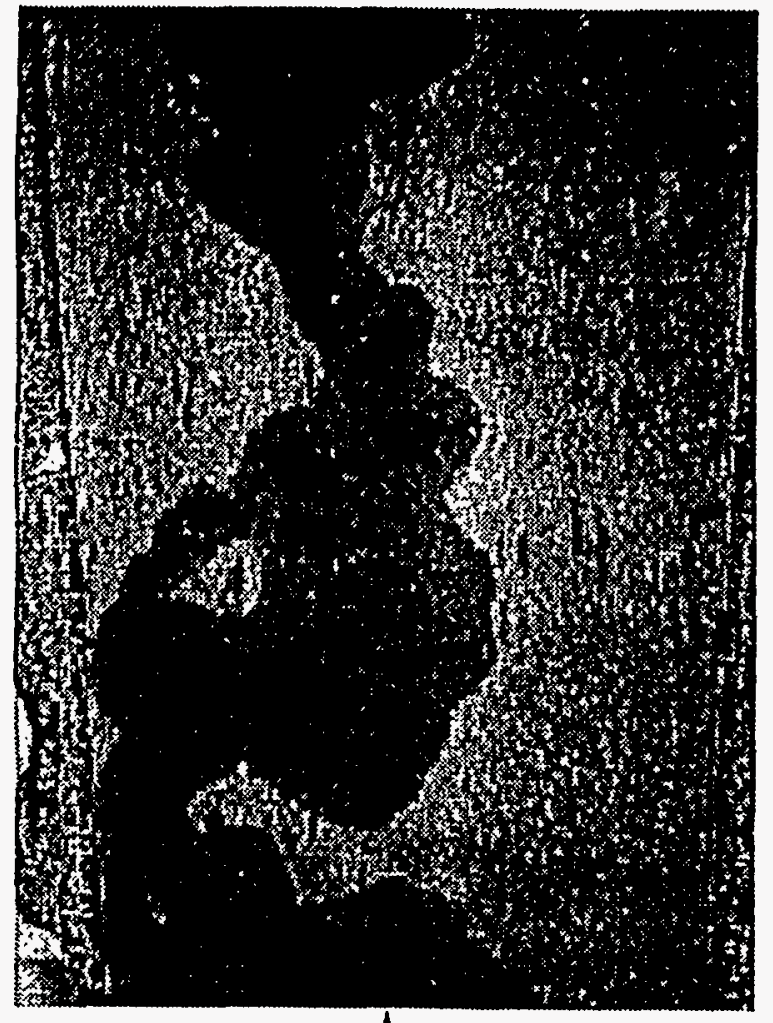

4
$(9$

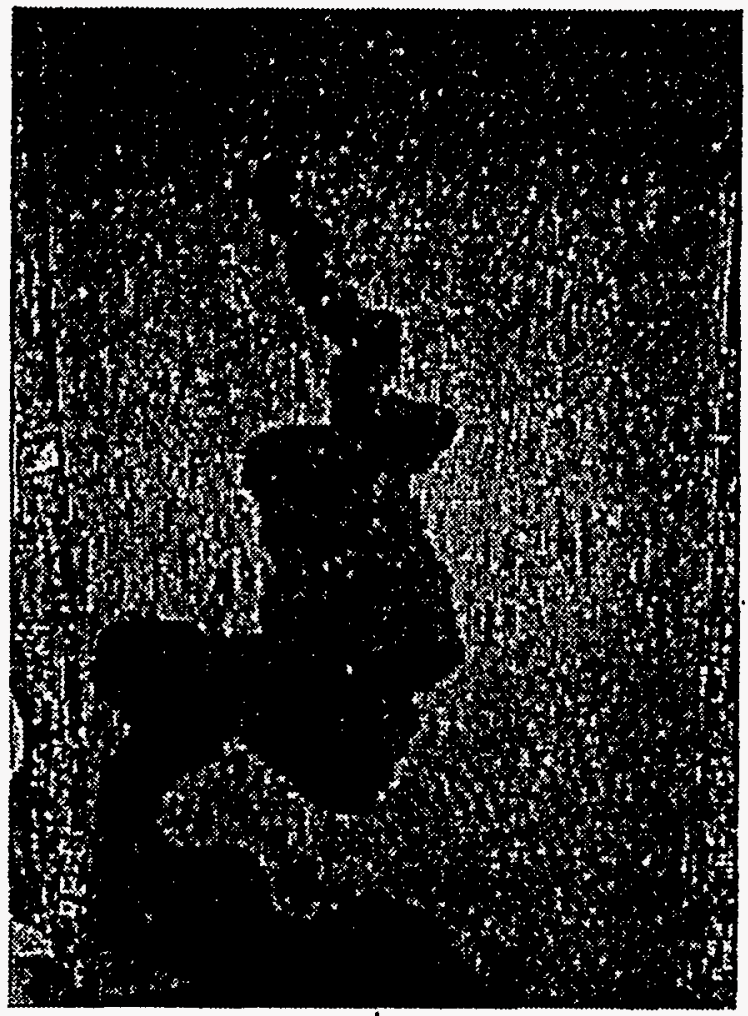

1

$(\mathfrak{e}$

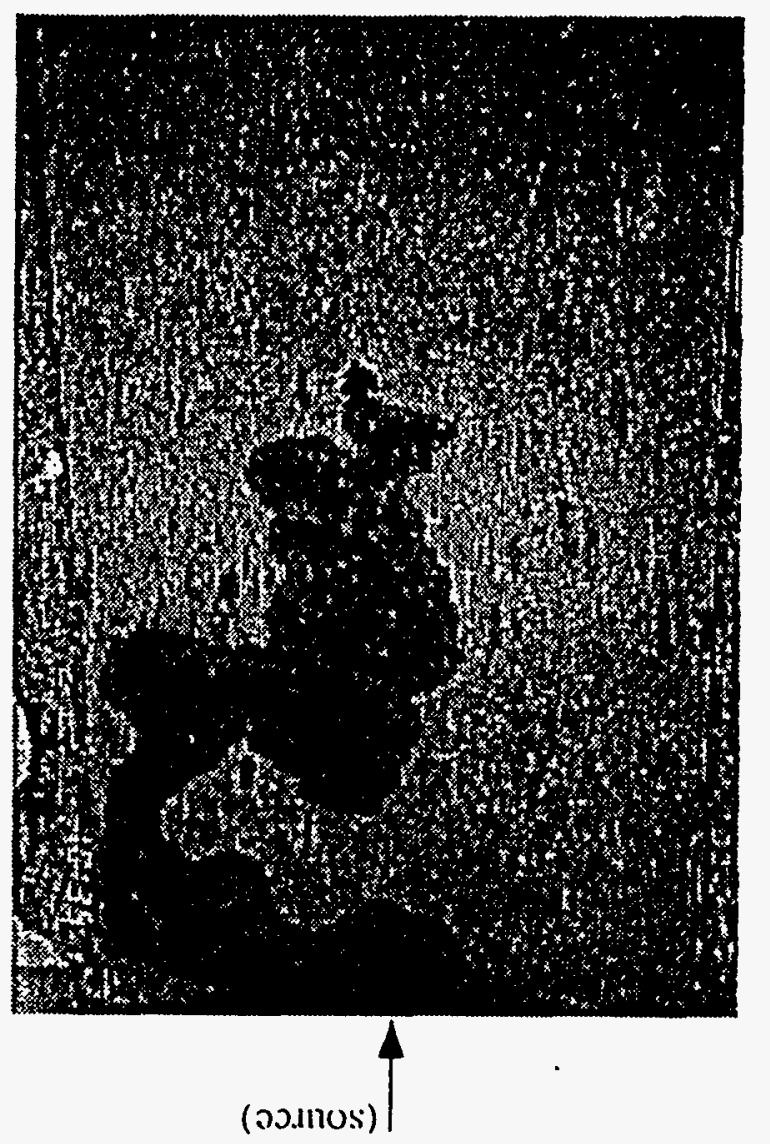




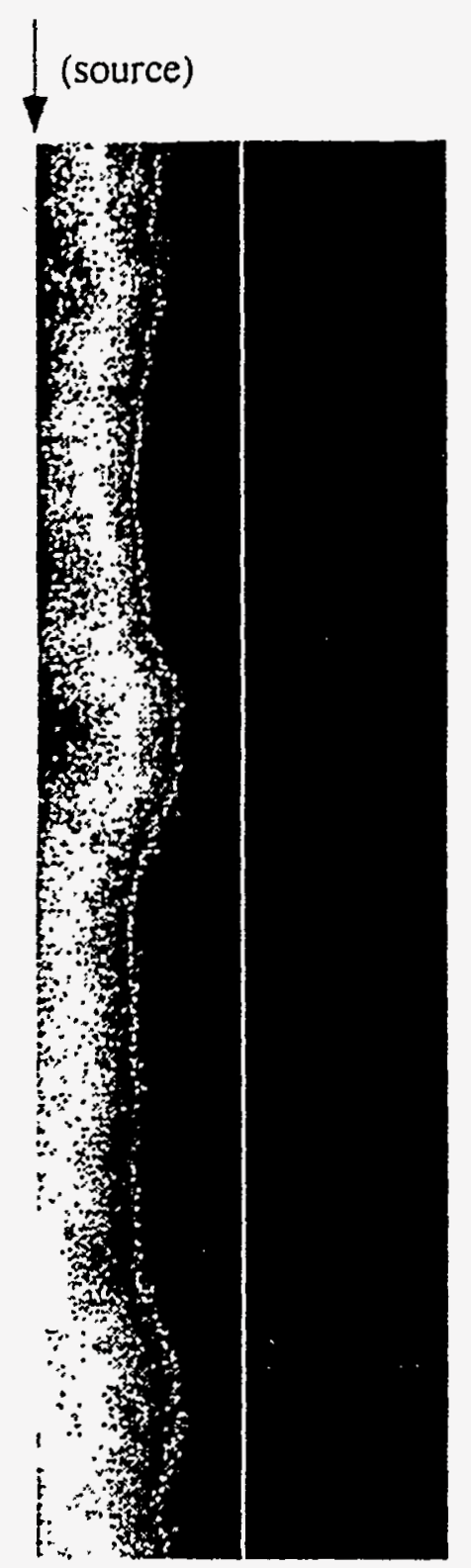

a) $t=115$ minutes

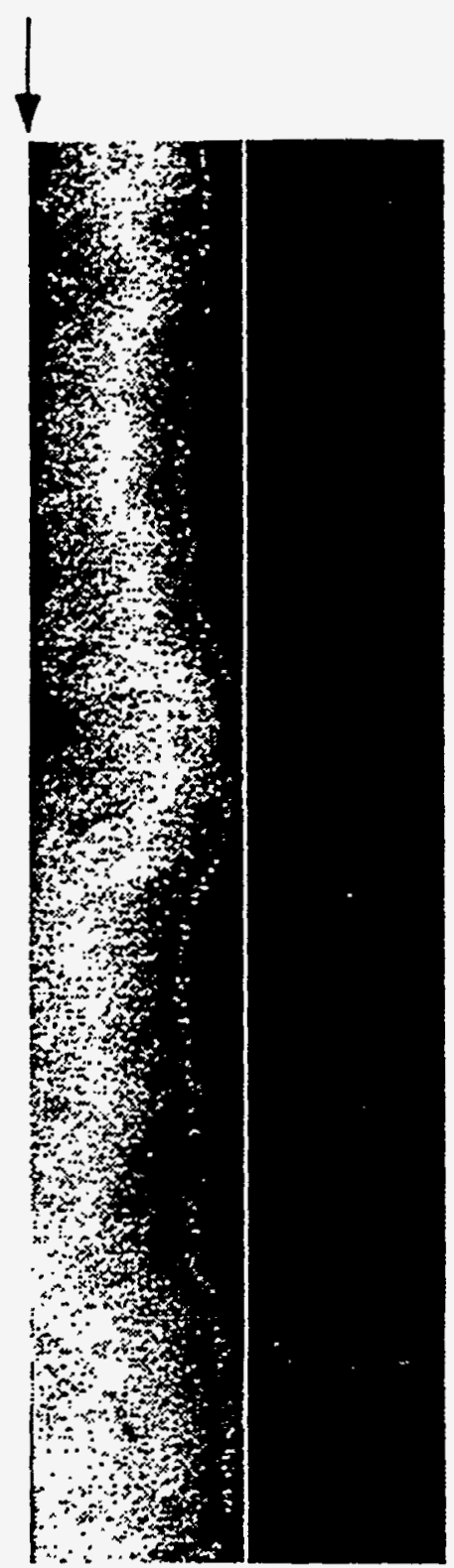

b) $\mathrm{t}=233$ minutes

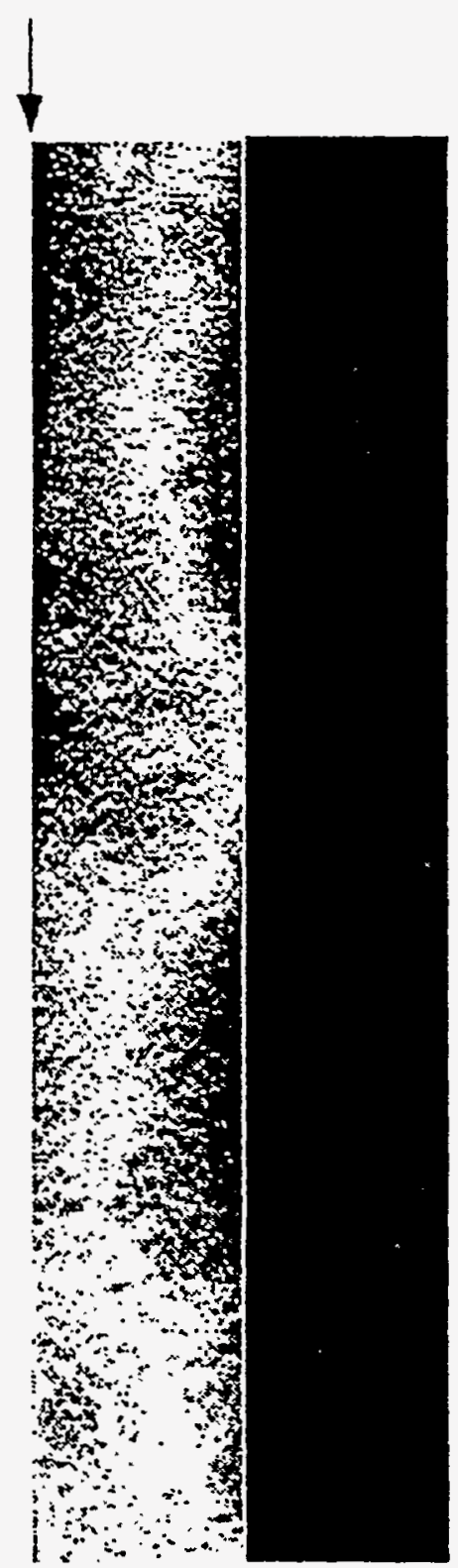

c) $\mathrm{t}=355$ minutes

Figure 42: Capillary barrier formed by a vertical fracture: Regardless of orientation (compare with figure 41), unsaturated fractures have a significant influence on ensemble matrix block flow. The fracture-matrix system shown here is composed of four volcanic tuff slabs (each measuring 50 $\mathrm{cm}$ tall, $7 \mathrm{~cm}$ wide, and $2.54 \mathrm{~cm}$ thick, only the right two slabs are shown) separated by three vertical slot fractures. The central fracture (source) was set to a gap of $0.1 \mathrm{~mm}$ between the slabs while the outer two fractures were set with no air gap (slabs in contact). Water was supplied to the center fracture but not to the outer fractures. Transient flow fields are measured using the $x$-ray' absorption lechnique. Although near saturated conditions are reached near the capillary barrier, water has not spanned the fracture; relative saturation ( 0 to 1 ) is depicted by the standard color bar (black (0)-bluegreen-yellow-red(1)). Images were collected at (a) 115 , (b) 233, and (c) 355 minutes after initiation of flow. 


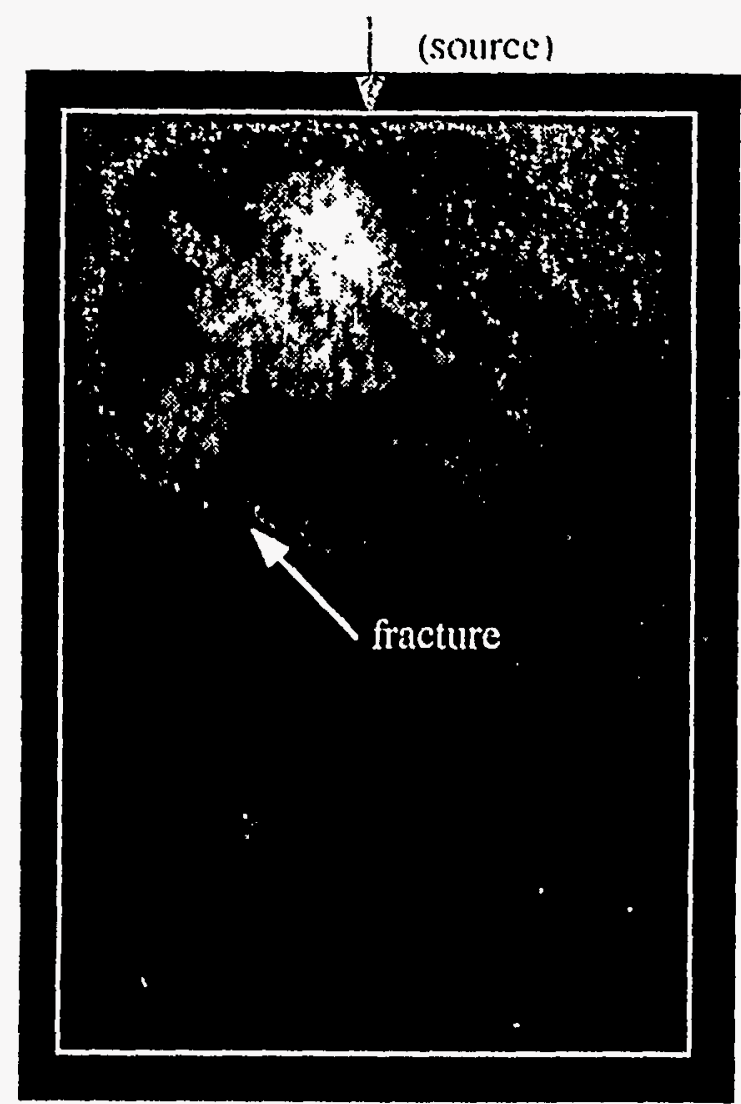

a)

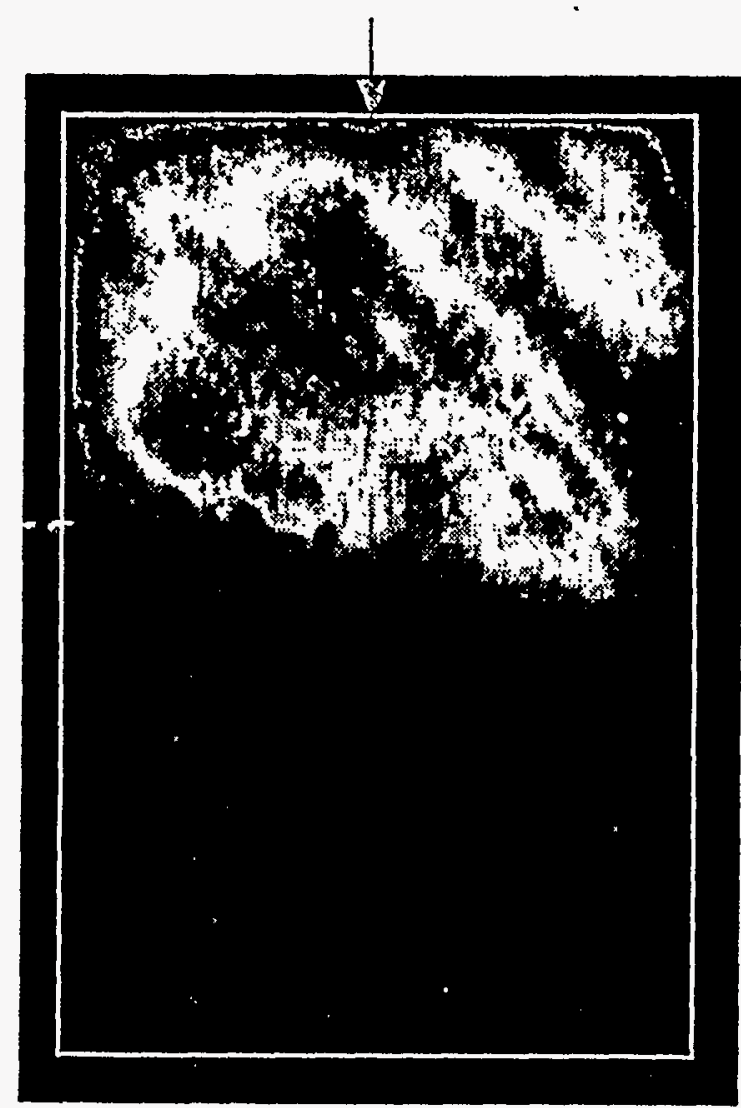

b)

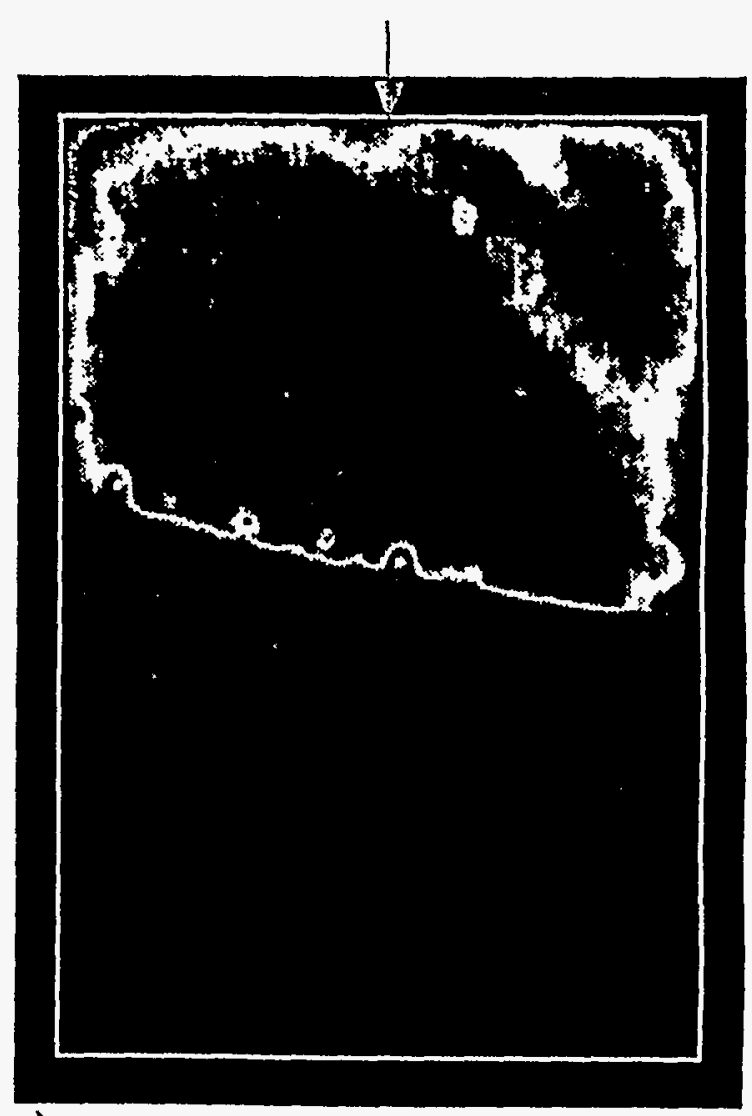

c)

Figure 41: Capillary barrier formed by a near horizontal fracture: Inter-matrix block communication and hence ensemble block permeability can be greatly restricted by the presence of dry fractures as shown here. The fracture-matrix system shown is composed of a sintered glass plate ( 15 by 10 by $0.6 \mathrm{~cm}$, delineated by the white line) broken by a rough walled fracture. Water was supplied to the upper matrix block while light transmission was used to qualitatively image the transient flow fields; relative saturation (0 io 1 ) is depicted by the standard color bar (black (0)-blue-green-yellow-red(1)). In (a) the unsaturated fracture prevents inter-matrix block flow, (b) matrix saturation increases and water breaks across fracture near the left side of the slab, and (d) the upper matrix reaches near saturated conditions, yet minimal water has crossed the fracture (Glass and Tidwell, 1991). 


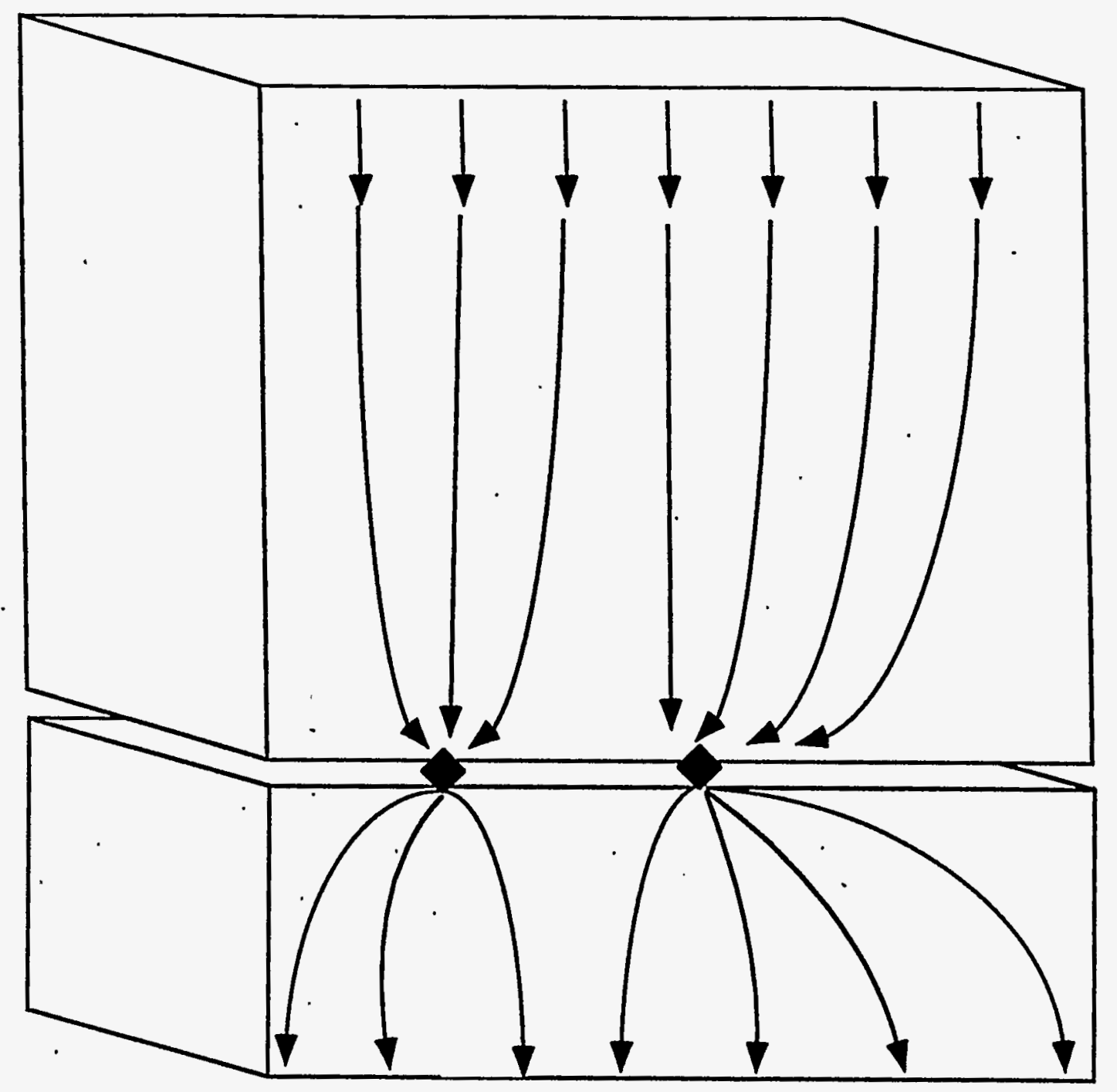

Figure 40: Tortuosity induced by limited inter-block connection: Unsaturated fractures will act as capillary barriers to flow between adjacent matrix blocks; flow will cross the gap either at contact points or water filled apertures. By restricting the area of contact, a macroscopic tortuosity is induced; thereby reducing relative permeability of the matrix block ensemble. 


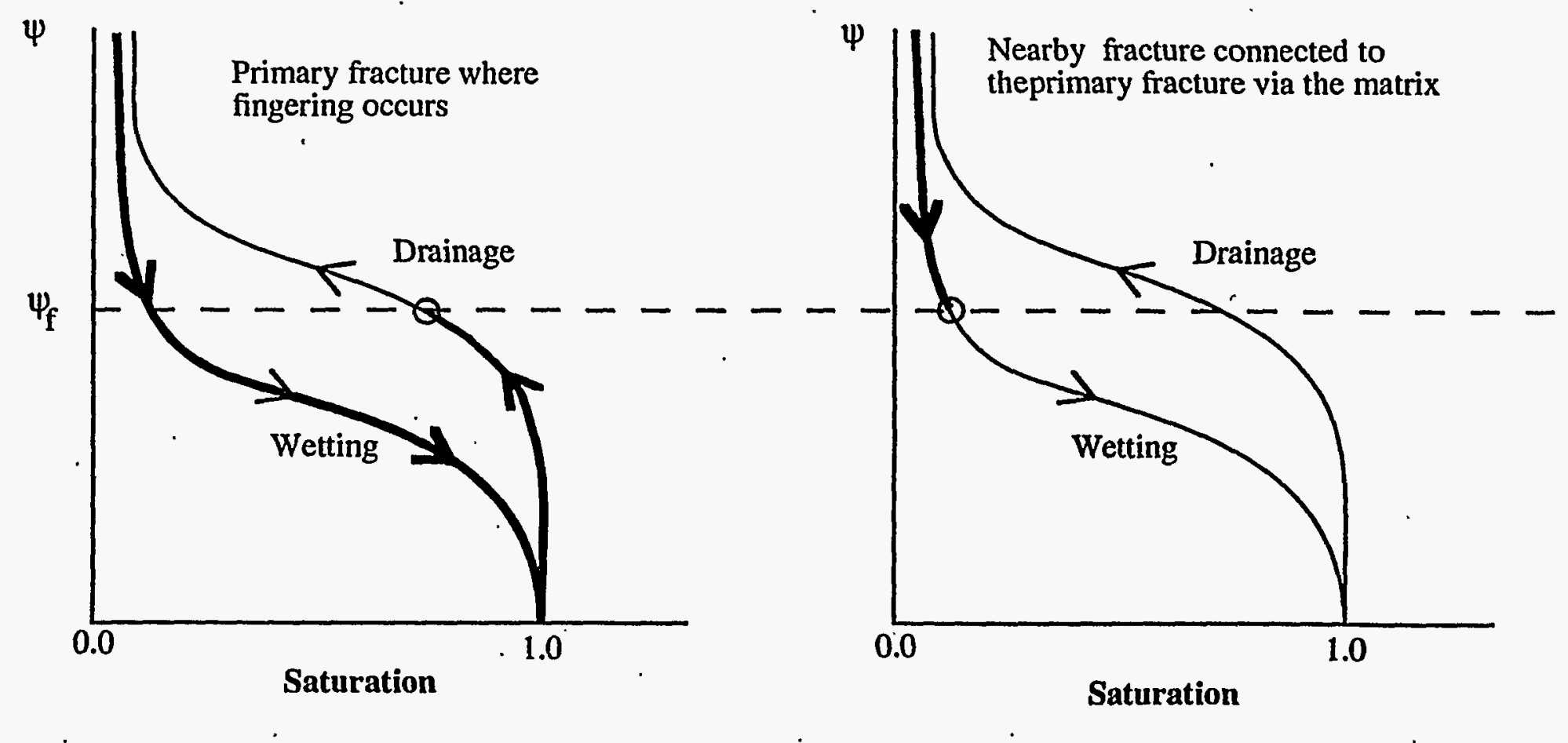

Figure 39: Hysteresis induced inhibition of water transfer between adjacent, vertical fractures: Water transfer from a flowing fracture, through the matrix, and into adjacent non-flowing fractures will be inhibited by hysteresis in the fracture pressure-saturation relationships, even if the fractures are identical. At pressure equilibrium along the horizontal plane, the flowing fracture will be on the drainage curve, while the adjacent non-flowing fractures will be on the wetting curve with correspondingly lower saturations. Nicholl and Glass (1994) reported that small reductions in the saturation of horizontal fractures resulted in significant reduction of the fracture relative permeability. A similar mechanism has been shown to create two-zoned moisture content fields in homogeneous sands after the onset of gravity-driven instability (Glass et al., 1989; Glass and Nicholl, 1996). 


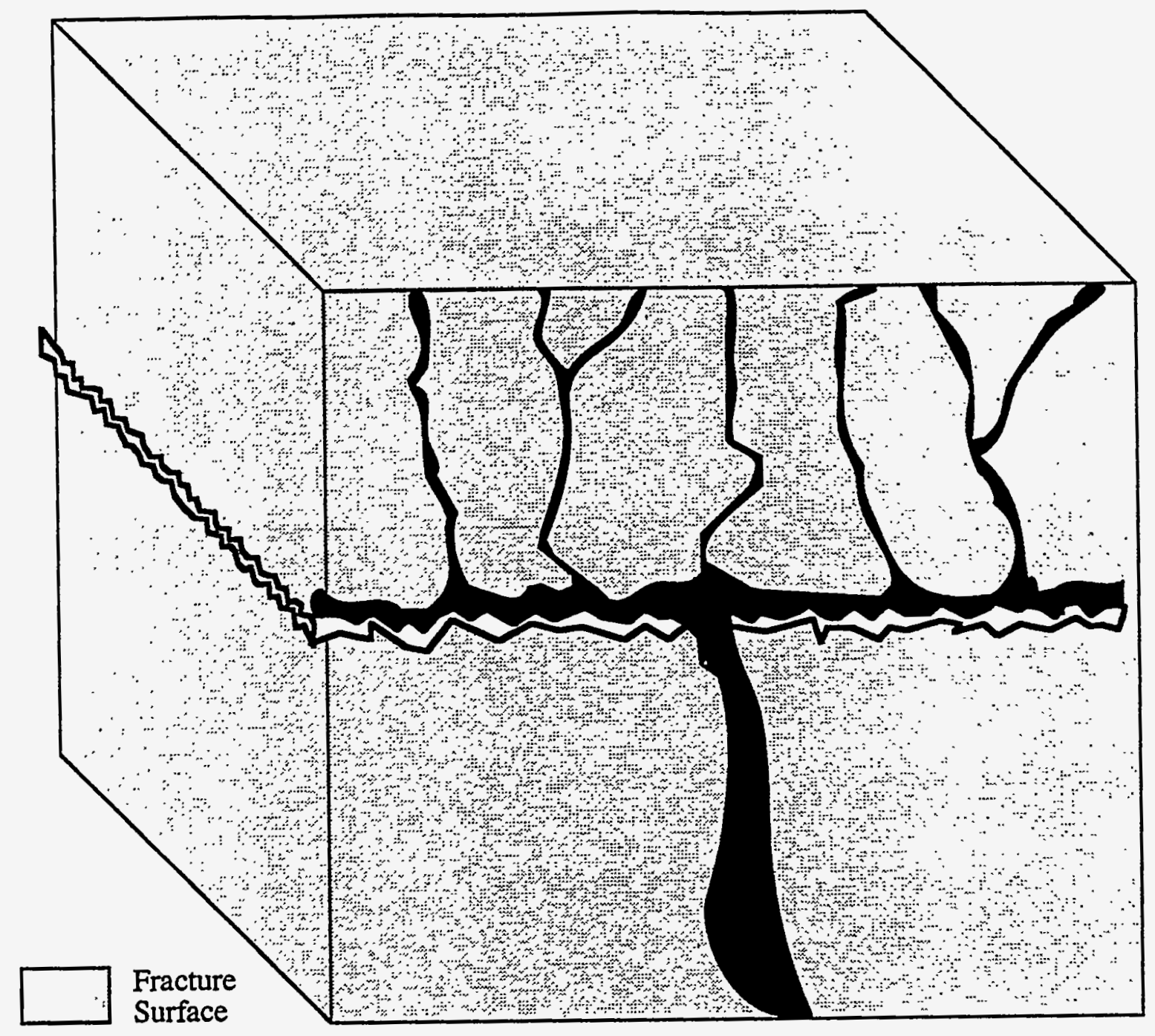

Figure 38: Confluence and merger of fingers in a fracture network: This conceptual drawing shows two matrix blocks separated by a sub-horizontal fracture and bounded by vertical fractures. Gravity-driven fingers moving down a vertical (bounding) fracture wander in response to competition between capillary and gravitational forces; when two fingers contact, they merge. Individual fingers are unable to jump the capillary boundary formed at the intersection between the vertical and sub-horizontal fracture. Water collects along the boundary forming a local tensionsatiated zone along the fracture plane. When this zone reaches a pressure sufficient to cross the capillary boundary, a drip point is formed and a single, larger finger develops. Repetition of this confluence/merger process at increasingly larger scales could act to form large scale preferential pathways through an unsaturated fractured rock mass. The spatial distribution of such pathways would be defined by a combination of flow processes and material properties; therefore, their existence and spatial location would exhibit a sensitivity to system perturbations not expected for alternative mechanisms of preferential flow (e.g., simple material heterogeneity). 


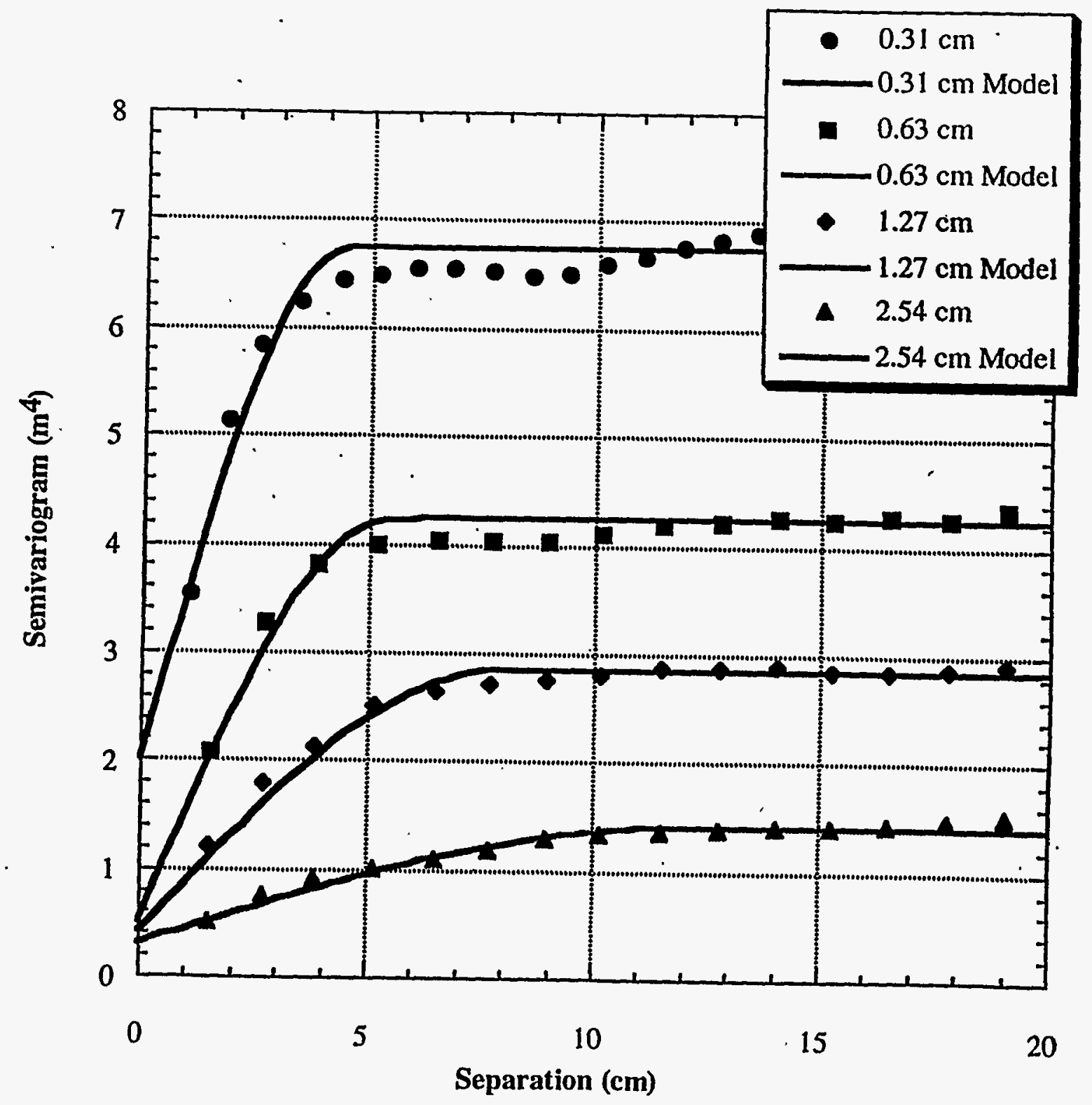

Figure 37: Quantification of spatial variability in matrix permeability: Up-scaling of gas permeability measurements also influences behavior of the multivariate statistics. Semi-variograms for each of the five tip seals measured on the Tiva Canyon sample (se Figure 35 ) are given. Note that the semi-variogram range increases in proportion to the increasing radius of the measurement (Tidwell, 1995). (2.54 cm tip). 


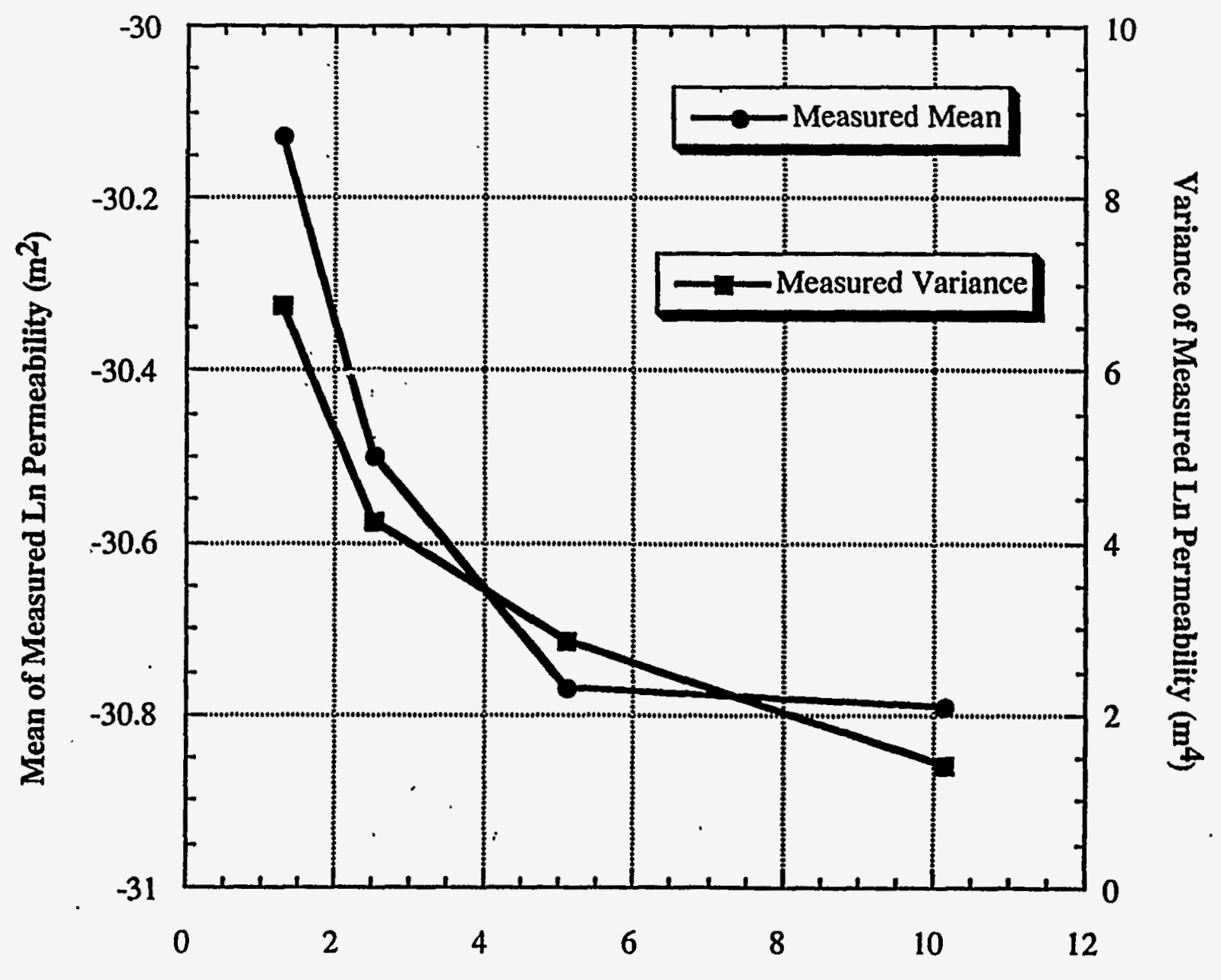

Tip Seal Size (cm)

Figure 36: Effects of sample size on matrix permeability measurements: Up-scaling of the gas permeability measured on the Tiva Canyon Tuff (see Figure 35) results in a distinct decrease in the univariate statistics. Mean and variance of In permeability are shown as a function of tip seal size. Note that the mean permeability decreases by approximately a factor of two, while the variance decreases by six orders of magnitude for a four order of magnitude increase in sample volume (Tidwell, 1995). 


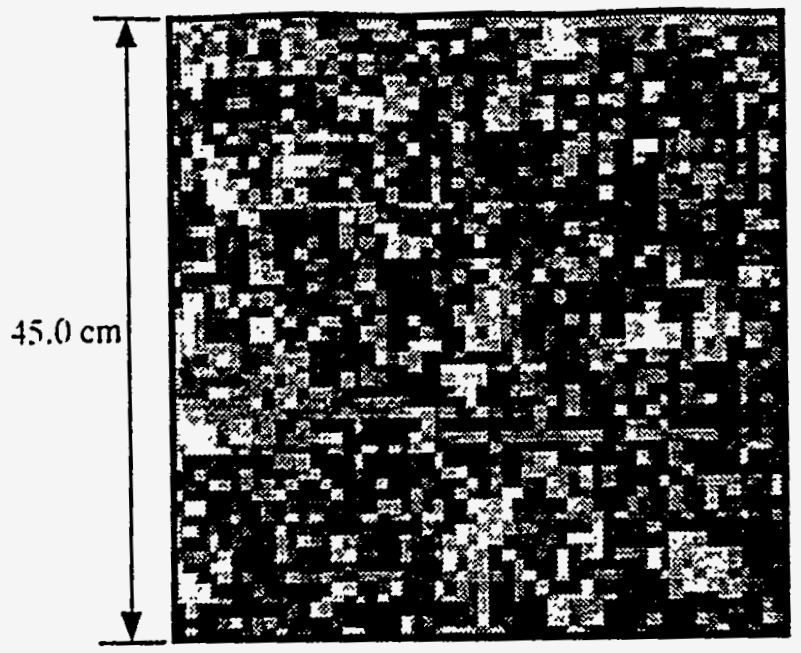

a)

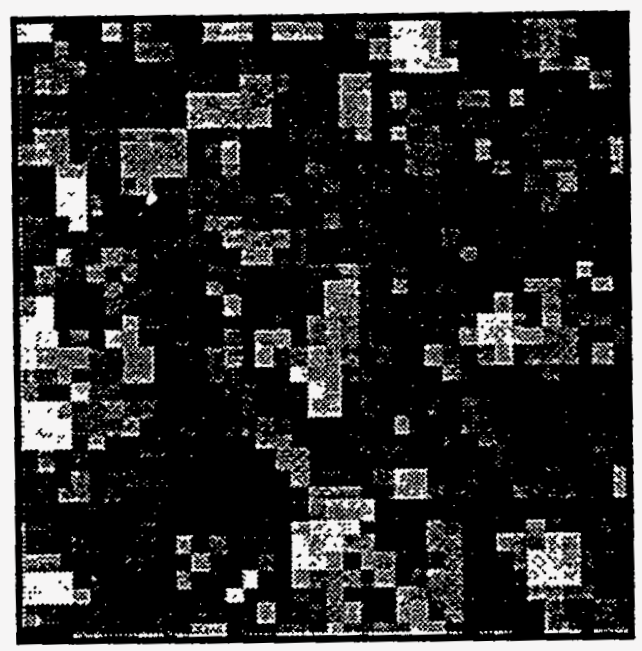

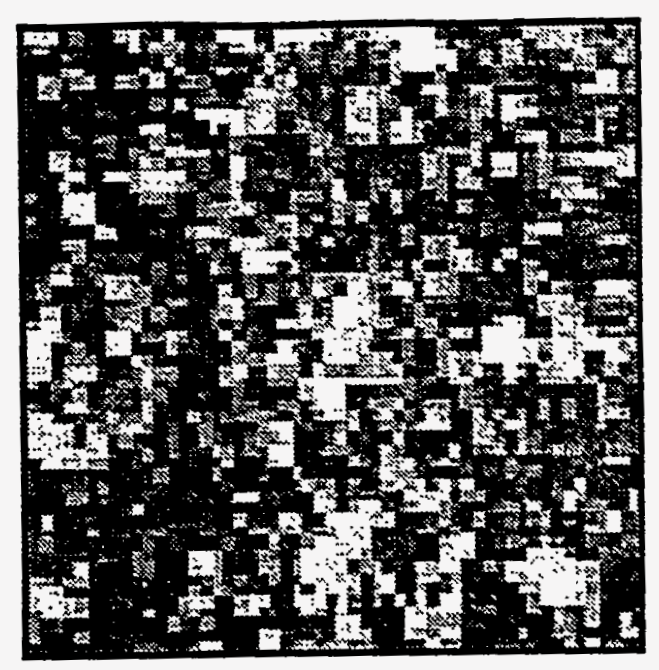

b)

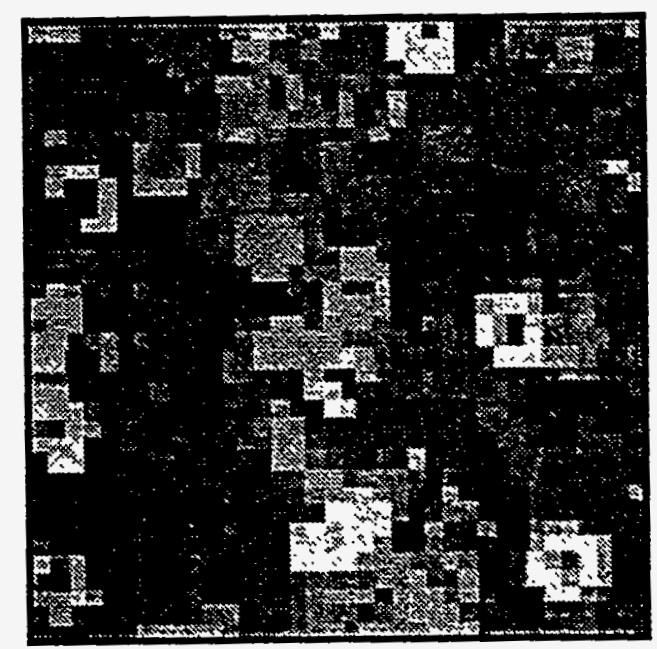

c)

d).

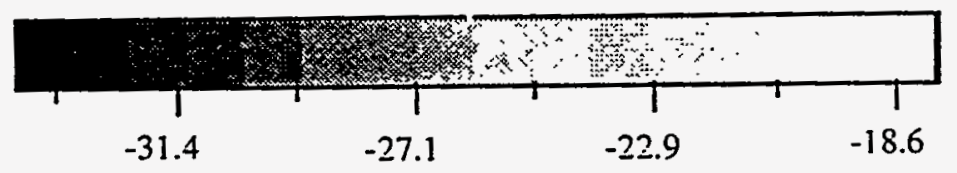

Ln Permeability $\left(\mathrm{m}^{2}\right)$

Figure 35: Matrix block heterogeneity: The scale dependency of effective media properties has important implications for a wide range of performance assessment calculations; however, relatively linle physical investigation of this effect has been accomplished. To help meet this need, gas permeability fields have been measured at different measurement scales on a block of tuff (1.2 by 1.2 by $(0.75 \mathrm{~m}$ ) collected from the Upper Cliff microstratigraphic unit of the Tiva Canyon Mcmber of the Paintbrush Tuff at Yucca Mountain. Permeability is measured by compressing a tip seal against the rock surface and injecting gas (nitrogen) at a constant pressure into the rock. Permeability is calculated based on the measured flow rate, injection pressure, and seal geometry. Different sized tip seals are used to measure gas permeability at different sample volumes. Shown are the permeability fields for tip seals with inner radii of a) 0.31, b) 0.63 , c) 1.27 , and d) $2.54 \mathrm{~cm}$. Note the distinct smoothing in the field as sample volume is increased (Tidwell, 1995). 


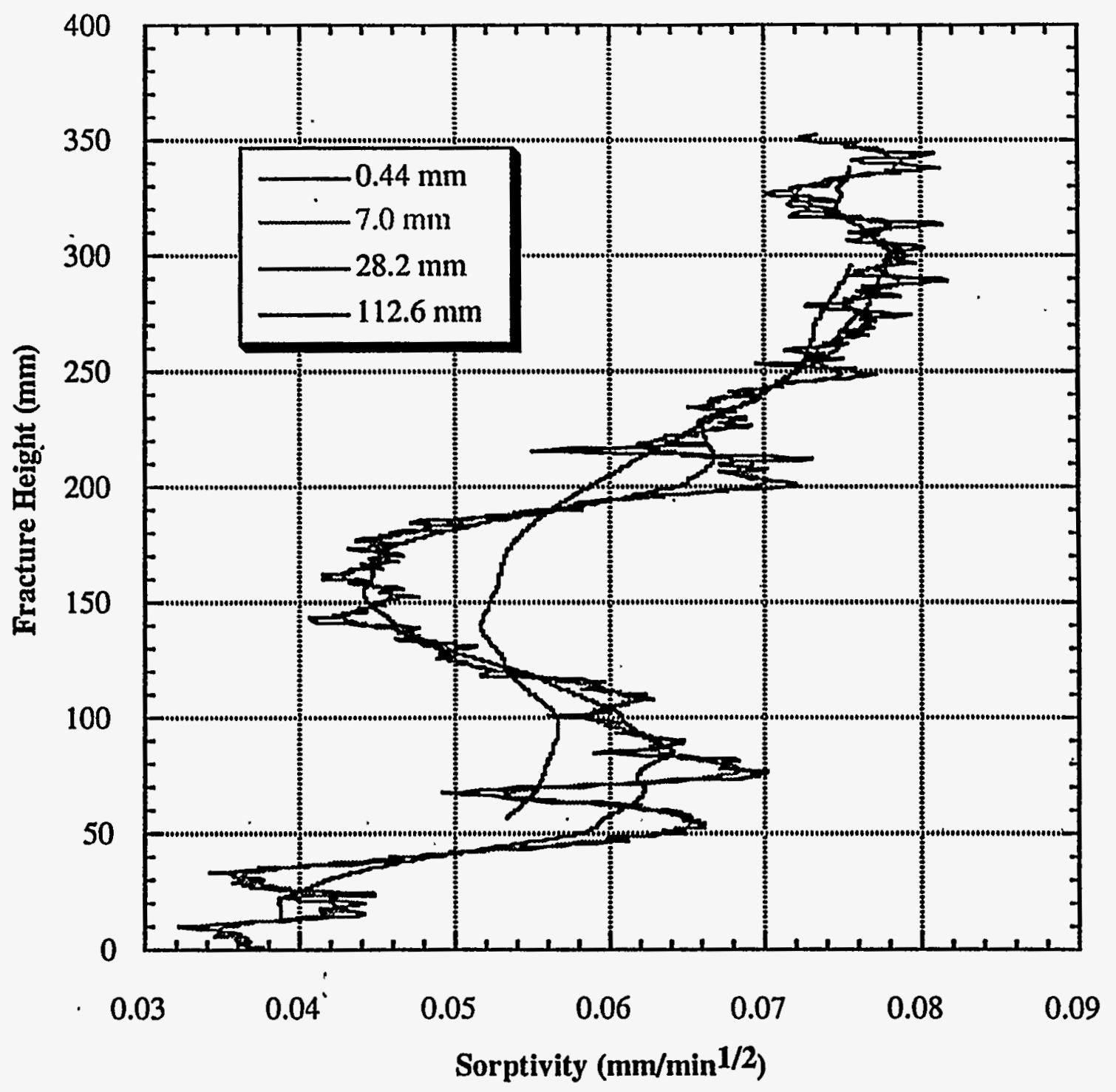

Figure 34: Matrix imbibition from a flowing vertical fracture, sorptivity measurements: Sorptivity is a measure of capillarity that linearly relates the volume of water imbibed per cross-sectional area from a constant supply pressure surface in a one-dimensional, homogeneous system to the square root of time. Measurements of imbibed water volume as a function of height for the experiment in Figure 31 show a clear square root of time dependence, thereby allowing the calculation of the sorptivity as a function of height. Even though the matrix (Figure 32a) is characterized by considerable heterogeneity (hydraulic conductivity varied by five orders of magnitude), we find that matrix imbibition can be modeled well by the sorptivity. Traces are presented for four different measurement resolutions corresponding to running fracture lengths of $0.44,7.0,28.2$, and $112.67 \mathrm{~mm}$ (Tidwell, Glass, and Peplinski, 1995). 

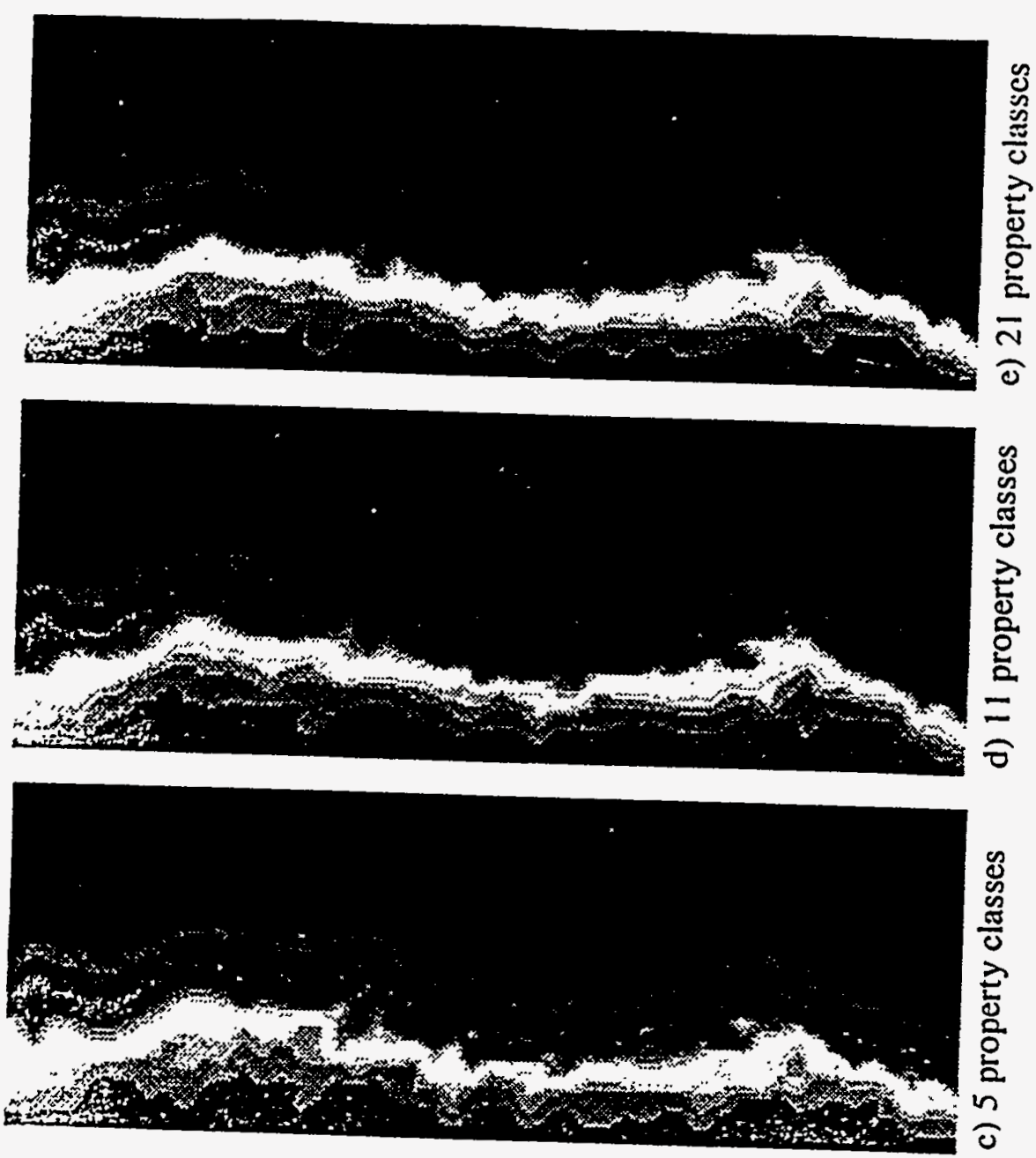

\section{$\sin 2$}

\section{istion}

3.:-

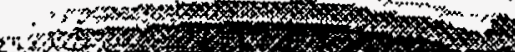

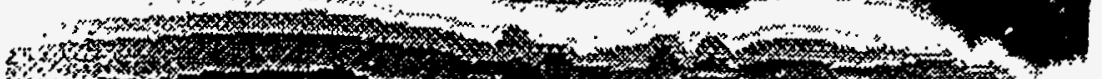
(2)
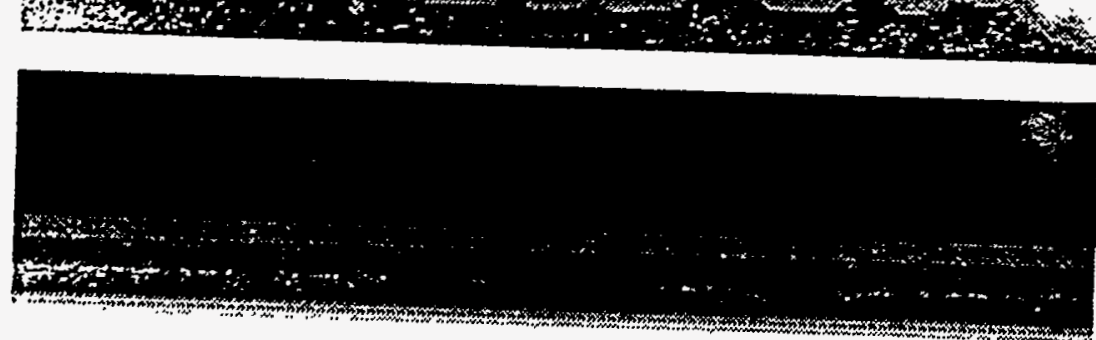

$10 \%$ on No :-10

\section{$\frac{0}{0}$}

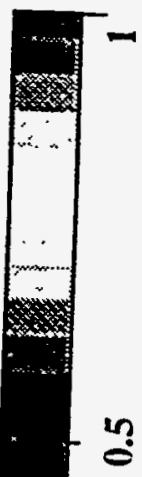

בे

$\stackrel{n}{0}$

范

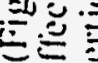

的紊竎

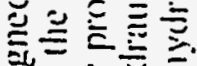

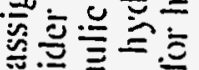

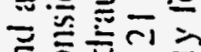

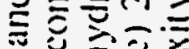

$\bar{z}=\bar{E}$

氞㤩

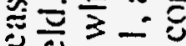

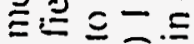

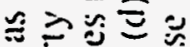

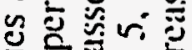

등은 인

융 चलि

흐르으응

$x \overline{5}=\frac{4}{4}$

志三ニ方

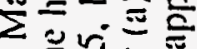

㭉的立

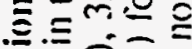

可矛示

폻응

政守

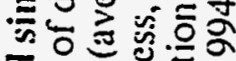

馬二昰

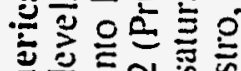

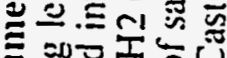

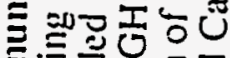

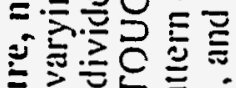
三三.

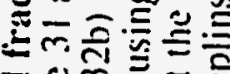

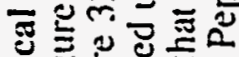

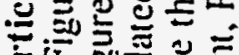

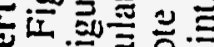

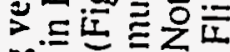

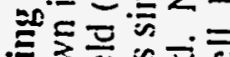

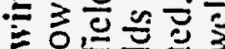

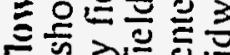
글

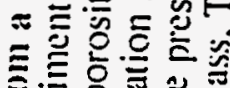

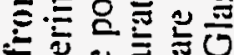

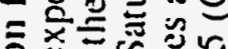

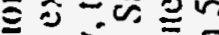
至主主 言言要要 을 플 일

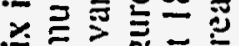

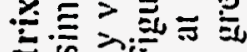

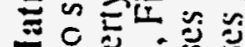

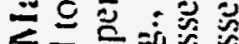

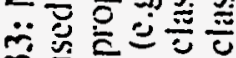

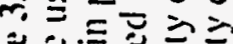

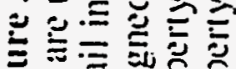

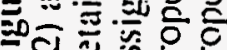

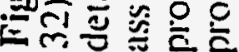




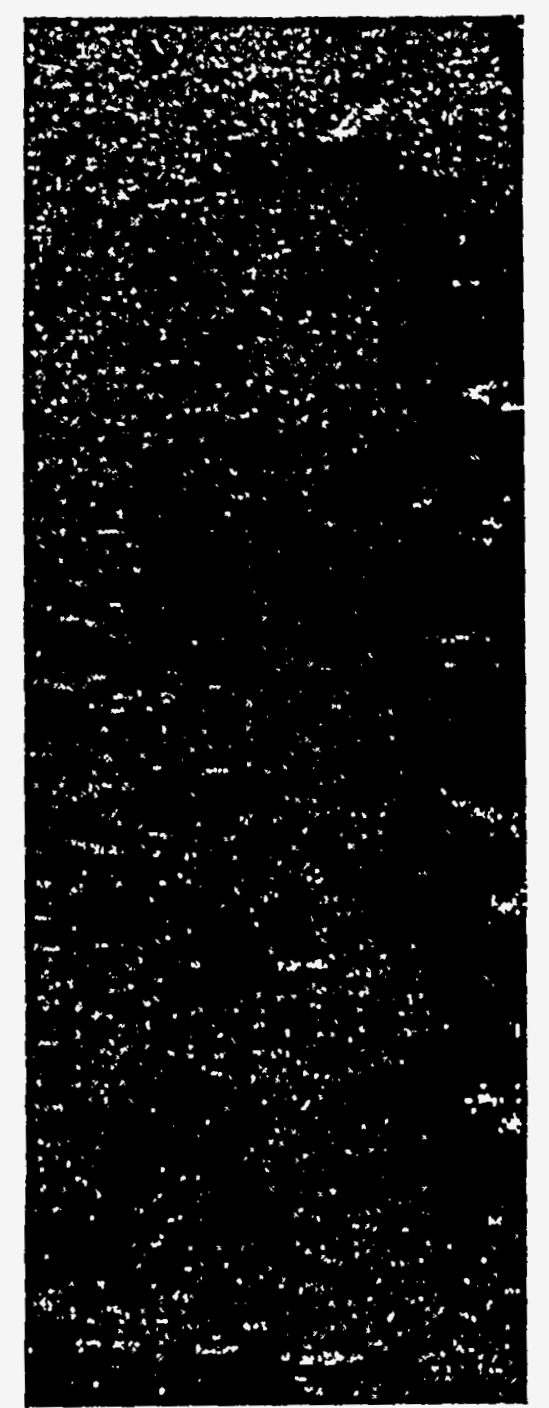

a) normalized porosity $(800 \times 288$ points)

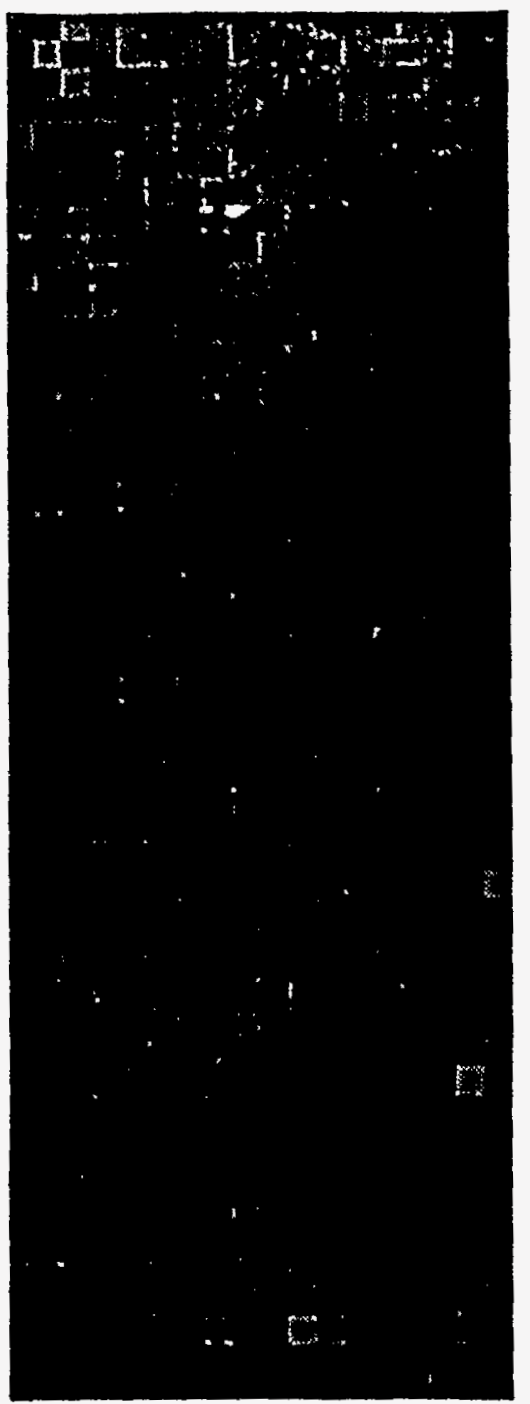

b) normalized porosity (50 x 18 points)

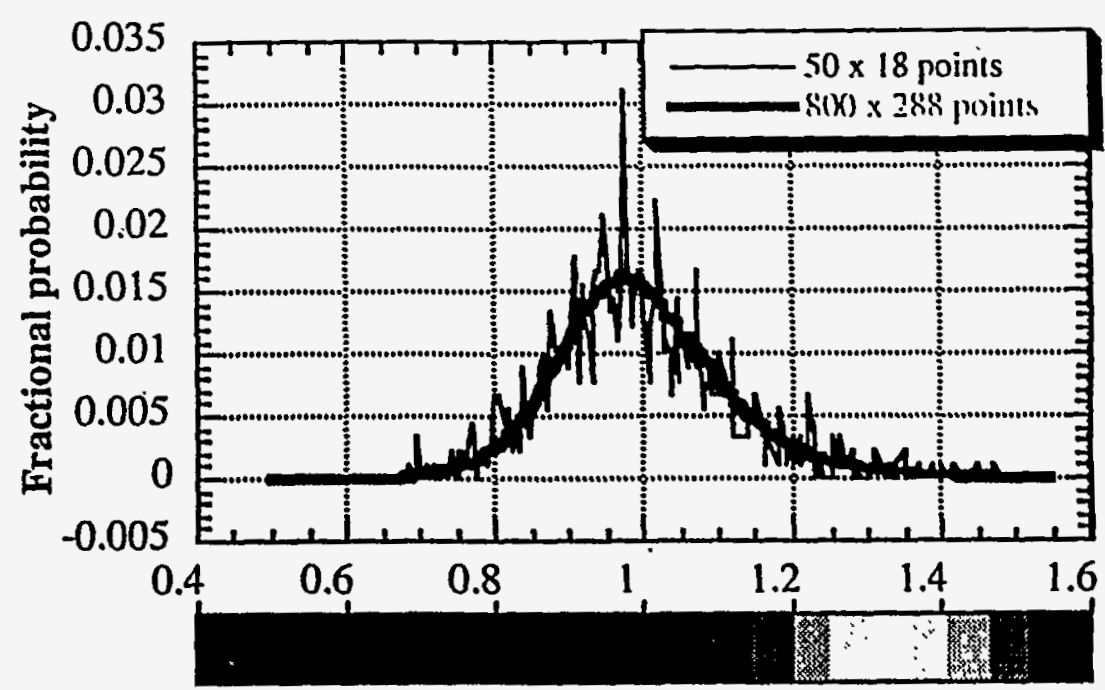

c) porosity distributions

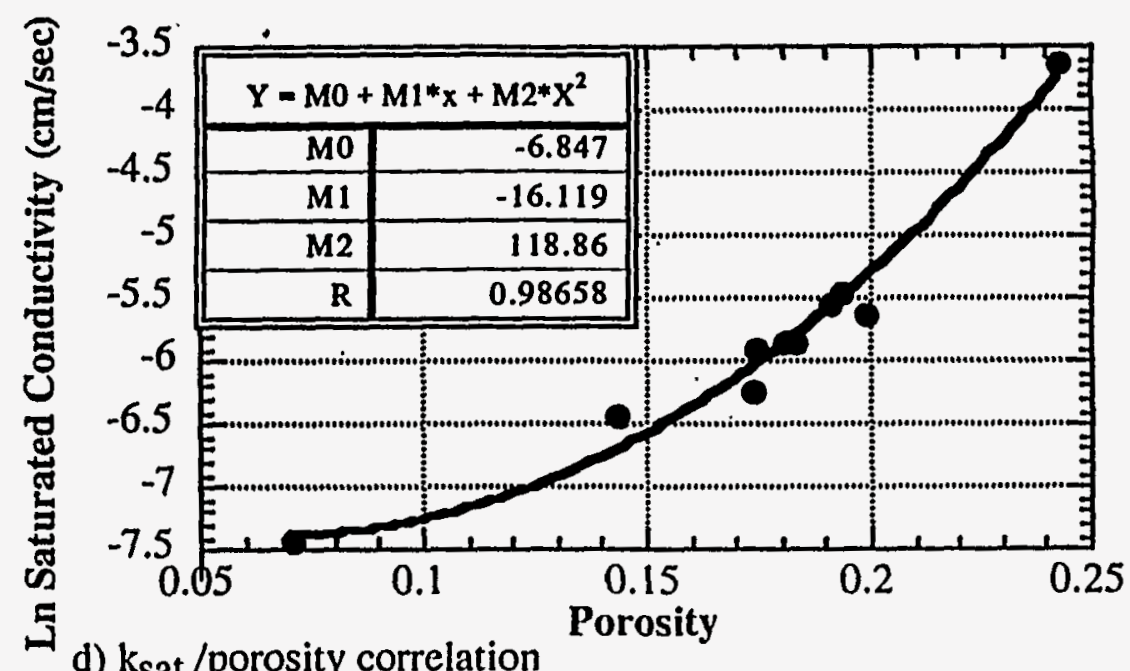

d) $\mathrm{k}_{\text {sat }} /$ porosity correlation

Figure 32: Matrix imbibition from a flowing vertical fracture, property characterization: X-ray imaging and linear absorption theory are used to acquire the normalized porosity field (porosity scaled by the mean) for the right hand slab of the tuff inatrix imbibition experiment shown in Figure 31 . The normalized porosity field at full resolution ( 800 by 288 points) shown in (a) is averaged to yield the porosity field at the desired analysis scale ( 50 by 18 points) shown as (b); the corresponding porosity distributions are shown as (c). Core scale ( $2.5 \mathrm{~cm}$ diameter) laboratory measurements of porosity and relevant hydraulic properties (saturated hydraulic conductivity, primary drainage curve of the pressure saturation relation) were performed on cuttings from the slab edges. Observed correlations between laboratory measurements of porosity and the various hydraulic properties.(e.g., see d) are then used to assign relative permeability and pressure saturation relations to each point on the slab for numerical simulation (see Figure 33). The relative permeability is modeled using the mapping of Mualem (1976) (Glass, Tidwell, Flint, Peplinski, and Castro, 1994). 


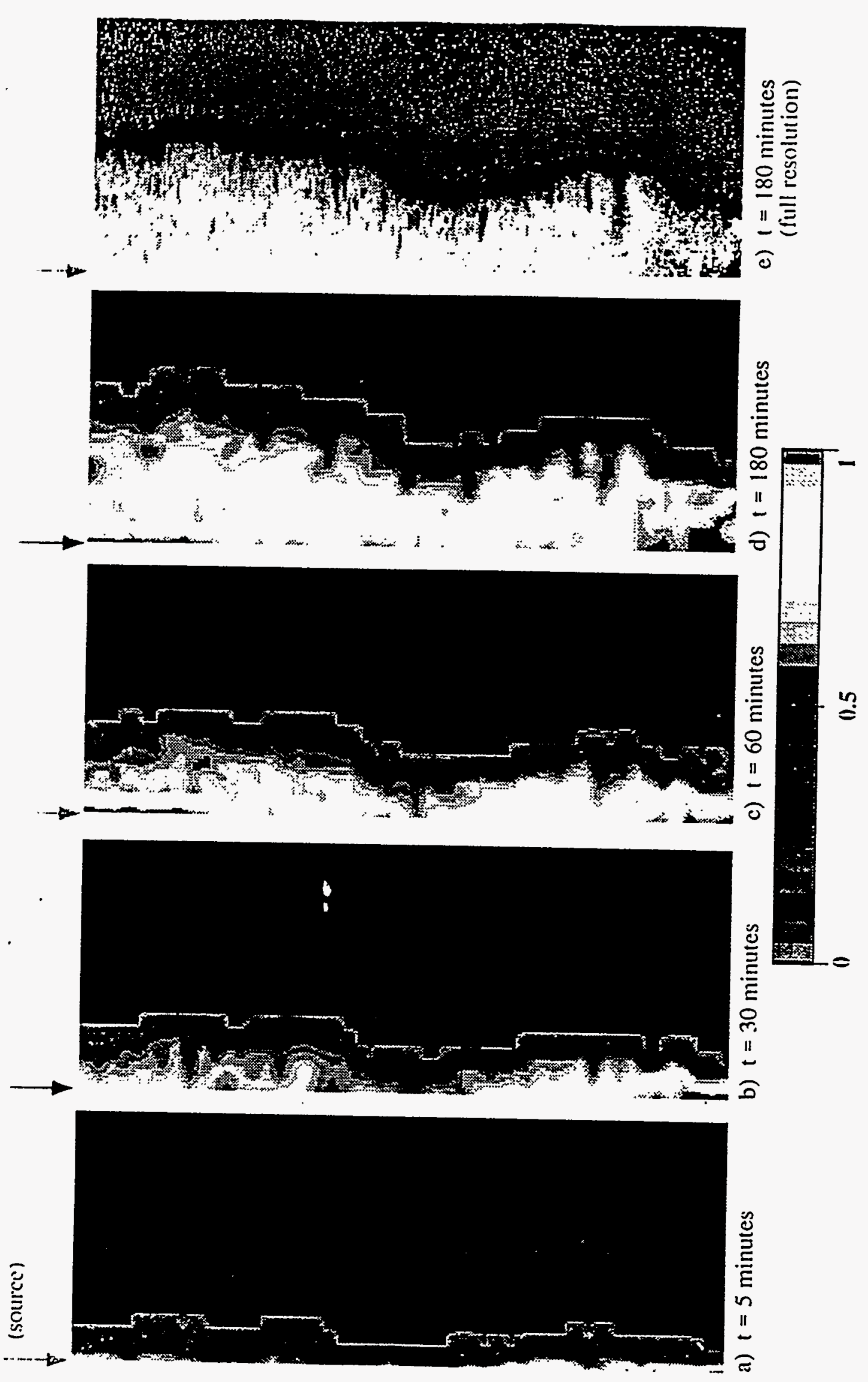

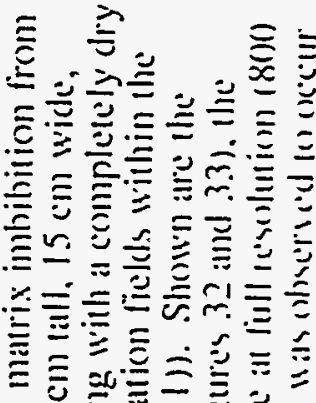

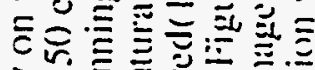

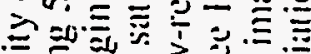

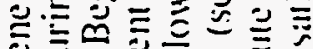

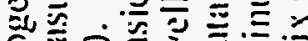

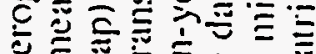

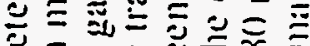
三

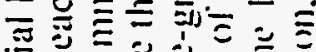
는

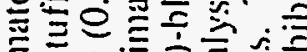

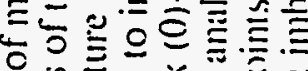

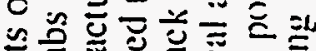

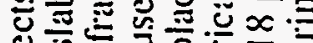

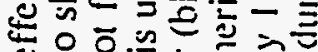

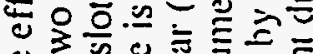

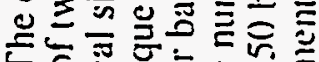

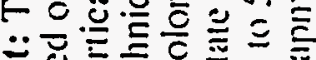

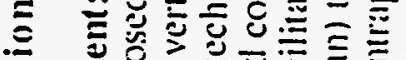

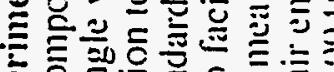

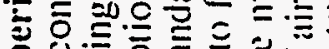

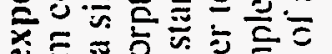

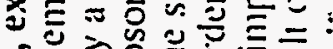

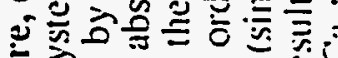

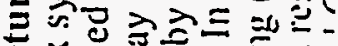
즐

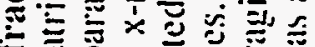

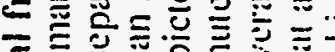

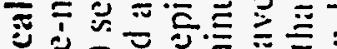

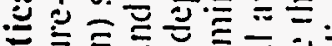

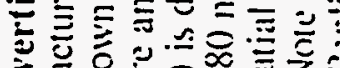

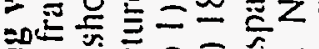

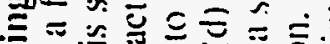

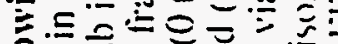

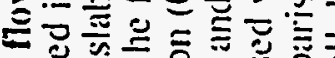
๘

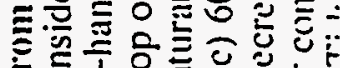

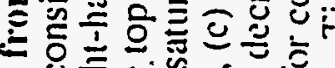

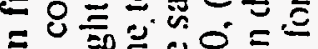

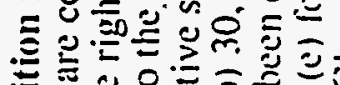

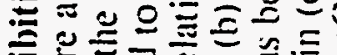

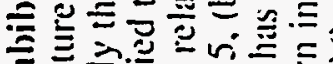

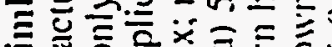

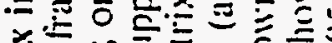

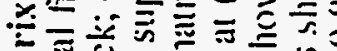

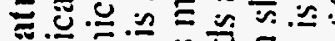

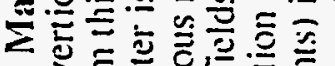

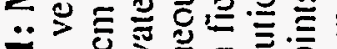
$\ddot{r_{1}}=$

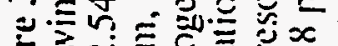

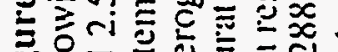

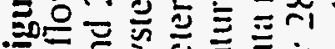

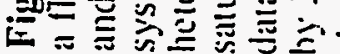




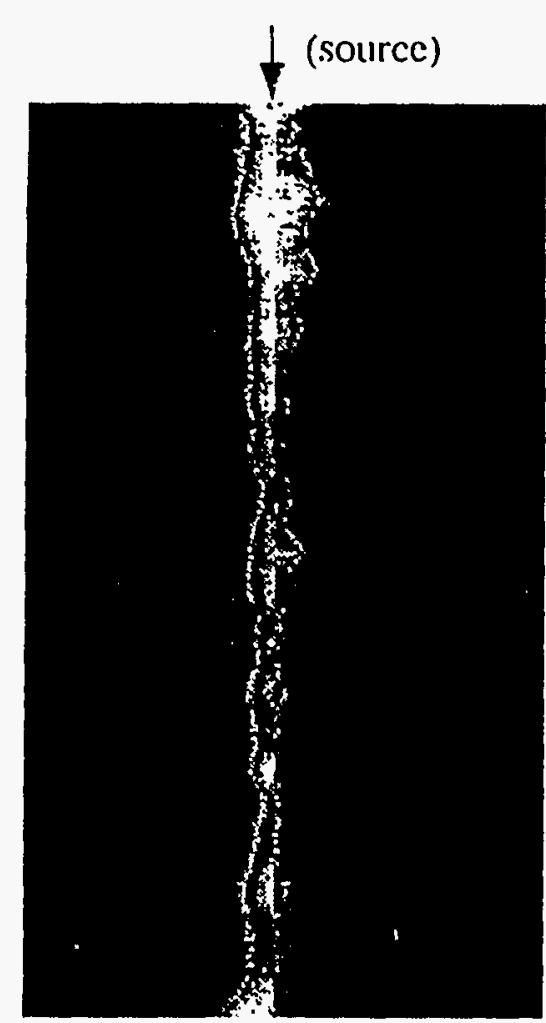

a) $t=11$ minutes

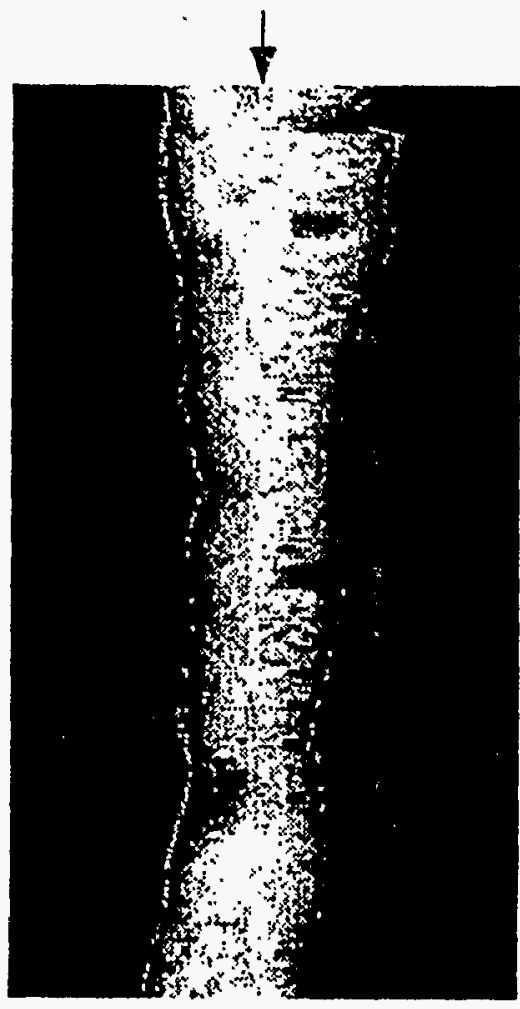

b) $\mathrm{t}=176$ minutes

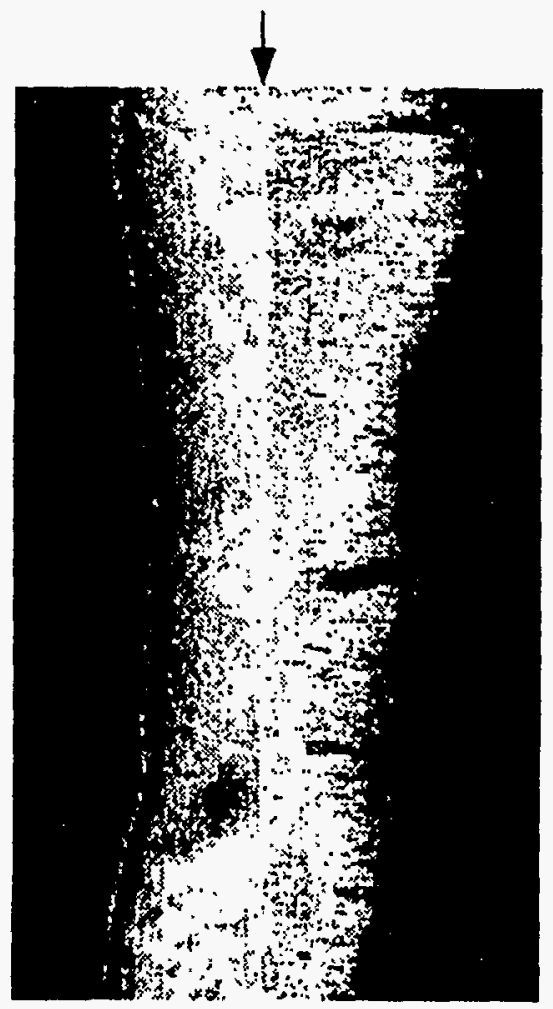

c) $\mathrm{t}=\mathbf{3 5 6}$ minutes

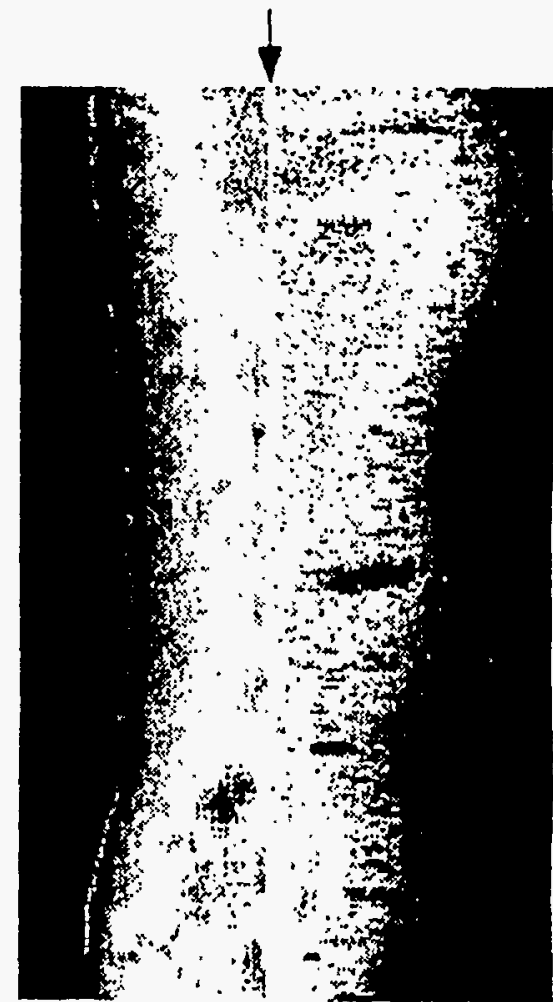

d) $t=476$ minutes

Figure 30: Imbibition of water into a fracture-matrix system, large fracture to matrix permeability ratio: The ratio of fracture to matrix permeability plays a significant role in defining the nature of fluid interactions between a vertical fracture and the adjacent porous matrix. Where the fracture permeability is large with respect to the matrix, water supplied at the top of a vertical fracture al zero pressure moves rapidly through the fracture and is accompanied by very slow and relatively uniform wetting of the matrix from along the fracture length. Horizontal nature of the imbibition process implies that gravitational effects on matrix flow are negligible. The fracture-matrix system shown here is composed of two slabs of tuff (each measuring $14 \mathrm{~cm}$ tall, $10.2 \mathrm{~cm}$ wide, and $2.5 \mathrm{~cm}$ (hick) separated by a single vertical slot fracture $(0.1 \mathrm{~mm}$ gap). To avoid anomalous pressure build-up, all boundarics are left open to atmospheric pressure.

Beginning with a completely dry system, water is supplied to the top of the fracture and $x$-ray absorption is used to image the transient saturation fields during imbibition; relative saturation (0 to 1 ) is depicted by the standard color bar (black (0)-blue-green-yellow-redt 1 )). Images are shown for (a) 11 , (b) 176, (c) 356 , and (d) 476 minutes after initiation of flow into the fracture (Foltz, Ticlwell, (Blass, and Sobolick, 1993). 


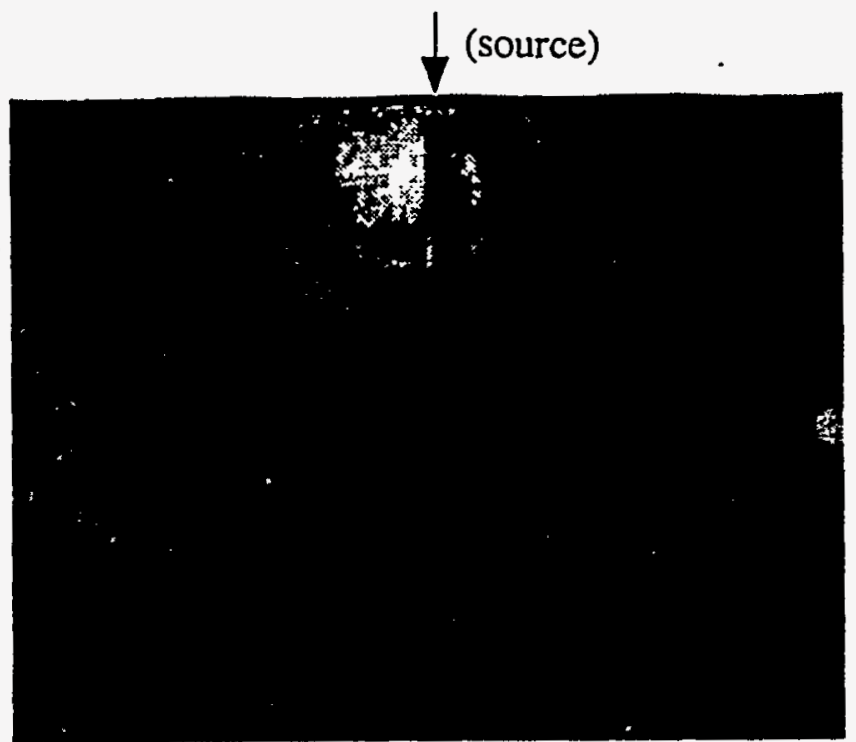

a) $t=4$ minutes

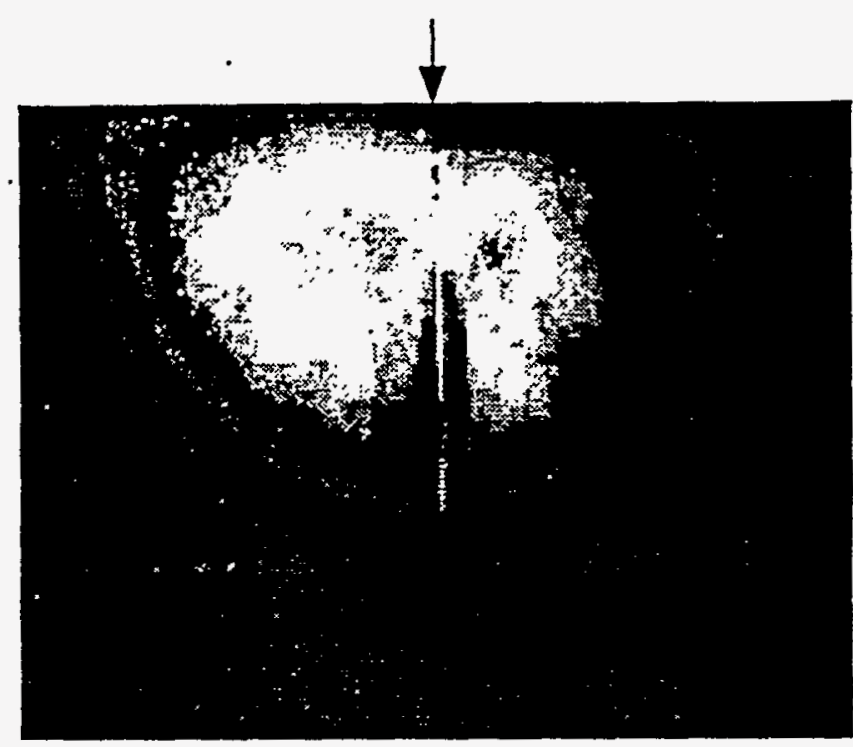

c) $\mathrm{l}=\cdot 10$ minutes

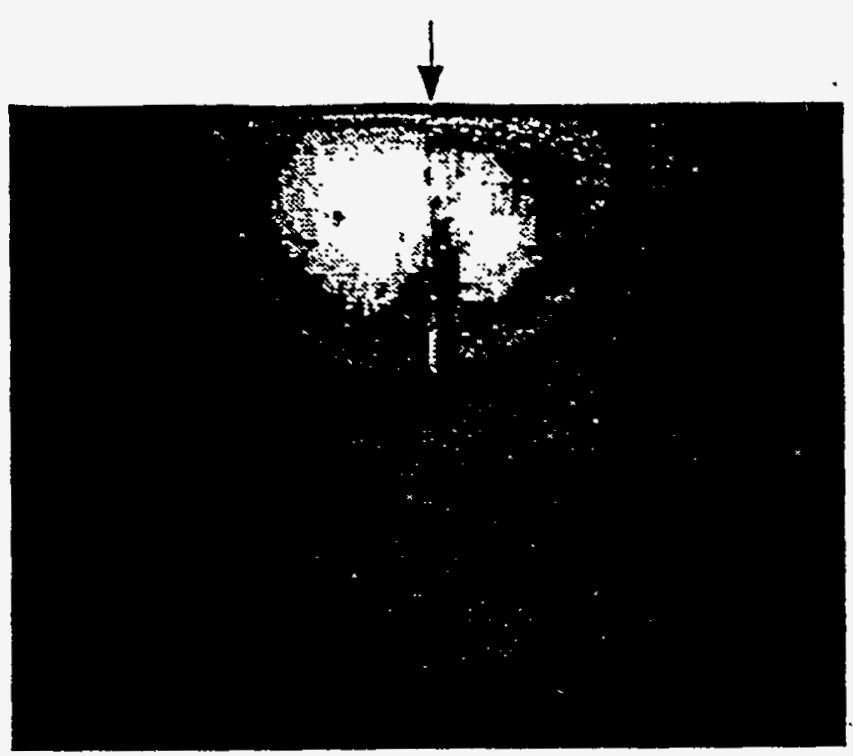

b) $t=6$ minutes

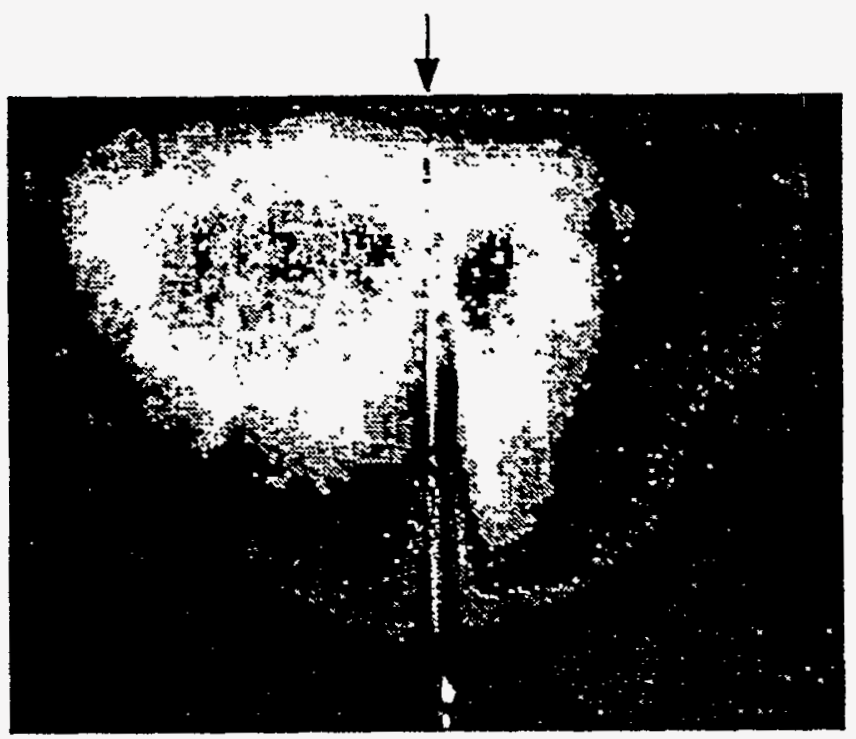

d) $t=15$ minutes

Figure 29: Imbibition of water into a fracture-matrix system, small fracture to matrix permeability ratio: The ratio of fracture to matrix permeability plays a significant role in defining the nature of fluid interactions between a vertical fracture and the adjacent porous matrix. In the fracture-matrix system shown, matrix permeability is of the same order as the fracture permeability. As a result, the depth of penetration of water in the fracture from a zero pressure water source located at the top of the fracture is closely matched by the depth of penetration in the matrix. The fracture-matrix system is composed of a sintered glass plate $(15$ by 10 by $0.6 \mathrm{~cm}$ ) cut by a single, vertical slot fracture $(0.1 \mathrm{~mm}$ gap, see artows). To avoid anomalous pressure build-up, all houndaries are left open to atmospheric pressure. Beginning with a completely dry system, water is supplied to the top of the fracture and transmitted light imaging is used to obtain transient saturation fields during imbibition; relative saturation (0 to 1$)$ is depicted by the standard color bar (black (0)blue-yreen-yellow-red(1)). Saturation fields are shown for (a) 4, (b) 6 , (c) 10, and (d) 15 minutes following initiation of flow (Glass and Tidwell, 1991). 


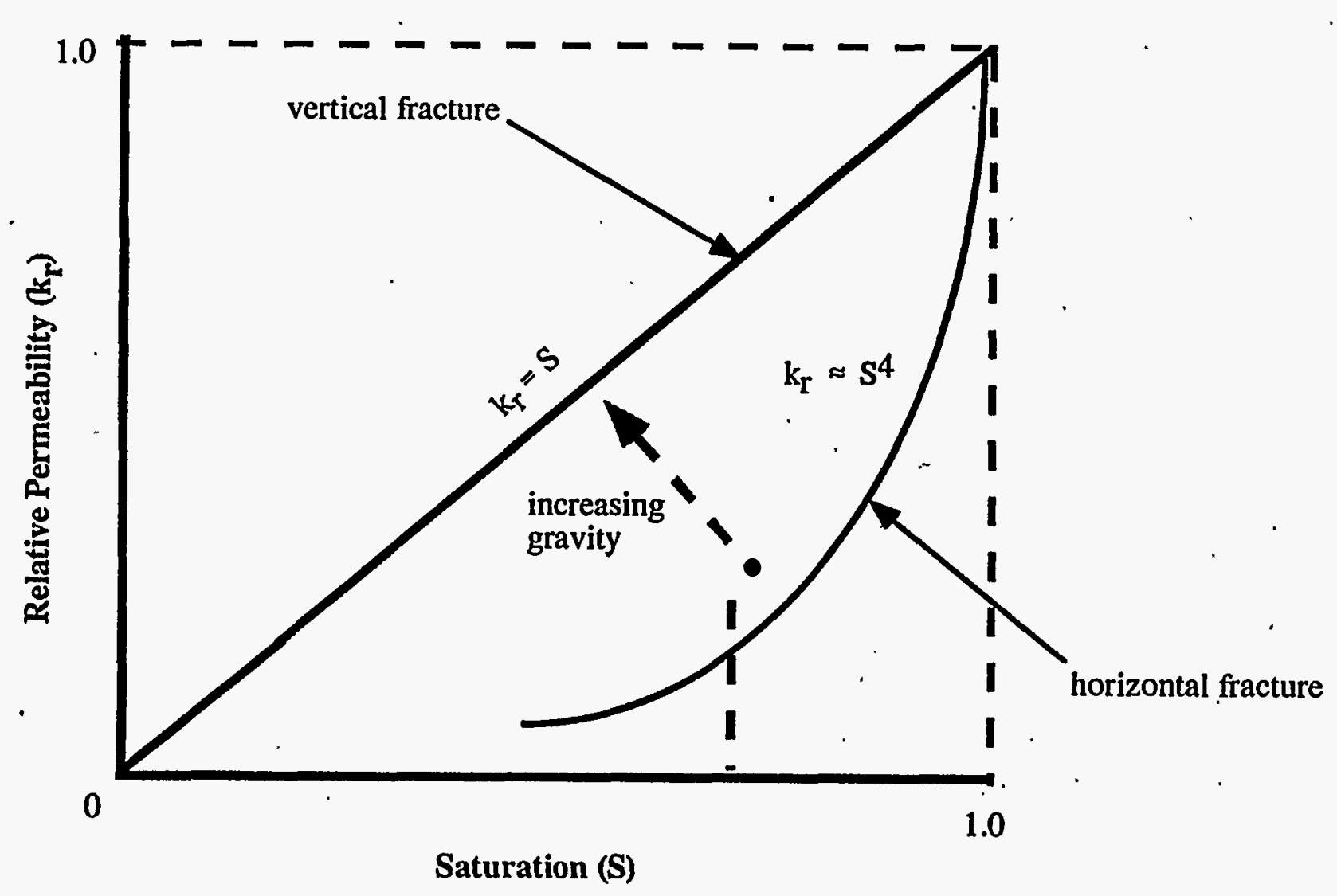

Figure 28: Effects of gravity on fracture relative permeability: Where gravity-driven fingering occurs, relative permeability $\left(k_{r}\right)$ is expected to approach saturation (S). In horizontal fractures at very low saturations, the fluid phase along the fracture will be disconnected, leading to a steady-state $\mathrm{k}_{\mathrm{r}}$ of zero. At the percolation threshold, $\mathrm{k}_{\mathrm{r}}$ will jump to a finite value (left side of green dashed line); subsequent dissolution of the air phase will cause $\mathrm{k}_{\mathrm{r}}$ to increase along a power-law function with $\mathrm{S}$. From horizontal to vertical, the relative permeability function is expected to translate as shown in the blue dashed arrow, however, this has yet to be tested experimentally. 
Dimensionless finger width:

$$
\frac{\text { measured finger width }}{\text { system width }}=\frac{W_{f}}{W_{s}}
$$

\section{Dimensionless flow:}

$$
\frac{\text { supply flow }}{\text { full width saturated flow }}=\frac{Q}{W_{s} \hat{a} K_{s} \cos \beta}
$$

where: .

$$
\begin{aligned}
W_{f} & =\text { measured finger width } \\
W_{s} & =\text { system width } \\
\hat{a} & =\text { mean fracture aperture } \\
K_{s} & =\text { saturated hydraulic conductivity of fracture } \\
\beta & =\text { inclination of fracture from vertical } \\
Q & =\text { flow rate to the finger }
\end{aligned}
$$

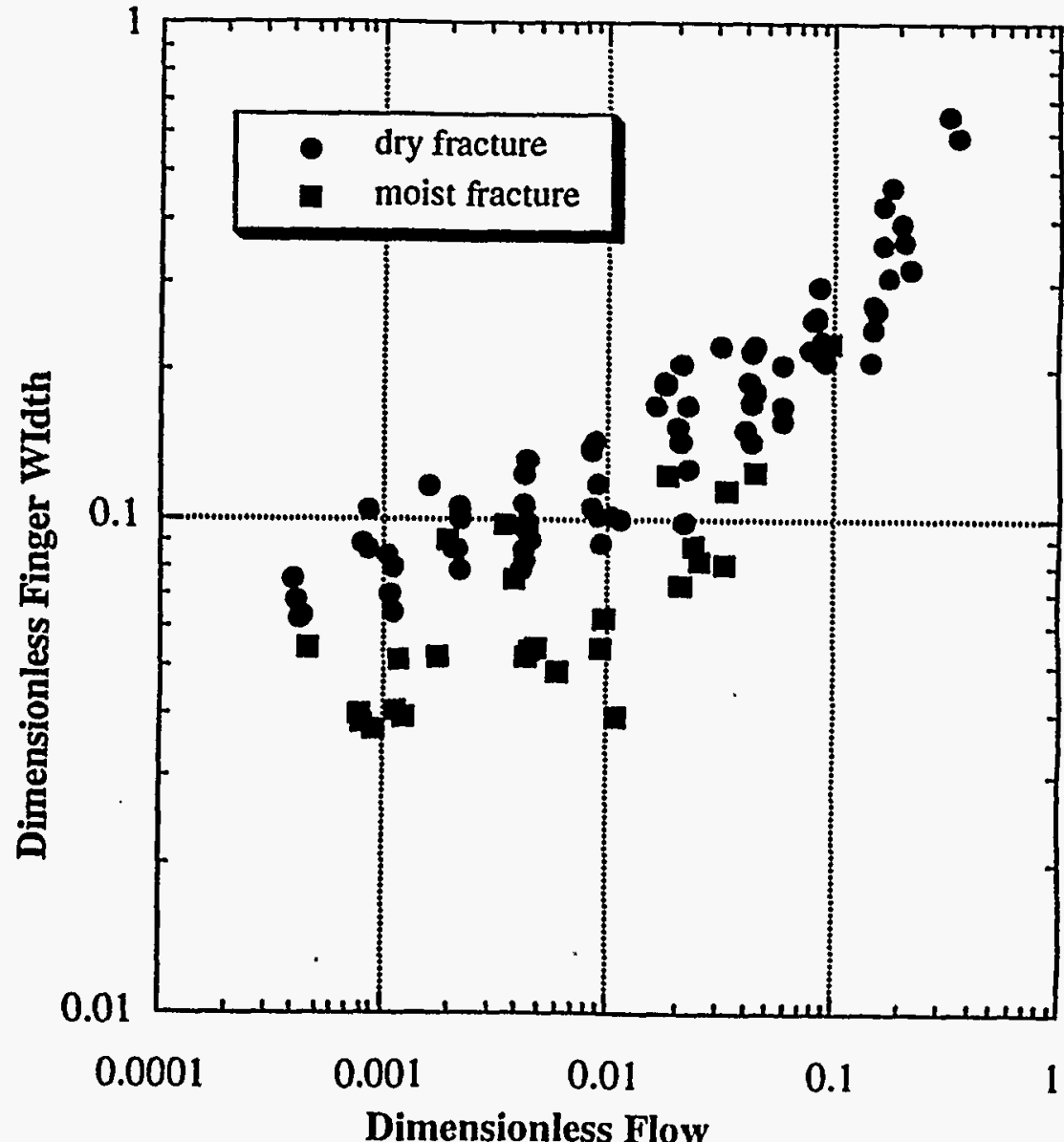

Figure 27: Comparison of finger width between dry and uniformly moist initial conditions: Statistically homogeneous (sce Figure 3 ) analog fractures $(30 \times 60 \mathrm{~cm}$ for moist, $15 \times 30 \mathrm{~cm}$ for dry) are used to explore the effects of initial moisture content (field capacity) on finger width. Individual fingers are initiated from a point source in order to provide experimental control of flow rate through the finger. Finger width under pre-wetted conditions (red squares) is observed to be generally narrower than observed under dry initial conditions (black circles). Gravity drainage of the analog fracture creates a wetted structure consisting of disconnected water blobs that are distributed about the fracture; at any given inclination, such blobs will tend toward a uniform size that is defined by competition between gravity and capillary forces. When contacted by a finger, such blobs act as instantaneous perturbations to the finger tip; blobs larger than the finger width are likely to induce finger splitting, while smaller blobs will act to reduce finger width (Nicholl, Glass, and Nguyen, 1993b). 
Dimensionless Finger Velocity:

$$
\frac{\text { measured finger velocity }}{\text { saturated flux }}=\frac{V_{f}}{K_{s} \cos \beta}
$$

Dimensionless Finger flux:

$$
\frac{\text { average finger flux }}{\text { saturated flux }}=\frac{Q}{W_{f} \hat{a}} \cdot \frac{\cdot 1}{K_{s} \cos \beta}
$$

where:

$\beta=$ fracture inclination from vertical

$\cos \beta=$ gravitational gradient

$Q=$ mass flow rate

$K_{s}=$ saturated hydraulic conductivity

$V_{f}=$ finger velocity

$W_{f}=$ finger width

$\hat{\mathbf{a}}=$ mean aperture

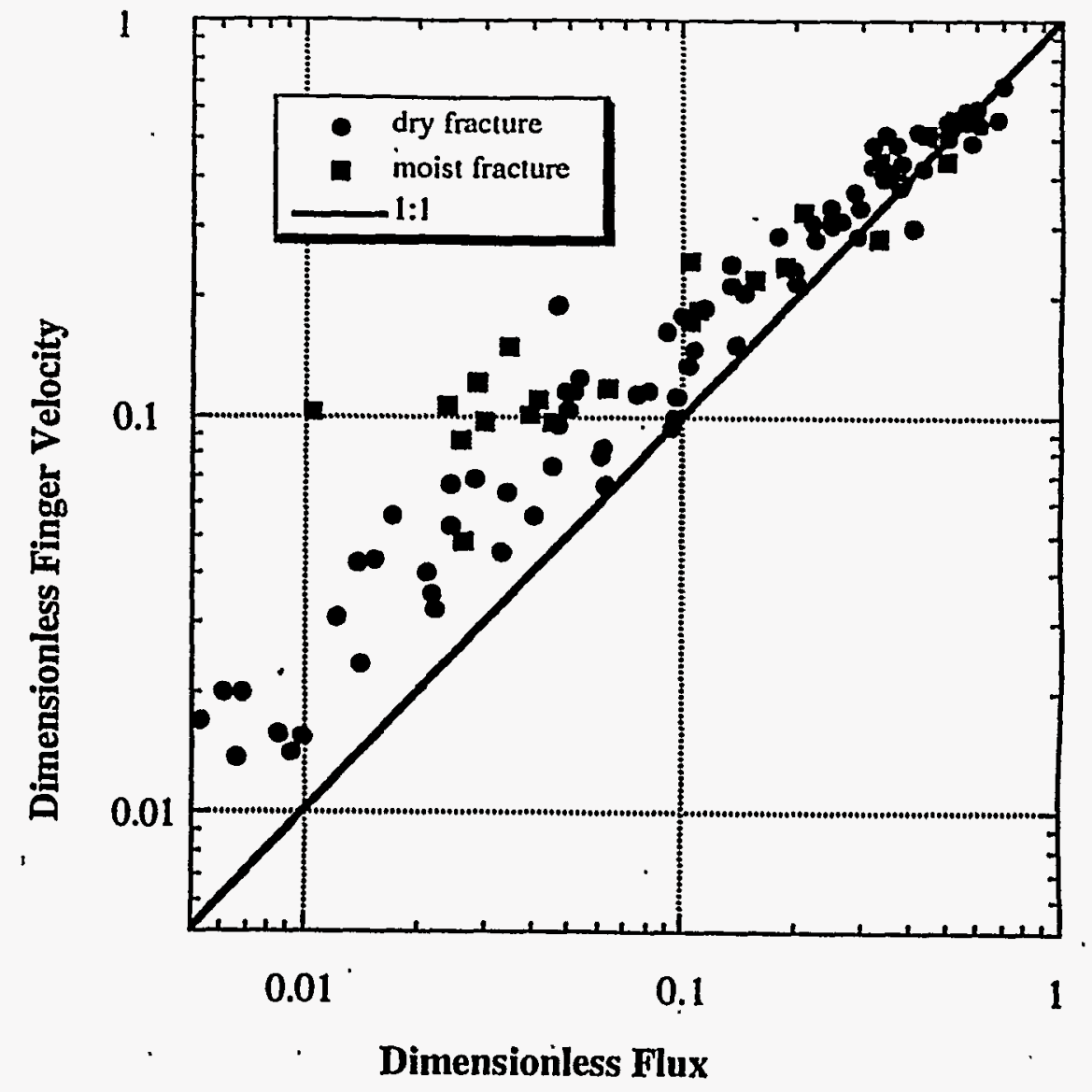

Dimensioniless Flux

Figure 26: Comparison of dimensionless finger velocity between dry and uniformly moist initial conditions: Statistically homogeneous (see Figure 3) analog fractures $(30 \times 60 \mathrm{~cm}$ for moist, $15 \times 30 \mathrm{~cm}$ for dry) are used to explore the effects of initial moisture content (field capacity) on finger velocity. Individual fingers are initiated from a point source in order to provide experimental control of flow rate through the finger. Finger velocity under pre-wetted conditions (red squares) is observed to be generally larger than that observed under dry initial conditions (black circles), particularly at small dimensionless flux. At field capacity, moisture within the analog exists primarily as isolated "blobs"; when contacted by a finger, the blob becomes the leading edge of the finger-tip. The relative significance of initial moisture content on finger velocity is observed to increase with decreasing dimensionless flux. The primary control on finger velocity at a given inclination is finger length, which is expected to decrease with dimensionless flux; as finger length becomes smaller, the impact of finite size perturbations associated with the in situ moisture field will become increasingly significant (Nicholl, Glass, and Nguyen, 1993b). 

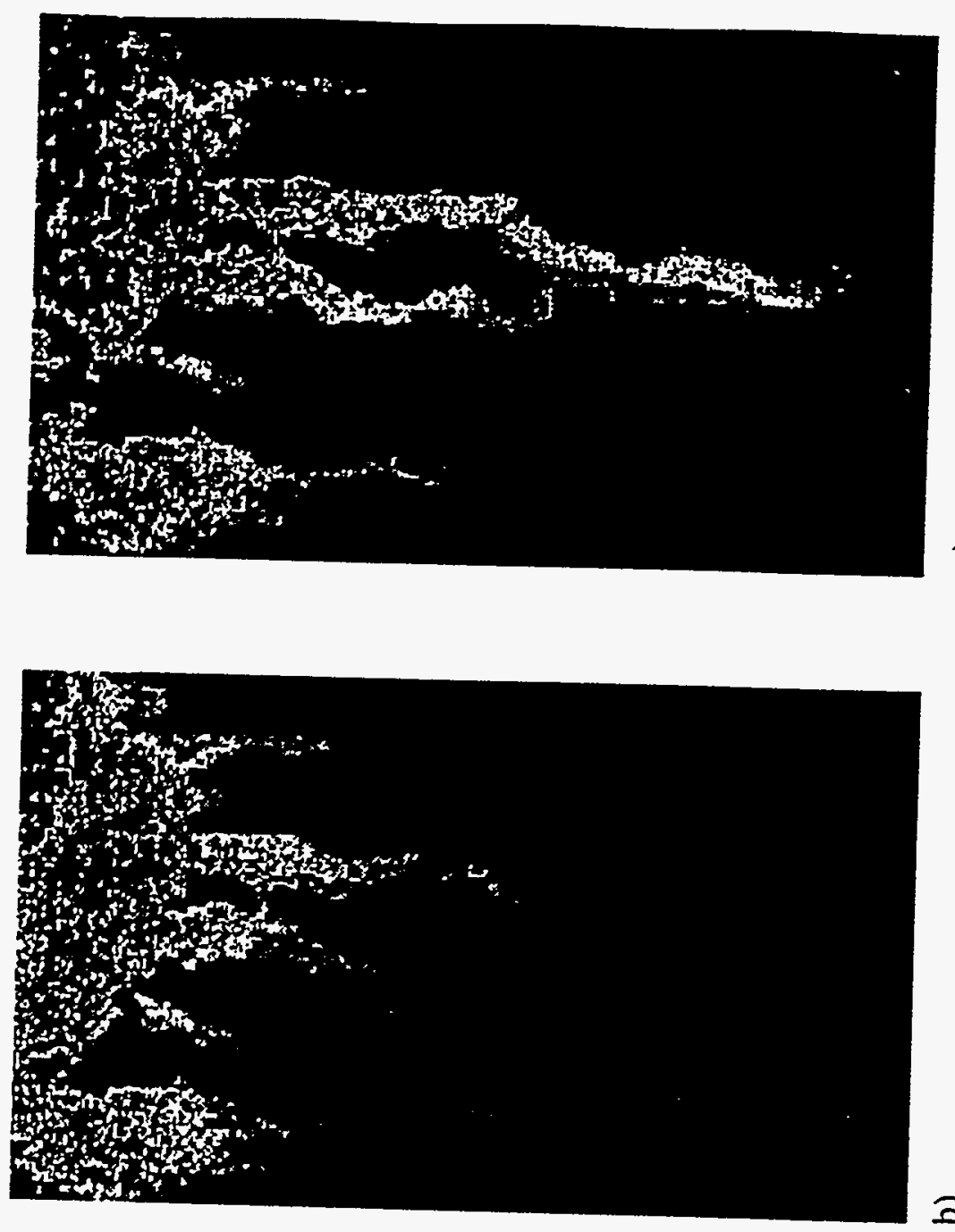

\%

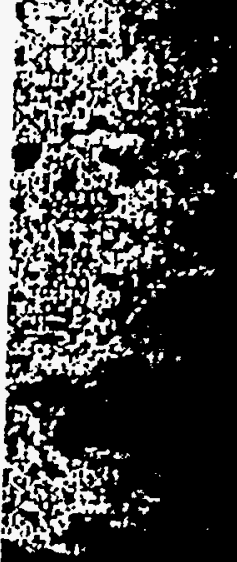

อ 


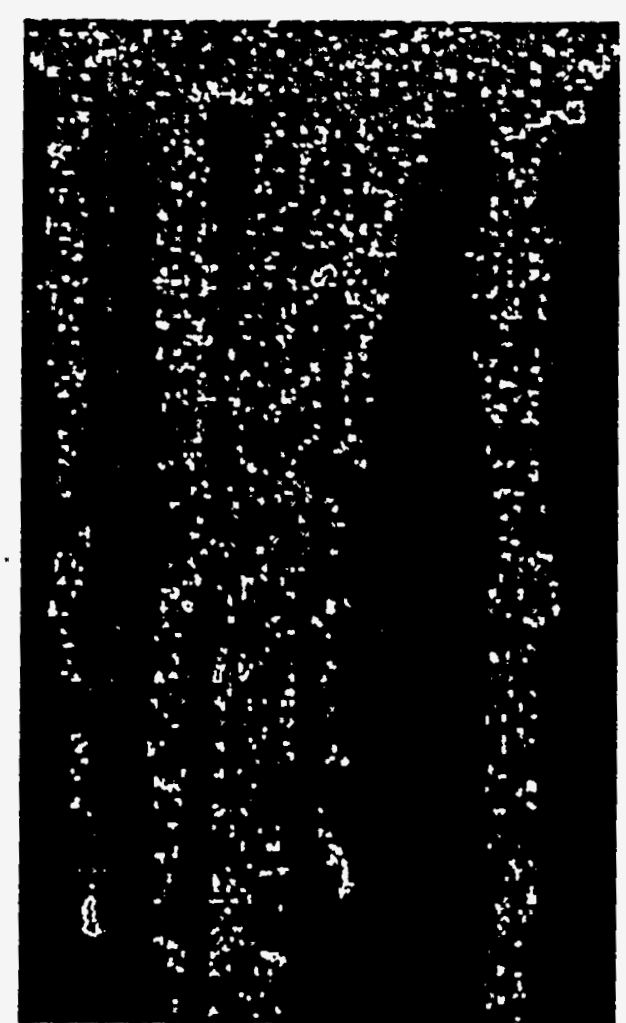

a)

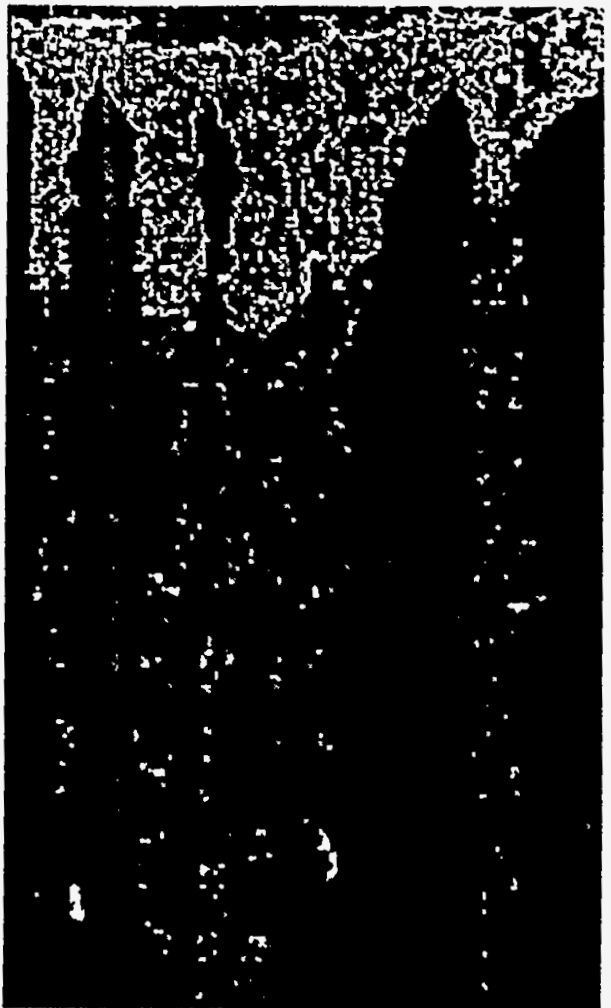

b)

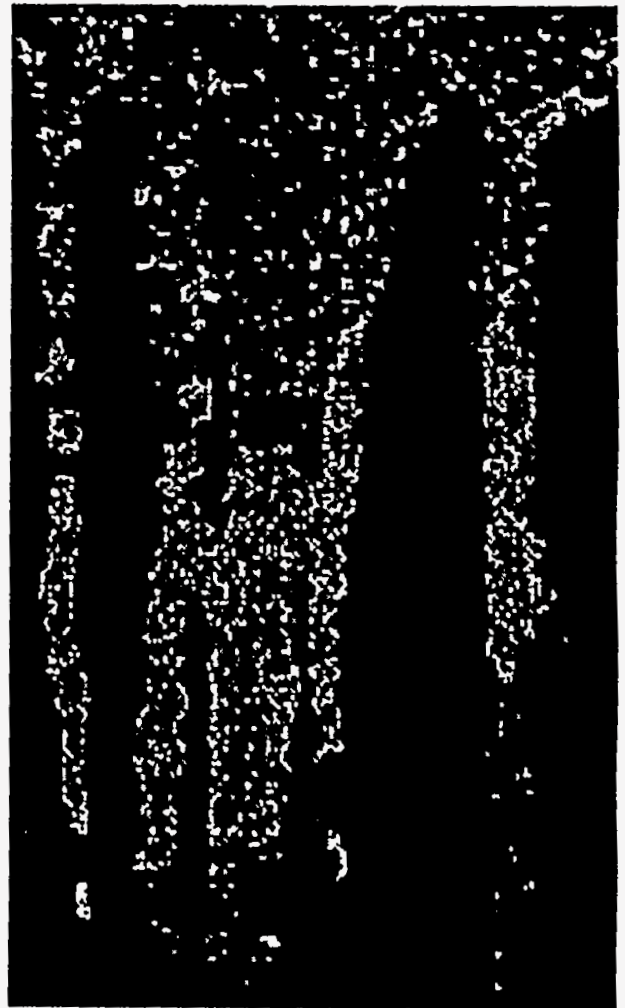

c)

Figure 24: Persistence of gravity fingers from one ponded event to the next: A statistically homogeneaus (sec Figure 3) analog fracture $(30 \times 60 \mathrm{~cm})$ inclined 60 degrecs from vertical $(\cos \beta=0.5)$ is used to consider the spatial persistence of fingers beiween subsequent ponded infiltration events. The initial condition is created by unstable redistribution following ponded infiltration under dry initial conditions (a); black areas are dry, purple indicates the moist finger pathways, and red/blue indicates water fully spanning the fracture aperture. At the end of the first ponded event, each finger has either left the system, or terminated in a small saturated zone that is pinned in place by capillary forces. Two such saturated zones are seen as red regions near the bottom of (a); in a larger system, the other fingers would form similar termination's at greater depth. Subsequent ponded infiltration is observed to preferentially follow the cxisting wetted structure (b), and to finger within that wetted structure (c). The advancing fingers $(b, c)$ lose very little, or no watce prior to reaching the end of the pre-wetted paths, where they join with the pinned saturated zones and continuc downward into the diry fracture. Fingers were observed to be less compact, contain more entrapped air, and be more complex than those observed under dry initial conditions (Nicholl, Glass, and Nguyen, 1993b). 


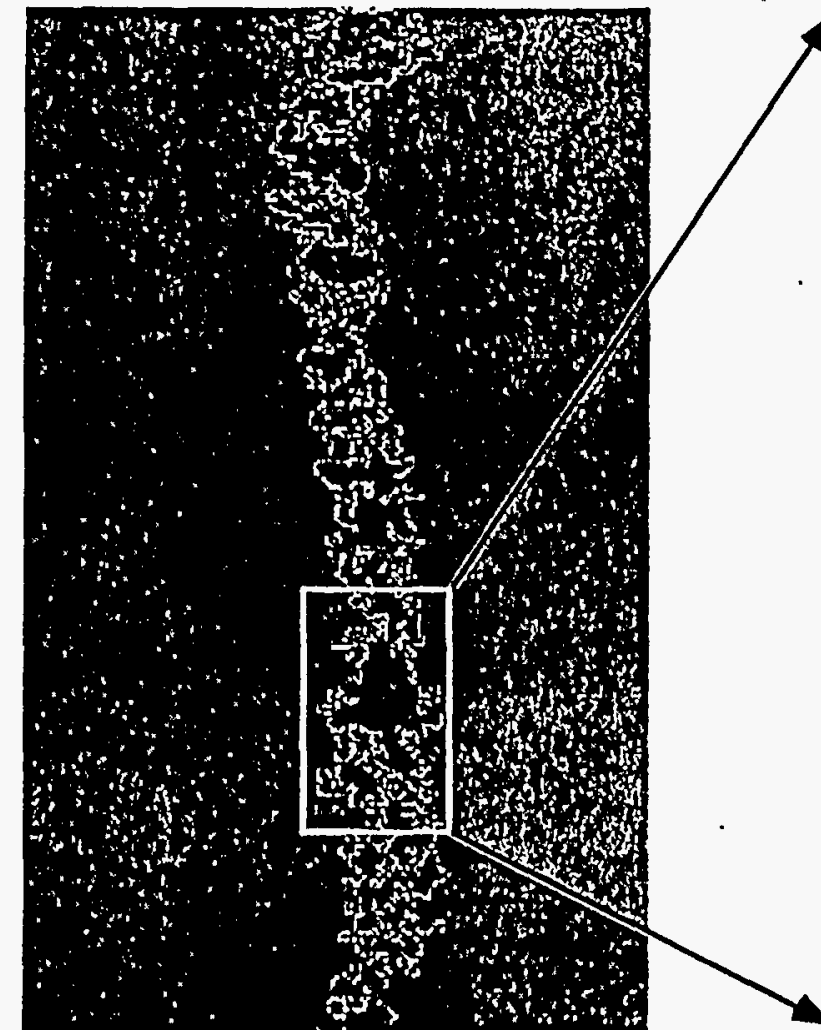

a)

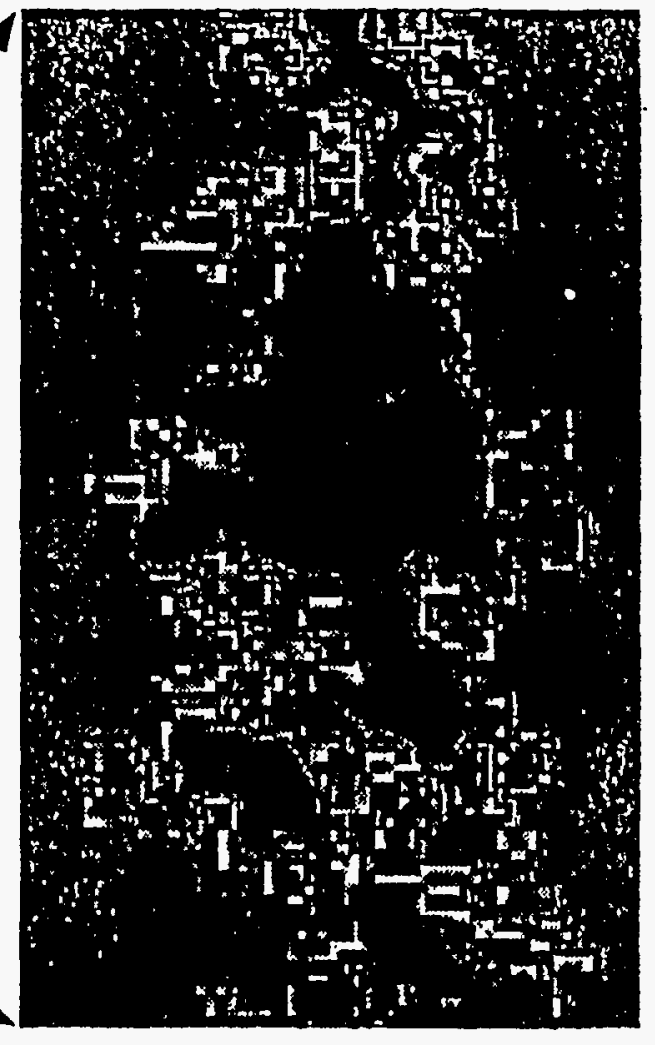

b)

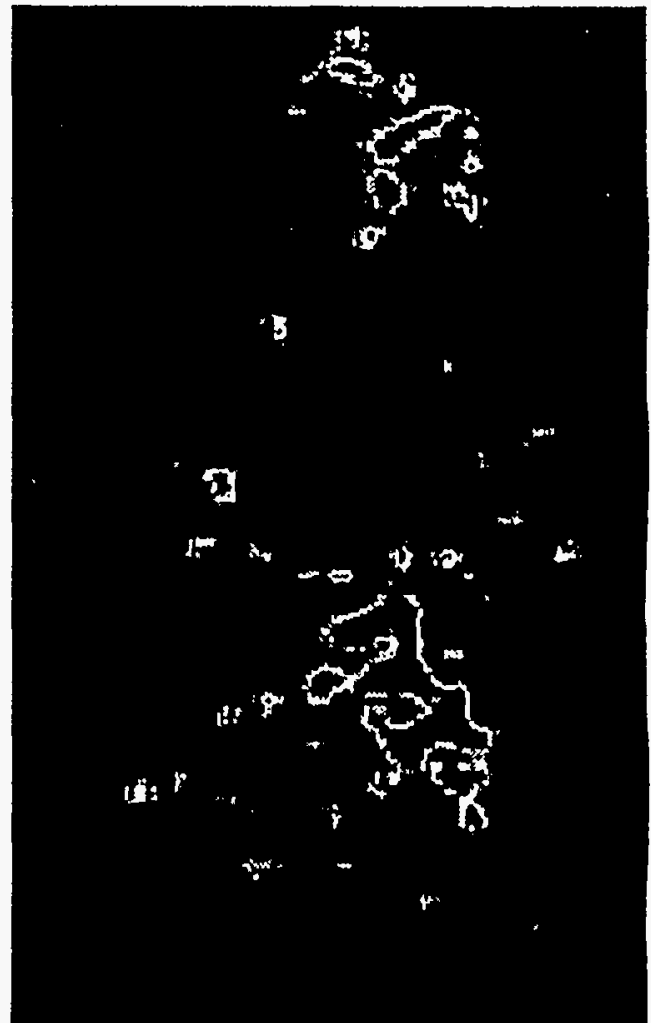

c)

Figure 23: Unsteady, and potentially chaotic, flow induced by steady supply to a vertical fracture: A statistically homogeneous (sec Figure 3) analog fracture $(15 \times 30 \mathrm{~cm})$ is used to explore dynamic behavior in the desaturated zone behind a finger-tip. A single finger is initiated in a dry, vertical fracture by steady flow $(0.025 \mathrm{ml} / \mathrm{minute}$ is shown here) to a point source on the upper boundary. Belind the finger-tip, a poorly connected desaturated zone develops $(a, b)$. Flow from the source to the finger-tip moves through a series of small, intermittently connected "reservoirs", shown as bluc/purple regions (a, b), red denotes dry, and jellow/green moist. Dynamic belavior of an individual reservoir (b) is explored by recording changes in saturation over time, as illustrated in c; hot colors (red, yellow) represent repeated drain and fill cycles (e.g., drip points), while cooler colors (blue, purple) indicate repealed expansion and coniraction of ihe reservoir, and black indicates no change. This behavior appears to be analogous to a series of coupled dripping faucets. Based on this observed behavior, the potential that system dynamics are chaotic is quite high (Nicholl, Glass, and Nguyen, 1993a). 
Dimensionless Finger Width :

$$
\frac{\text { finger width }}{\text { capillary length scale }}=\frac{W_{f}}{\Psi_{w}-\Psi_{a}}
$$

Dimensionless Flux:

$$
\text { (see caption) } \frac{Q}{K_{s} \cos \beta\left(\Psi_{w}-\Psi_{a}\right) \hat{a}}
$$

\section{where:}

$$
\begin{aligned}
K_{s} & =\text { saturated conductivity } \\
\beta & =\text { fracture inclination from vertical } \\
\cos \beta & =\text { gravitational gradient } \\
Q & =\text { volumetric flow rate } \\
\hat{a} & =\text { mean aperture } \\
\Psi_{w} & =\text { water - entry pressure } \\
\Psi_{a} & =\text { air - entry pressure } \\
W_{f} & =\text { measured finger width }
\end{aligned}
$$

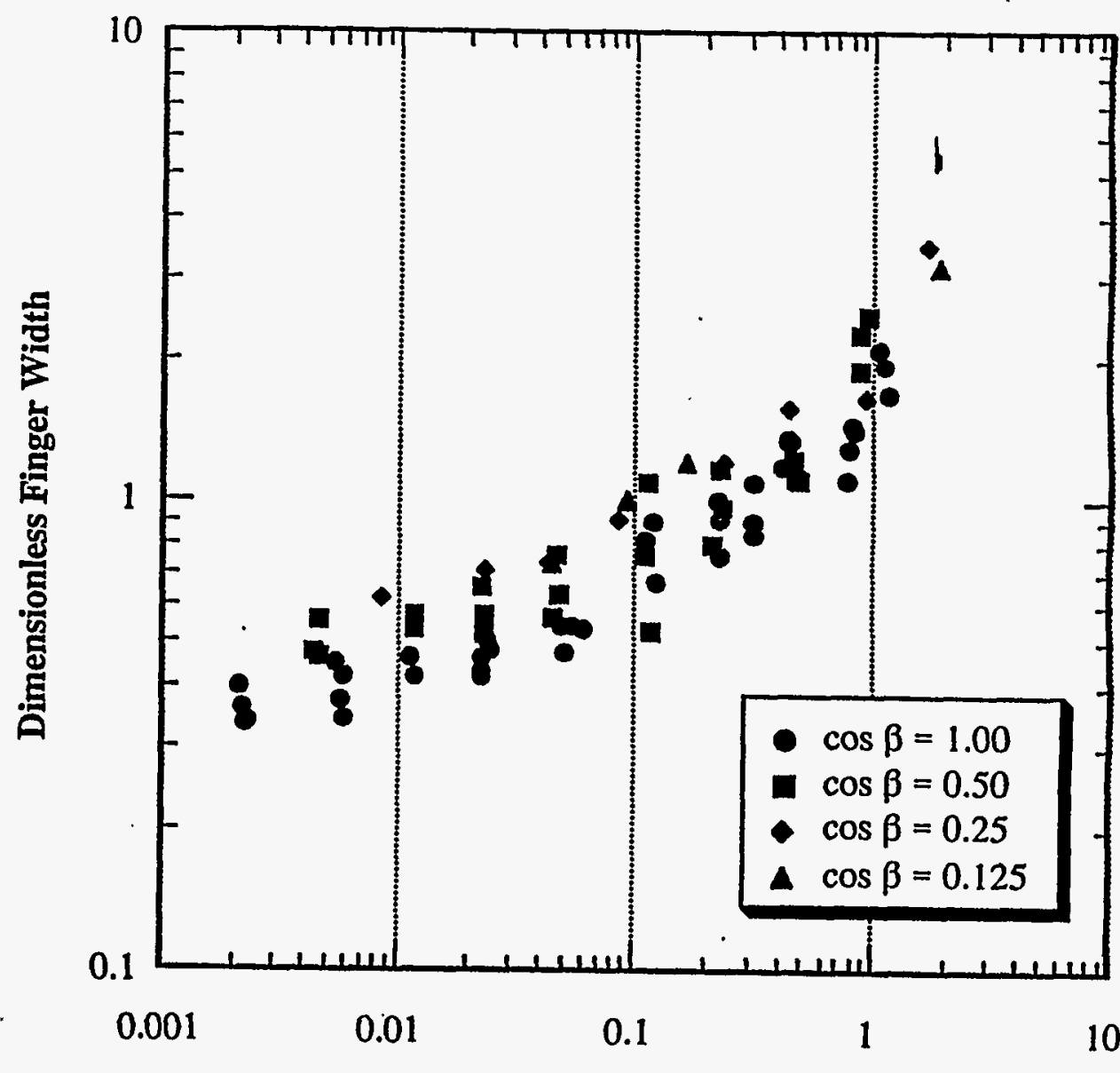

Dimensionless Flux

Figure 22: Finger width as a function of dimensionless flow rate: Experimental measurements of individual finger width in a statistically homogeneous (see Figure 3) analog fracture $(15 \times 30 \mathrm{~cm})$ are displayed in dimensionless form. Gravity-driven saturated flux through the analog fracture, defined as the product of saturated conductivity and gravitational gradient $\left(K_{S} \cos \beta\right)$ and the capillary length scale, defined as the difference between water-and air-entry pressures $\left(\psi_{w}-\Psi_{a}\right)$ are taken as characteristic measures of system hydraulic behavior; for dimensional correctness, the mean aperture (a) is also used to non-dimensionalize the input flow rate. Dimensionless finger width is observed to increase with dimensionless flow rate into the finger in a non-linear fashion. It can also be seen that this dimensionless form does not fully account for the effects of fracture inclination (b); the data plots on four distinct lines, one for each gravitational gradient $(\cos \beta$ ) considered, with finger width inversely proportional to $\beta$ (Nicholl, Glass, and Nguyen, 1993a). 
Dimensionless Finger Velocity:

$$
\frac{\text { measured finger velocity }}{\text { saturated flux }}=\frac{V_{f}}{K_{s} \cos \beta}
$$

\section{Dimensionless Finger flux:}

$$
\frac{\text { average finger flux }}{\text { saturated flux }}=\frac{Q}{W_{f} \hat{a}} \cdot \frac{1}{K_{s} \cos \beta}
$$

where:

$\beta=$ fracture inclination from vertical $\cos \beta=$ gravitational gradient

$Q=$ mass flow rate

$K_{s}=$ saturated hydraulic conductivity.

$V_{f}=$ finger velocity

$W_{f}=$ finger width

$\hat{a}=$ mean aperture

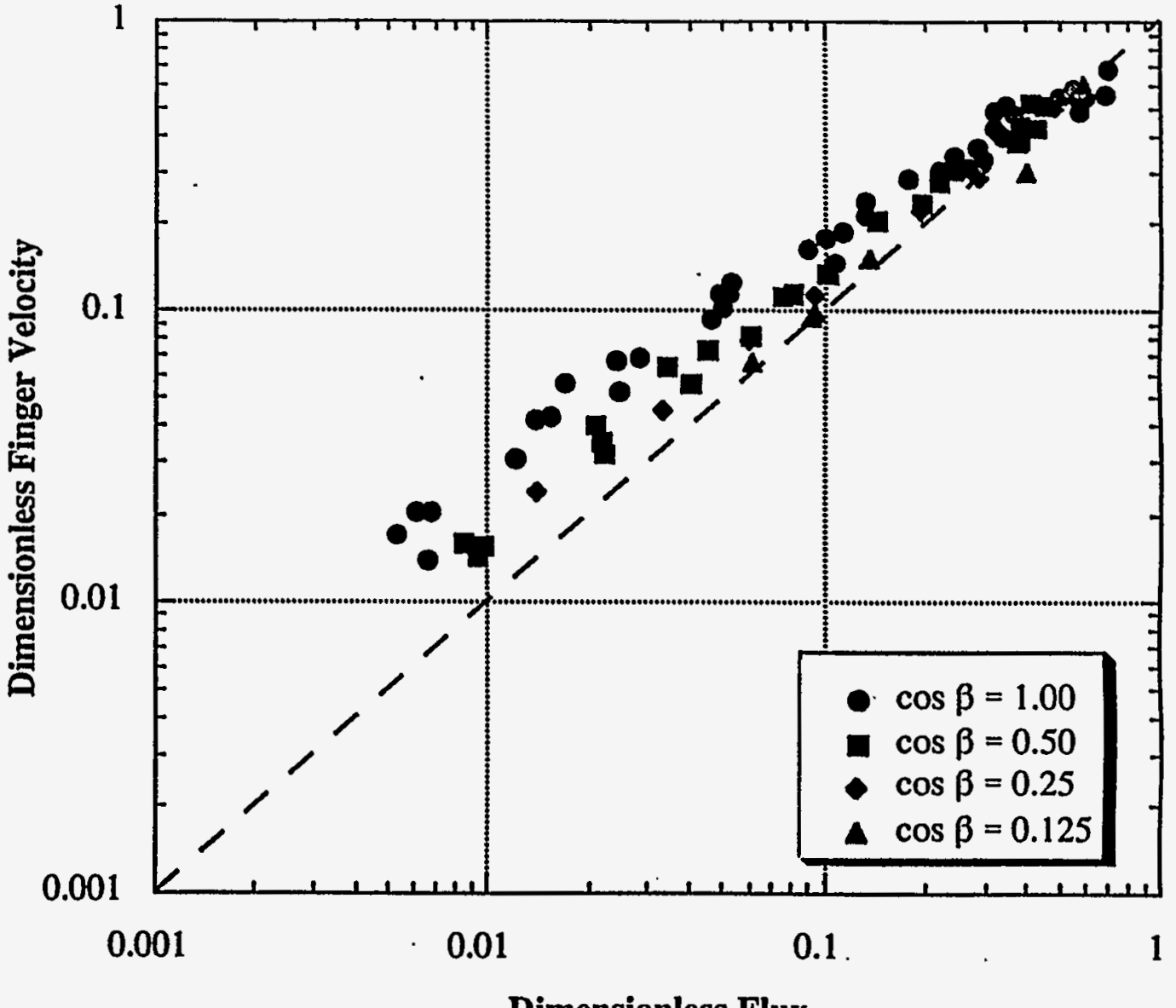

Figure 21: Dimensionless finger velocity as a function of dimensionless flux: Experimental measurements of individual finger velocity in a statistically homogeneous (see Figure 3), dry analog fracture $(15 \times 30 \mathrm{~cm})$ are displayed in dimensionless form. Gravity-driven saturated flux through the analog fracture, defined as the product of saturated conductivity and gravitational gradient (KS $\cos \beta$ ) is used to provide a characteristic measure of system hydraulic behavior. Dimensionless finger velocity is defined as the measured finger velocity relative to saturated flux. Dimensionless flux is defined as the average flux through the finger (input flow rate divided by the product of finger width and mean aperture) also scaled by the saturated flux. In the absence of desaturation behind the finger-tip, mass balance considerations would place all points on the dashed line (velocity $=$ flux). However, the existence of a desaturated zone allows the finger to advance faster than the applied flux (velocity $\approx$ flux/moisture content). The degree of observed desaturation increases with fracture inclination and is inversely proportion to applied flux; the ratio of measured velocity to applied flux is also observed to increase under those conditions (Nicholl, Glass, and Nguyen, 1993a). 

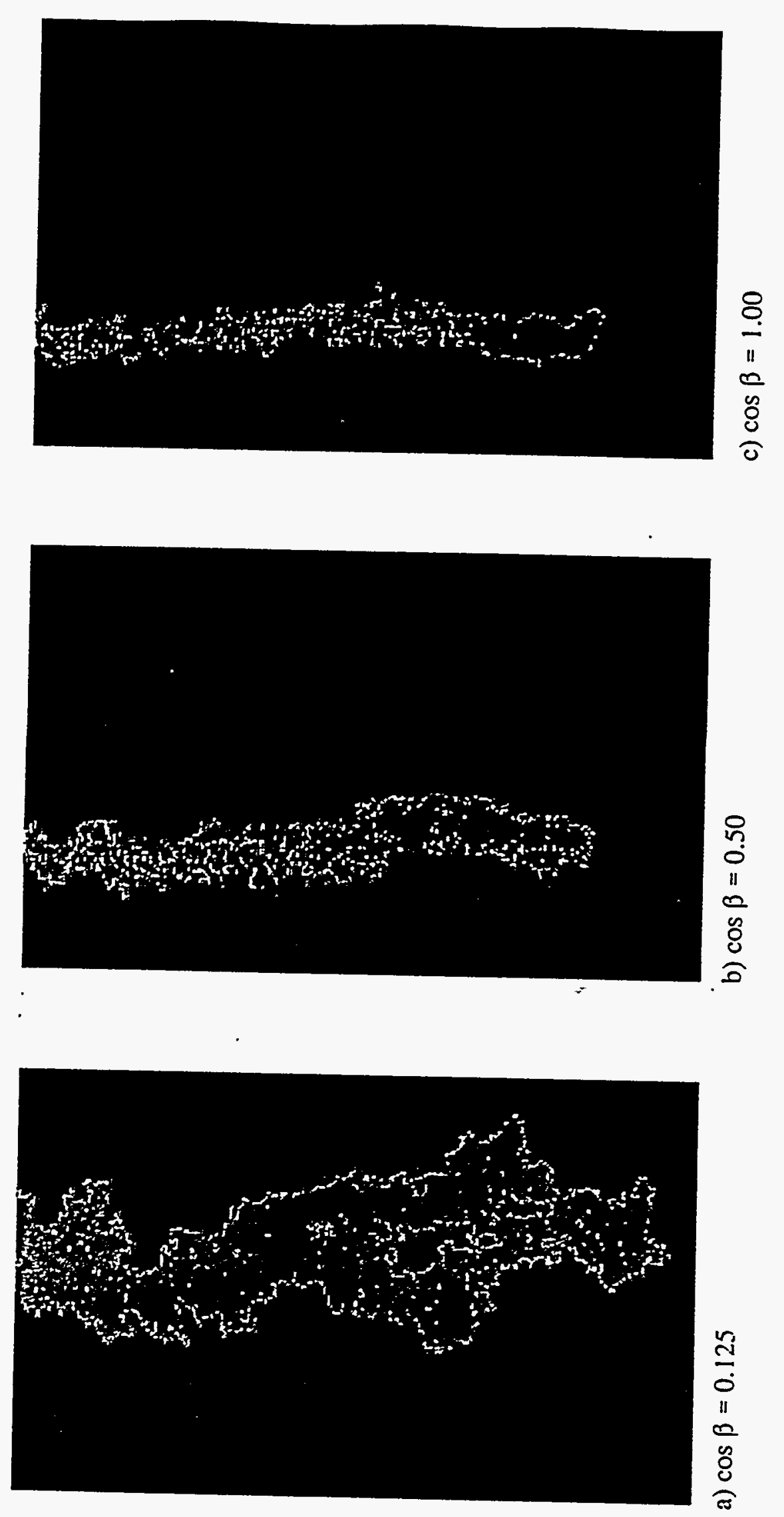

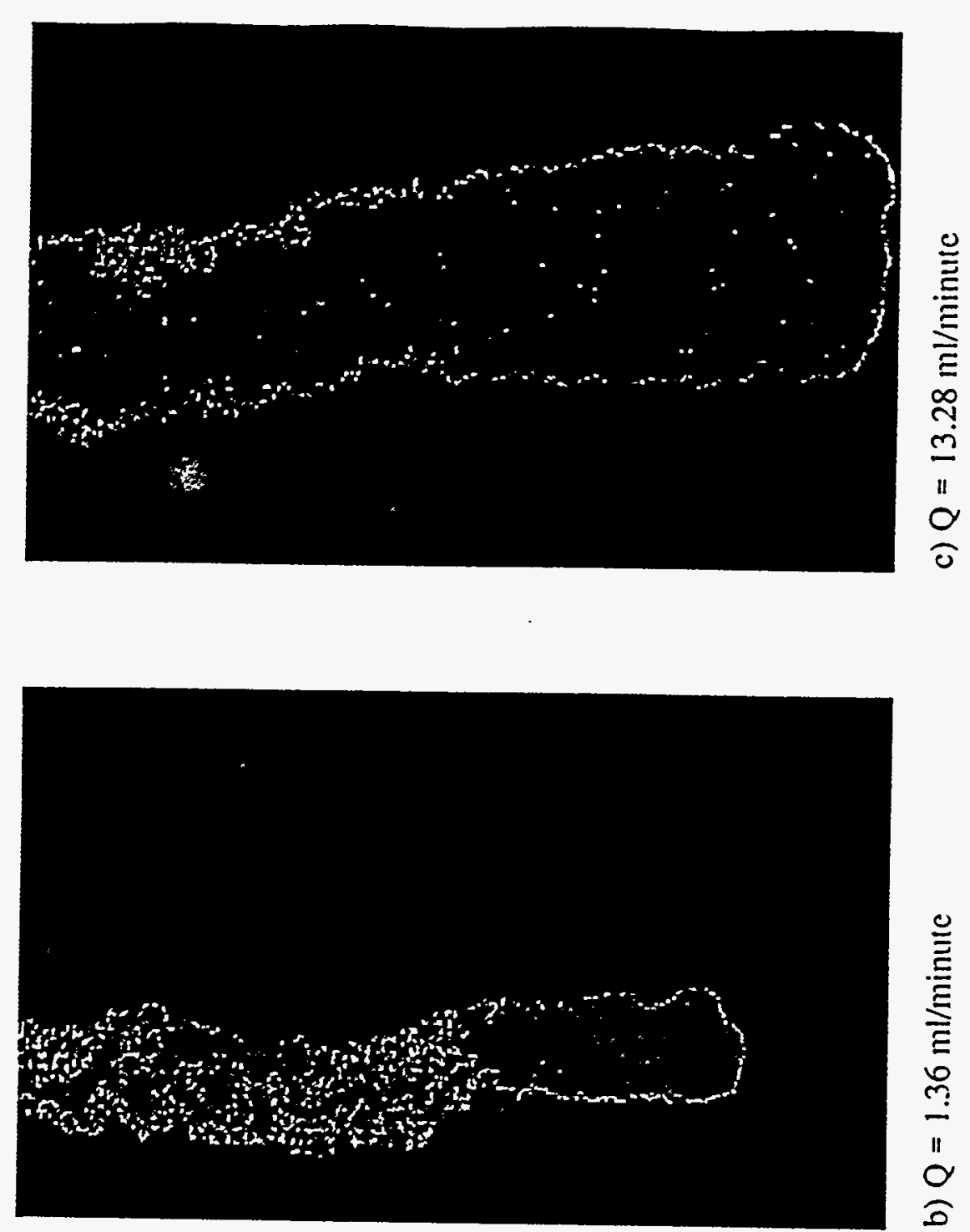

and

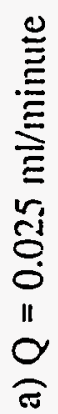

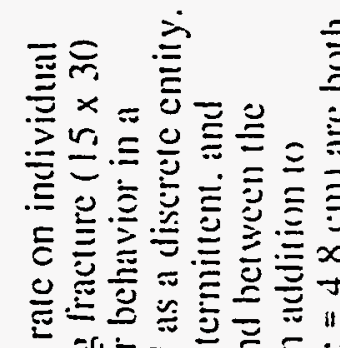
了行它可至三。 을

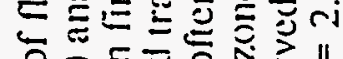

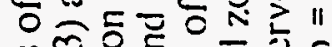
뜨을

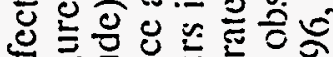
0

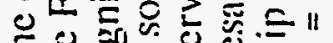

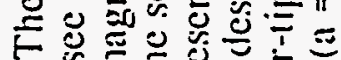
$\because 0 心 \Xi$

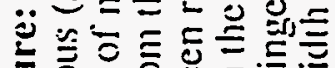

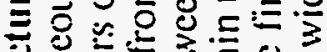

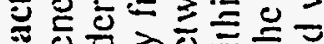

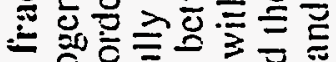
을

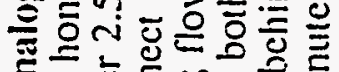

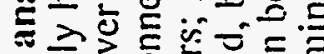

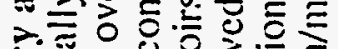

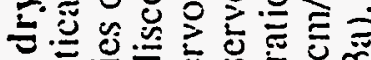

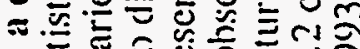

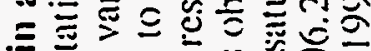

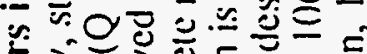

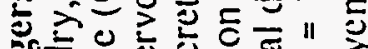

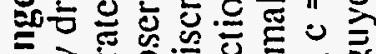

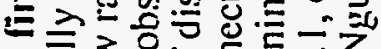

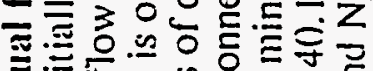

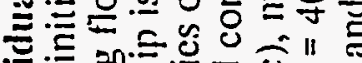
可

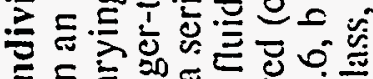
. . .

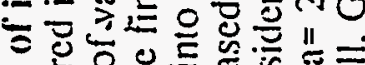

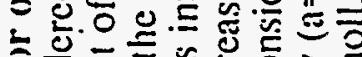

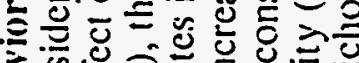

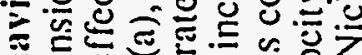

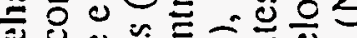
ڤญ

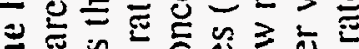

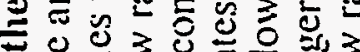

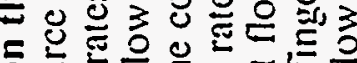

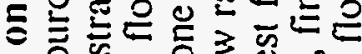

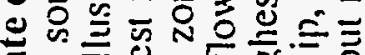

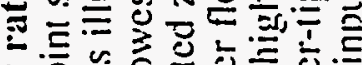

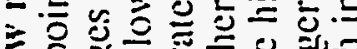

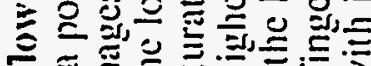

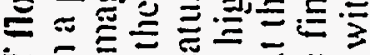

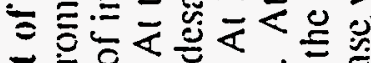

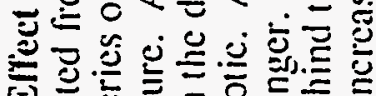

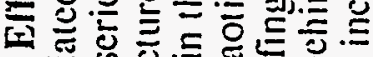

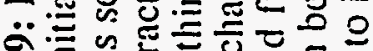

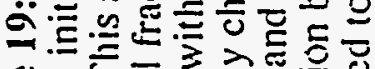

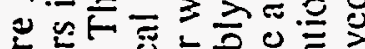

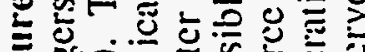

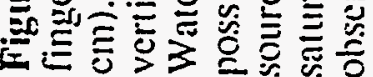




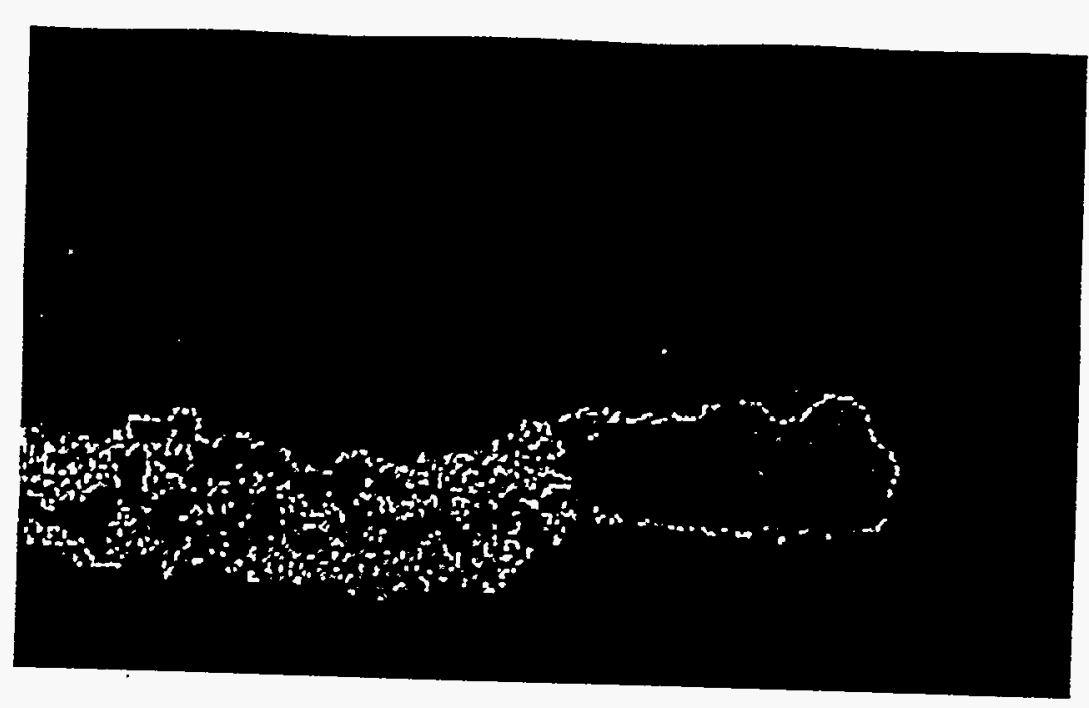

(

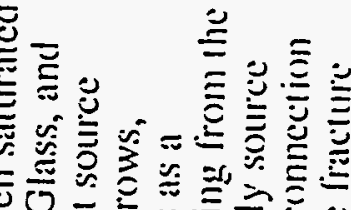

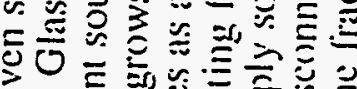

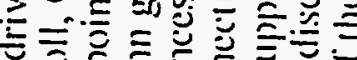

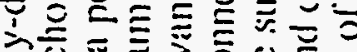

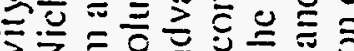

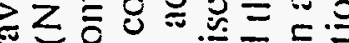

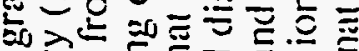

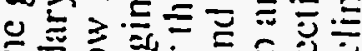

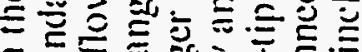
들

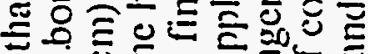

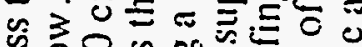

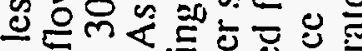

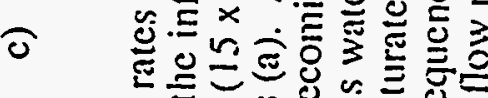

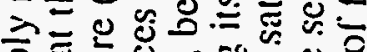

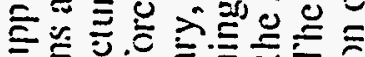

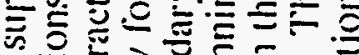
은

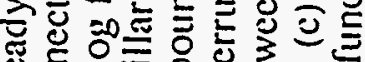

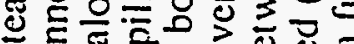

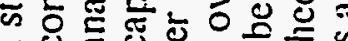

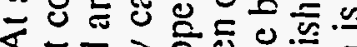

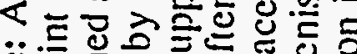

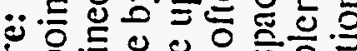
늘을

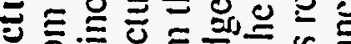

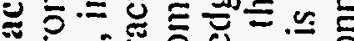

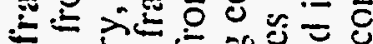

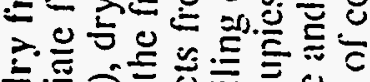

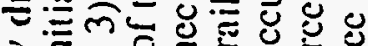
خ.E O OE

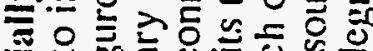
200

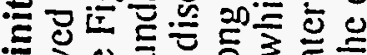

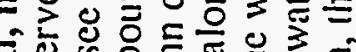

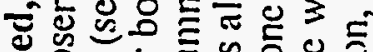

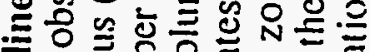

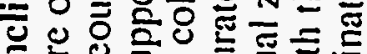

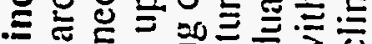
-

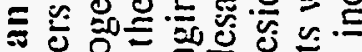

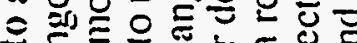

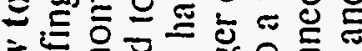
记可o

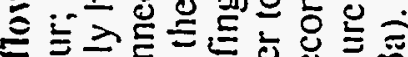

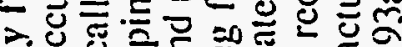

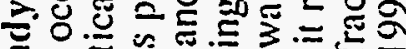

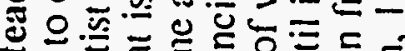

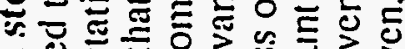
렁

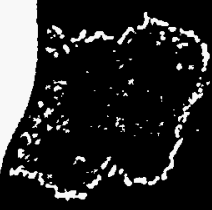

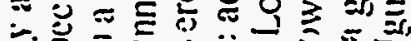

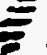

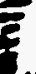

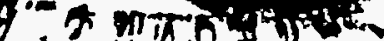
=

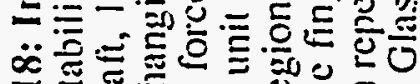

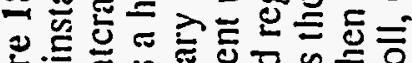
․․류

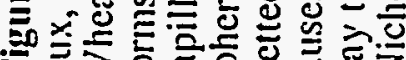

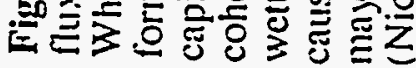


Flat-walled fracture (Hele-Shaw cell)

(1) $W=\pi\left\{\frac{3 \sigma L_{l}}{\rho g\left(\Psi_{w}-\Psi_{a}\right)}\right\}^{1 / 2}$

(after Chouke et al., 1959)

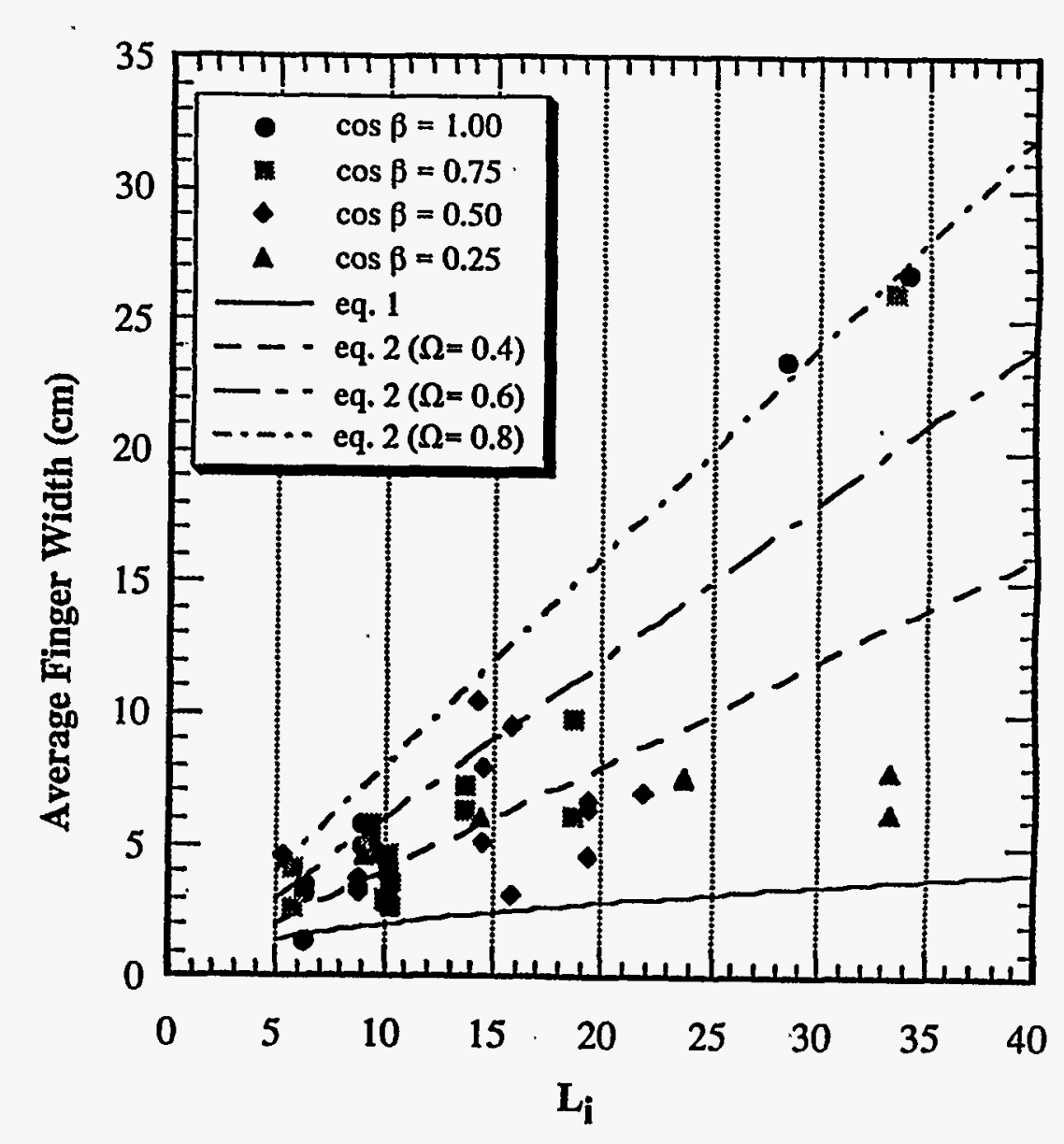

Two-dimensional porous media

$$
W=\Omega \bullet L_{i} \quad \text { (after Glass et al., 1991) }
$$

$$
\begin{aligned}
& \text { where: } \\
& \Omega=\frac{2 \pi \Gamma}{K_{s}\left(\Psi_{w}-\Psi_{a}\right)} \\
& K_{s}=\text { saturated hydraulic conductivity } \\
& \Gamma=\mathrm{f} \text { (moisture content, sorptivity) see Glass et al., } 1991
\end{aligned}
$$

Figure 17: Comparison between measured finger width and linear stability theory: Gravity-driven fingers initiated at the cessation of ponded infiltration into an initially dry, statistically homogeneous (see Figure 3) analog fracture are compared to theoretical results for Hele-Shaw cells and 2-D porous media. Neither model fits the data well, although the Hele-Shaw model may provide an approximate lower bound. The experimental fingers initiated from finite-amplitude perturbations to the air-water interface, which is inconsistent with the assumption of infinitesimal perturbations required by linear stability theory (Nicholl, Glass, and Wheatcraft, 1994). 


\section{Model Assumptions:}

- Assume Darcy's Law is applicable

- Define dimensionless finger velocity $\left(\frac{V}{K_{s} \cos \beta}\right)$

- Assume air- and water-entry pressure heads are independent of velocity

Model:

$$
\frac{V}{K_{s} \cos \beta}=\left\{1-\frac{\left(\psi_{w}-\Psi_{a}\right)}{L_{f} \cos \beta}\right\}
$$

$$
\begin{aligned}
& \text { where: } \\
& V=\text { finger velocity } \\
& \mathrm{K}_{\mathrm{s}}=\text { saturated hydraulic conductivity } \\
& \beta=\text { fracture inclination from vertical } \\
& \cos \beta=\text { gravitational gradient } \\
& \mathrm{L}_{\mathrm{f}}=\text { finger - tip length } \\
& \psi_{\mathrm{a}}=\text { air - entry pressure head } \\
& \psi_{\mathrm{w}}=\text { water - entry pressure head }
\end{aligned}
$$

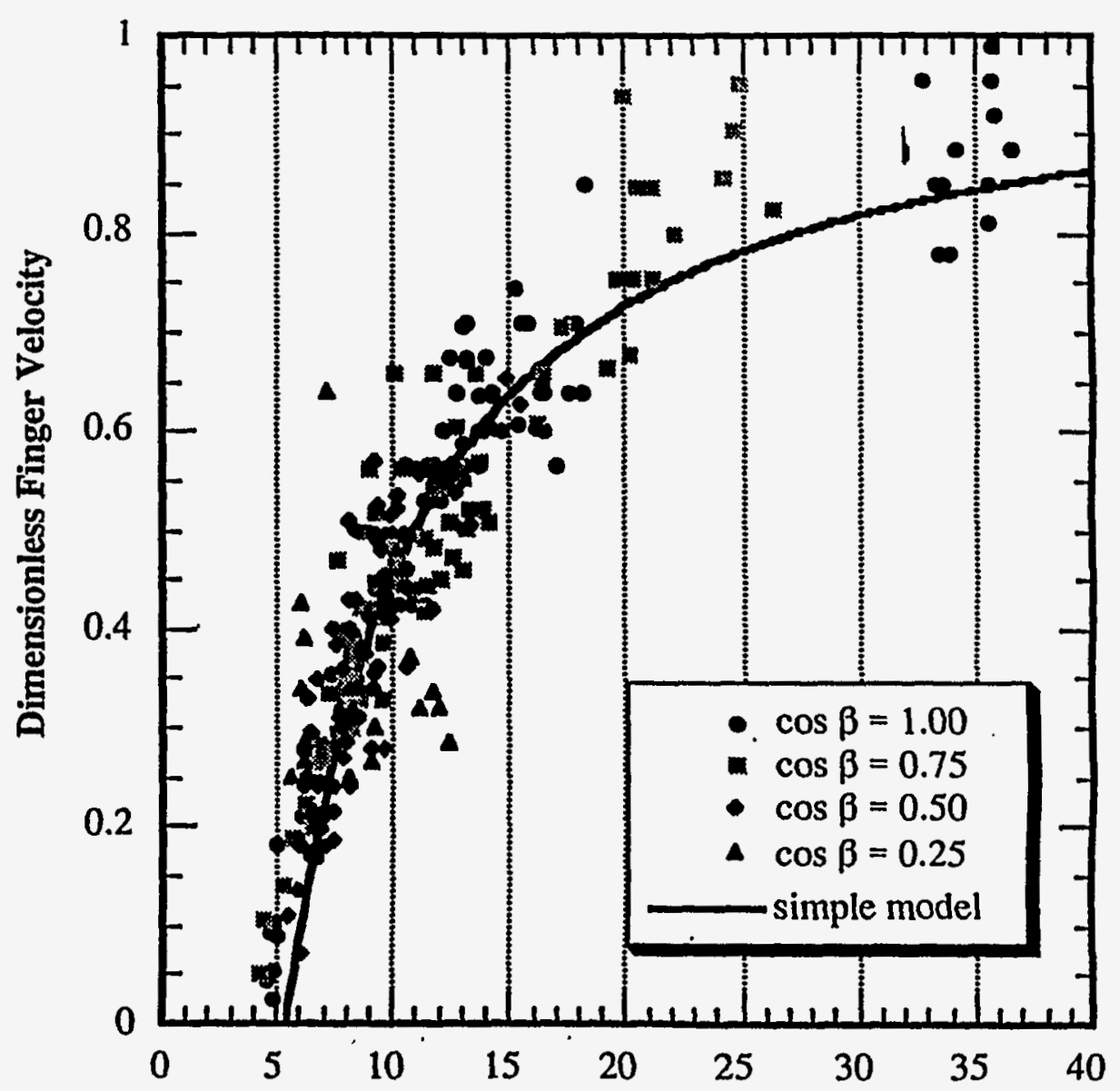

Finger-tip Length (cm)

Figure 16: Finger velocity as a function of finger-tip length: Fingers developed from redistribution following ponded infiltration travel as isolated, coherent units (finger-tips). The length of these finger-tips along the gravitational vector provides a measure of the capillary gradient opposing flow. A first-order model relating finger-tip velocity to finger-tip length is developed by assuming that Darcy's Law is applicable; the bulk of the finger-tip is moving under saturated conditions; and that pressures along the leading and trailing edges of the finger-tip are adequately represented by the water- and air-entry pressures, respectively (Nicholl, Glass, and Wheatcraft, 1994). 


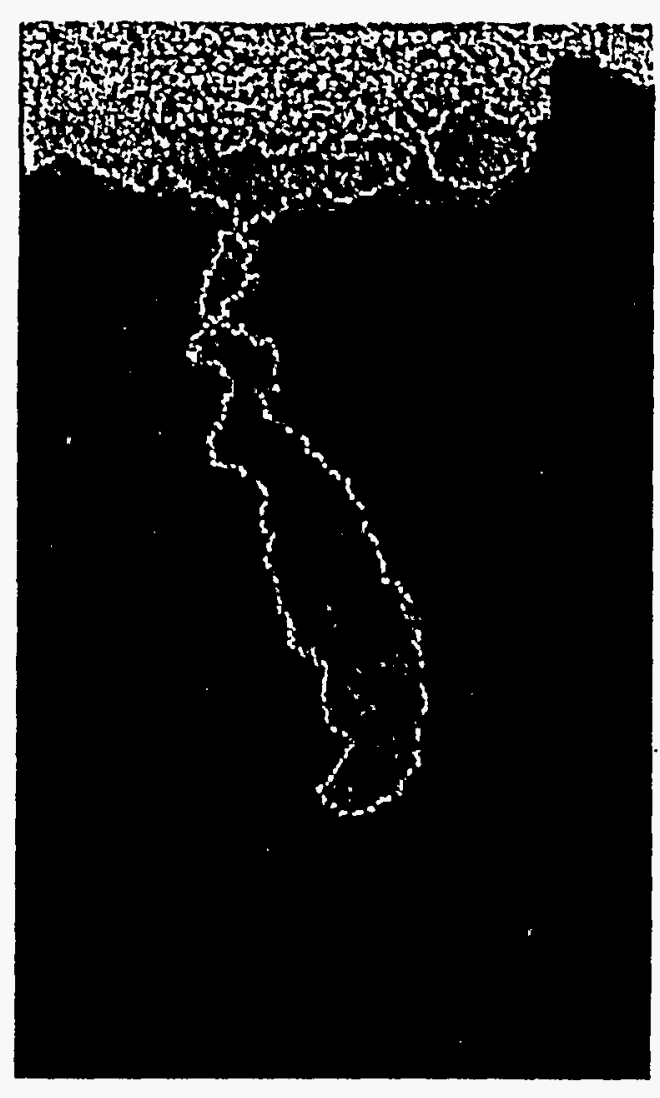

a) $\cos \beta=0.25, \mathrm{t}=1518$ seconds

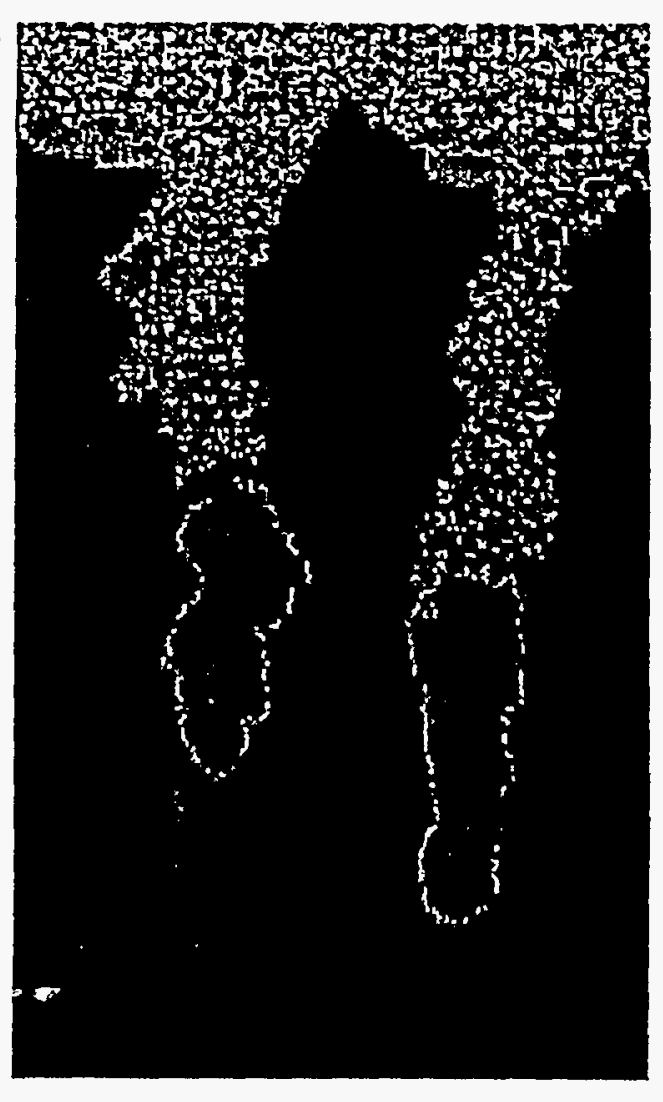

b) $\cos \beta=0.50, t=104$ seconds

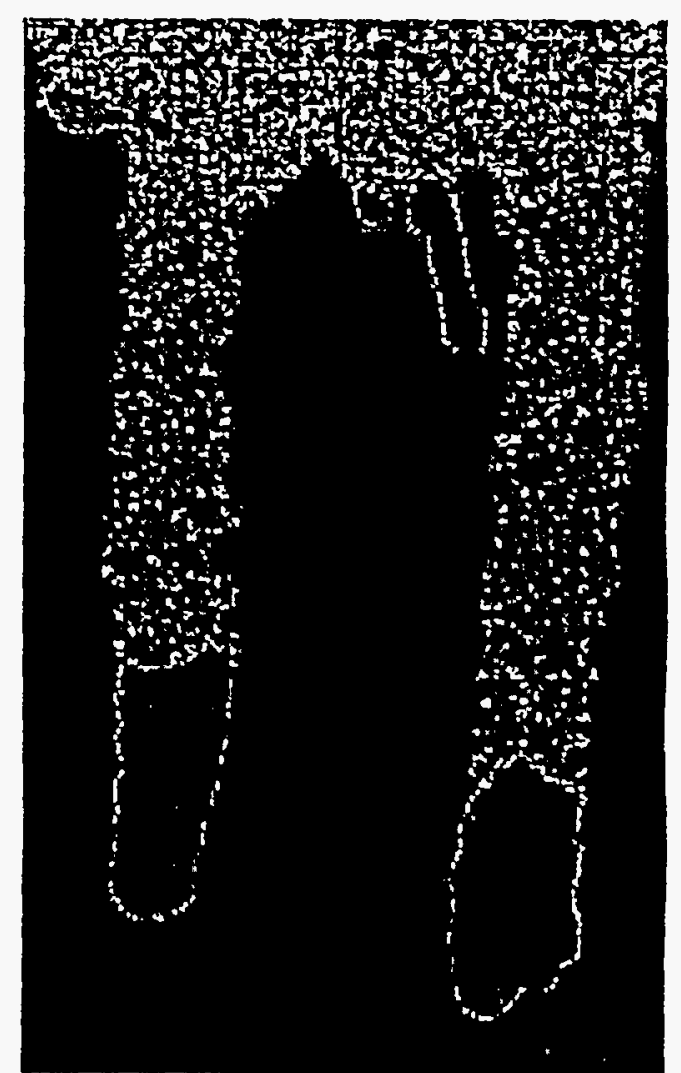

c) $\cos \beta=0.75,1=31$ seconds

Figure 15: Effects of gravitational gradient $(\cos \beta)$ on fingers formed from ponded infiltration: Ponded infiltration is simulated in an initially dry, statistically homogeneous (see Figure 3 ) analog fracture $(30 \times 60 \mathrm{~cm}$ ) by rapidly applying a finite slug of water 10 the upper boundary of the fracture; instability occurs when the slug is exhausted. Gravity effects on finger development are considered hy comparing experiments performed at various inclinations and similar input volume $(6.3 \mathrm{ml} \pm 5 \%)$. Al near horizontal inclinations $(\beta=$ 75.5 degrees, $\cos \beta=0.25$ ), observed behavior varies significantly in response to changes in the length of the waller column, or finger (a). At small lengths, gravity and capillary forces are near equilibrium; water advancement occurs in a series of individual jumps that initiate from the largest perturbation. As the finger lengthens, it's advancement velocity and width also increase, forming the finger seen here 1518 seconds after filling of the pond. At stecper inclinations, $(\beta=60$ degrees, cos $\beta=0.50)$, fingers are observed to develop) rapidly and advance as coherent units, forming the wetted structure shown (b) in 104 seconds. (c) As inclination increases $(\beta=41.4$ degrees, coss $\beta=$ (0.75), the dominance of gravity over capillary forces also increases, resulling in greater finger velocity ( 31 secondis) and less walndering. At a vertical inclination (not shown), 23 seconds was recuired to cover a distance similar to the other images shown here (Niclesll, (ilass, and Whealcraft, 1994). 

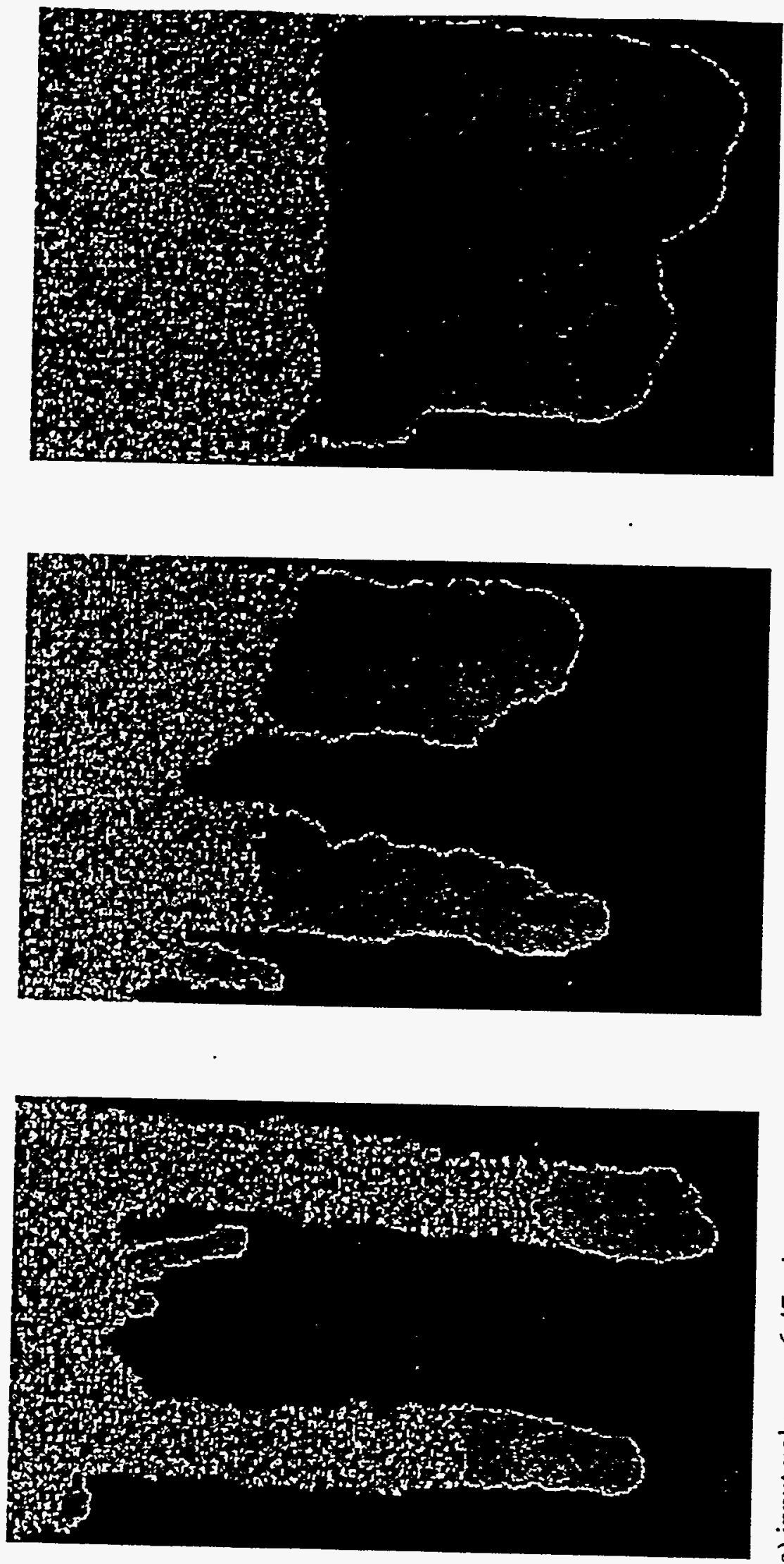

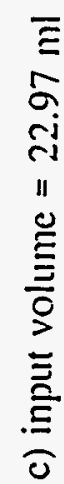

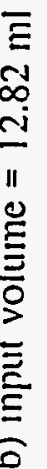

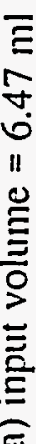

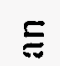

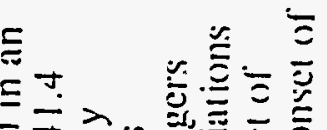

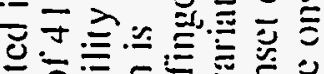

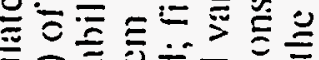

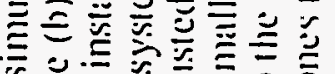

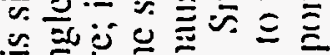
$\bar{\Xi} \equiv \overline{\bar{x}} \dot{\bar{\vdots}} \overline{\bar{x}}$

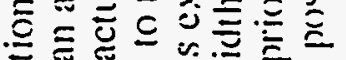

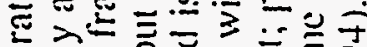

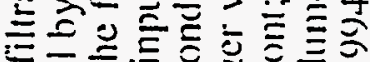

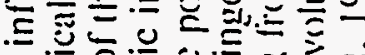

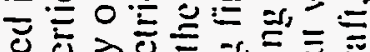

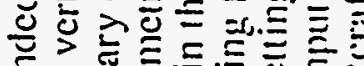

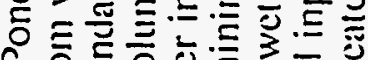

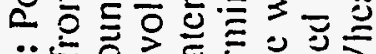

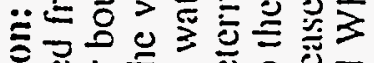

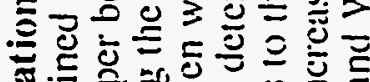

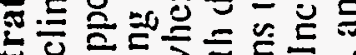
흘.

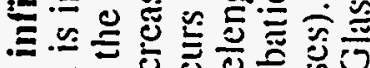

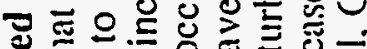

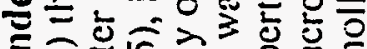
츠음

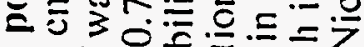

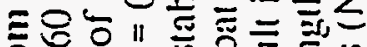

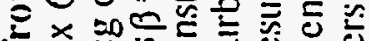

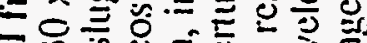

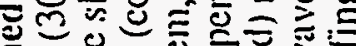
을 온르응

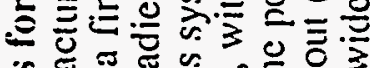
\% $\approx \pi$.

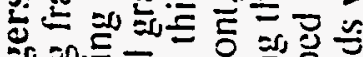

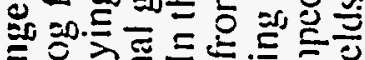

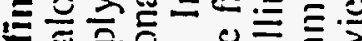
=

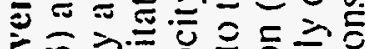

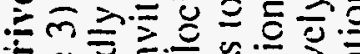

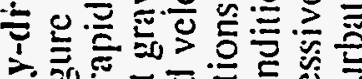

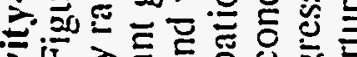

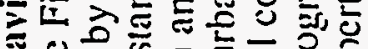

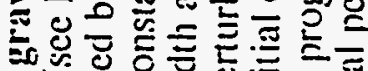

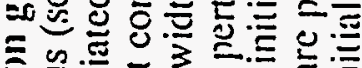

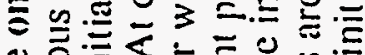

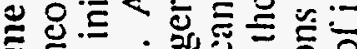

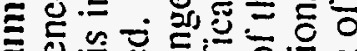

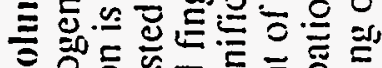

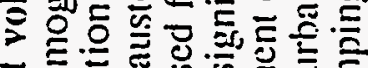

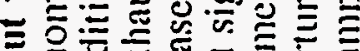

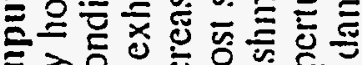

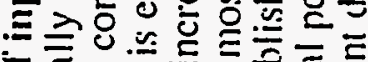

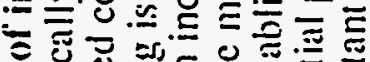

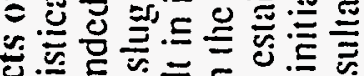
包.

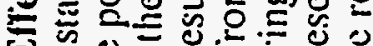
그은

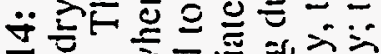

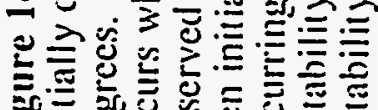

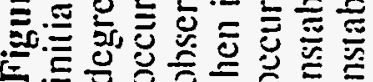



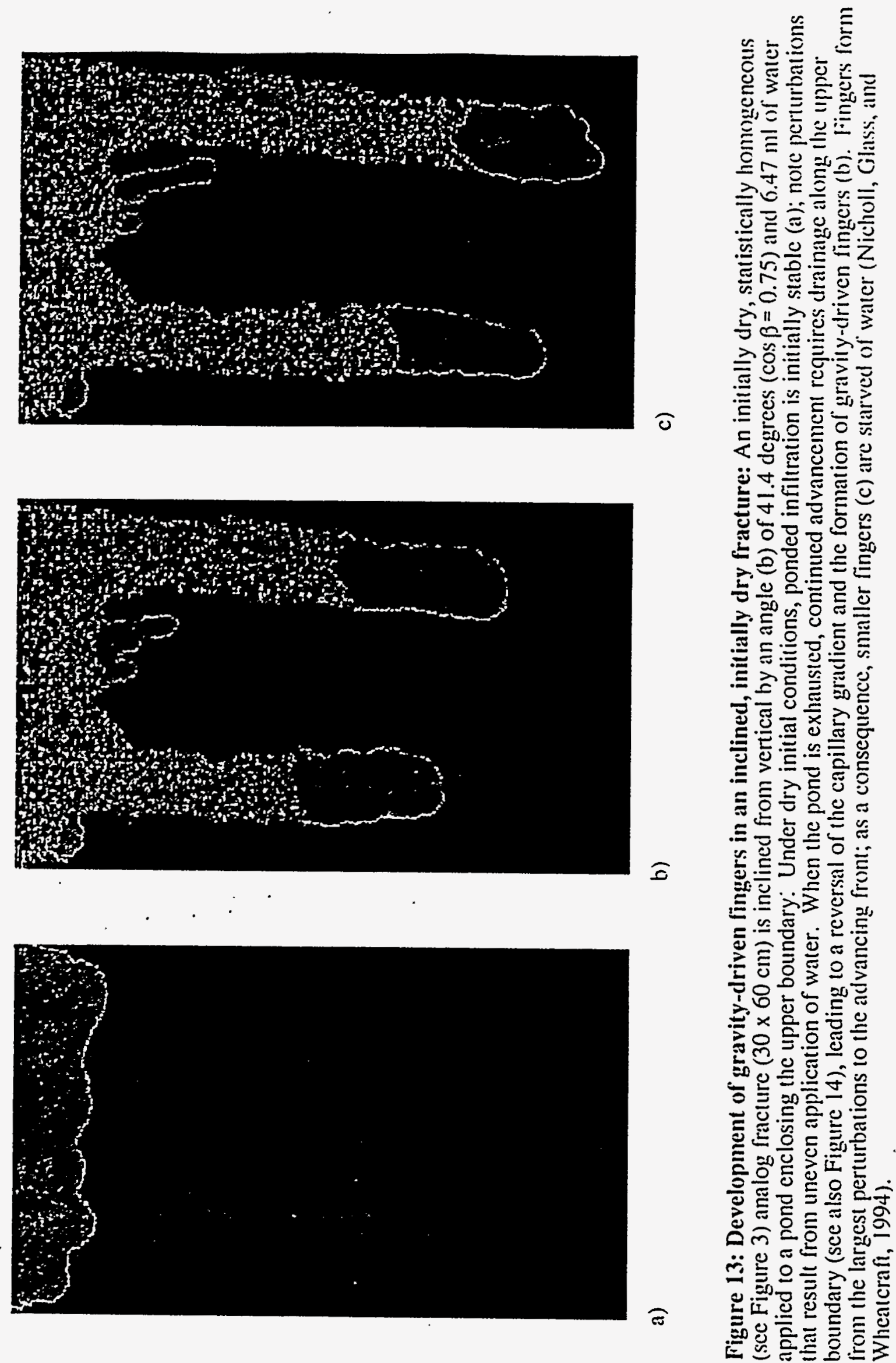

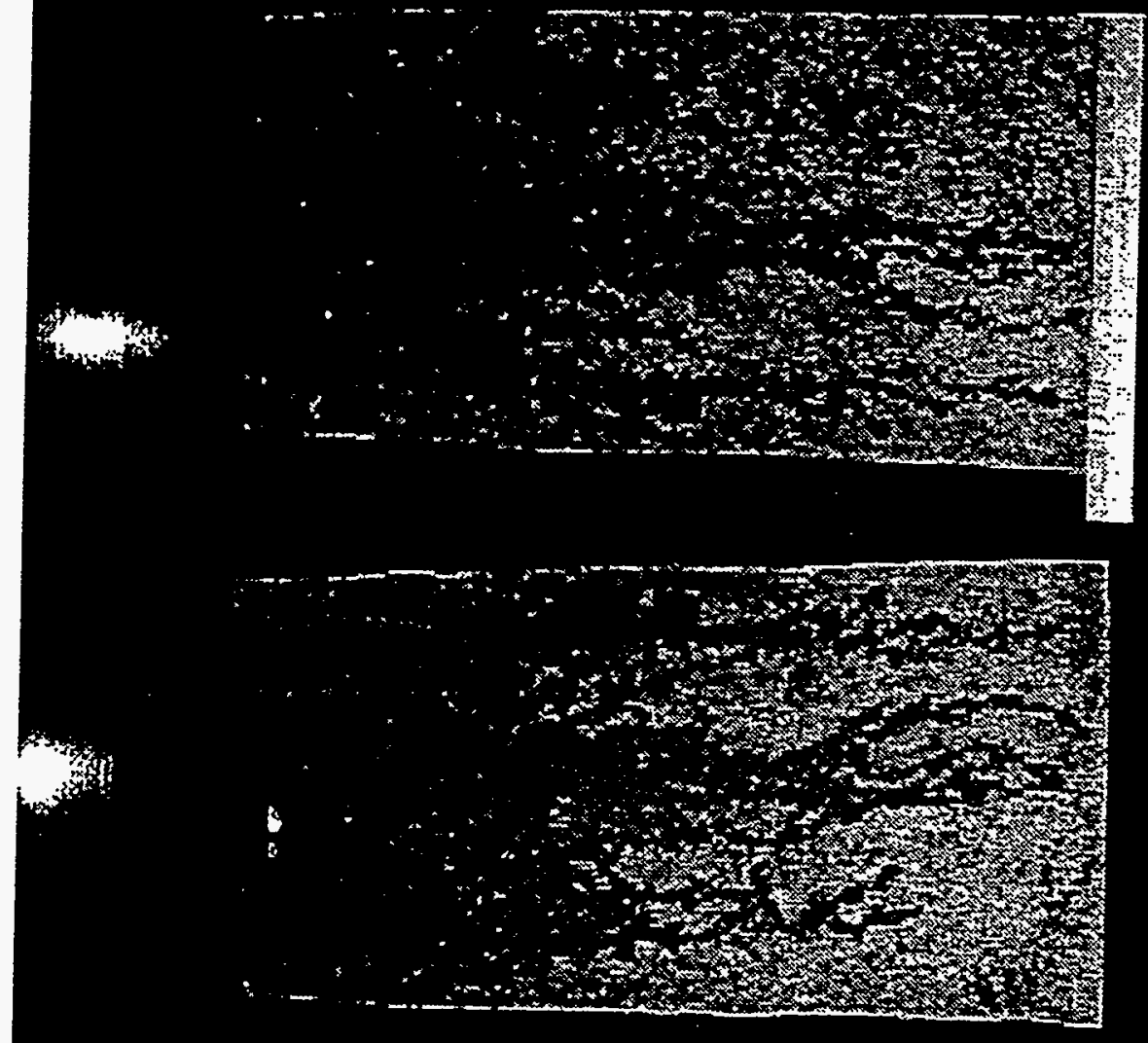

s" 든잉 20

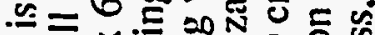
즐

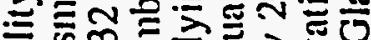
희

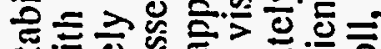

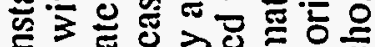
뜬

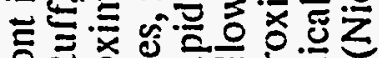

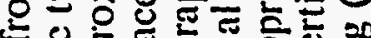

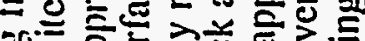

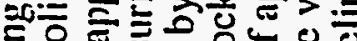

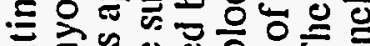
츤. 은

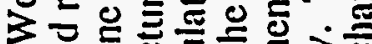
驱 ह

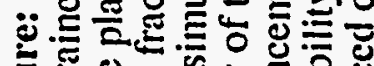

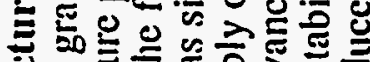
일 는

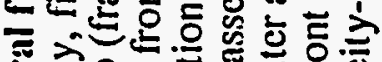

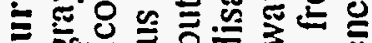
50.

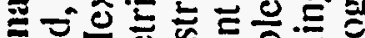

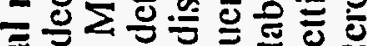
ปัँ

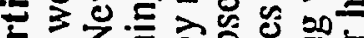

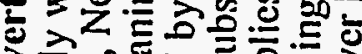

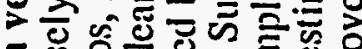
$\checkmark$ 论 $\equiv \overline{0} \equiv$

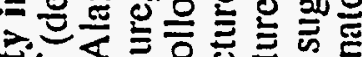
히는

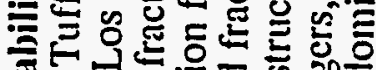
施可过

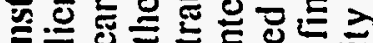
. 응 200. 층.․ㅡ잋 혼 - 可 ผ

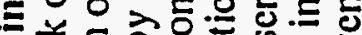

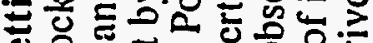

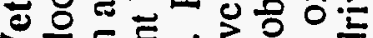

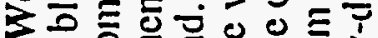

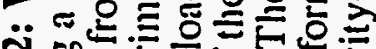

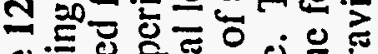
0.

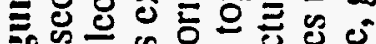

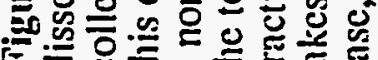




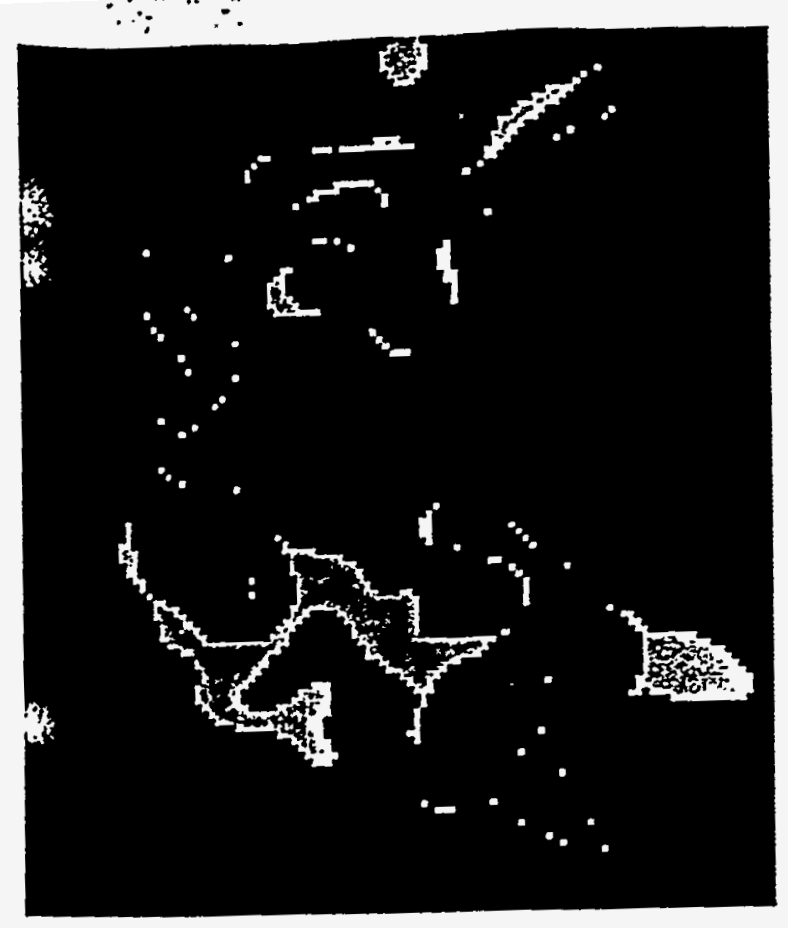

a)

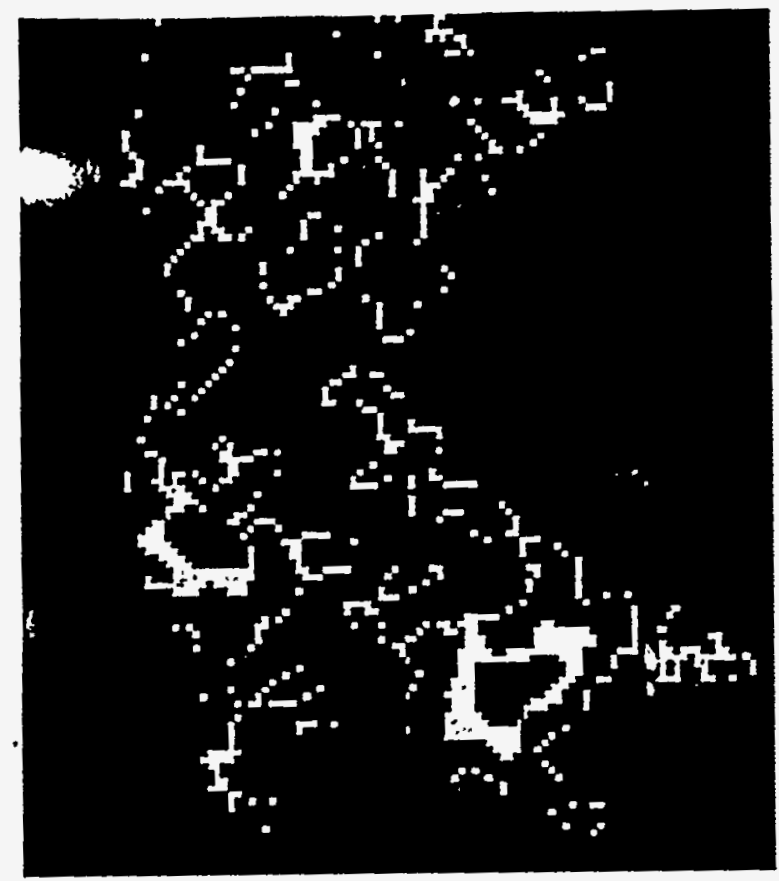

c)

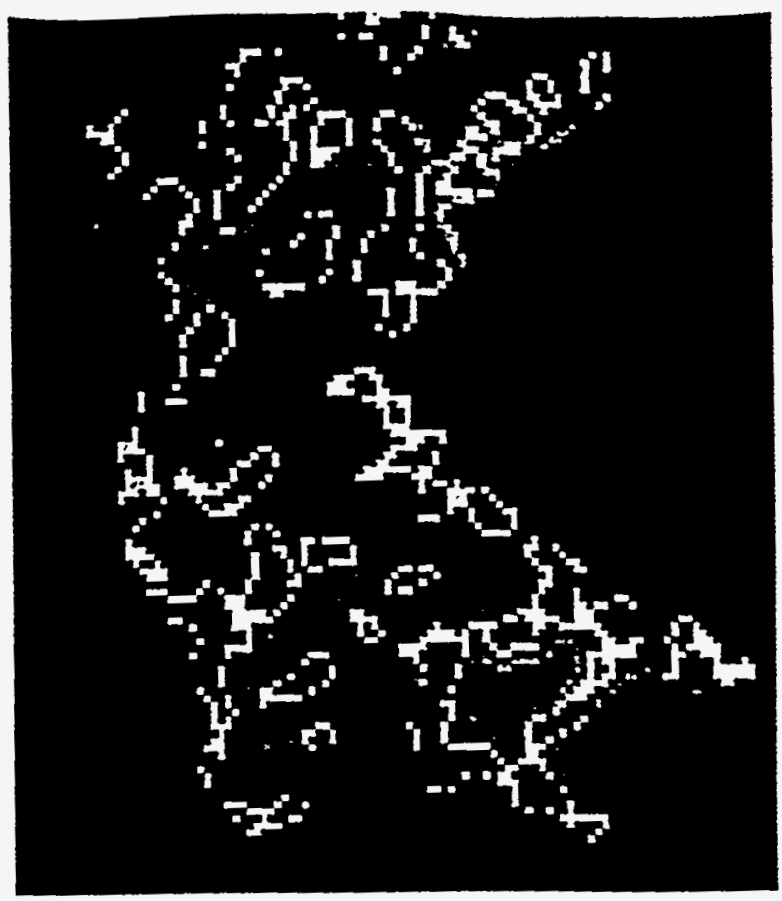

b)

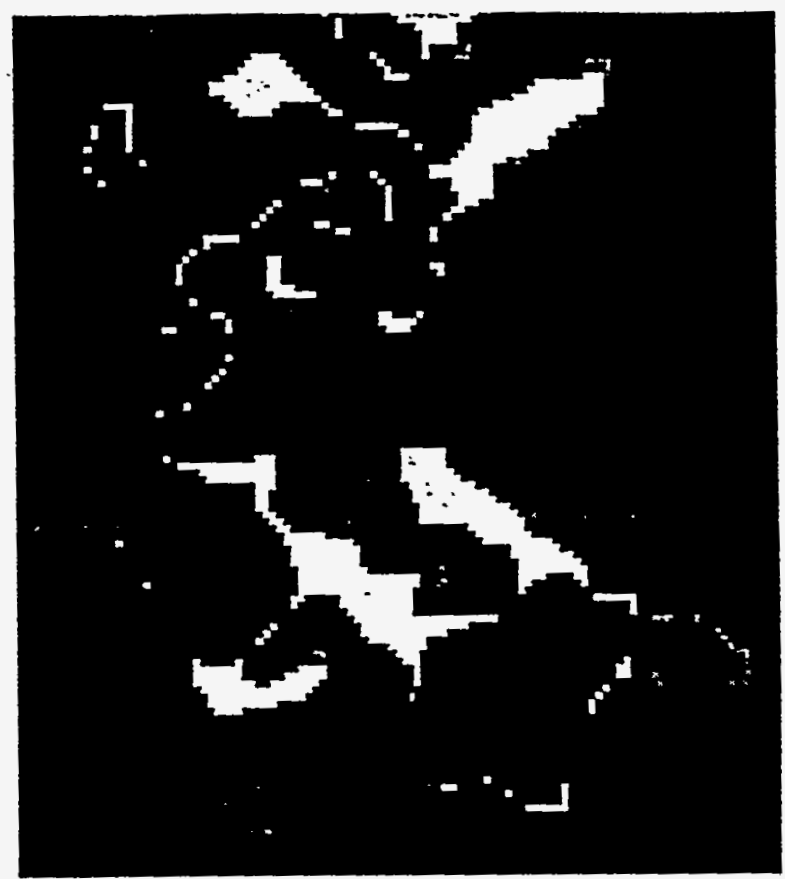

d)

Figure 11: Comparison of observed dissolution to standard percolation, standard invasion percolation, and modified invasion percolation models: In a $30 \times 15 \mathrm{~cm}$ horizontal analog fracture, deaerated water is used to dissolve the stationary air phase. Observations of dissolutioninduced replacement of the air phase (a) are then compared to predictions by various implementations of percolation theory (b-d); for illustrative purposes, a single air-filled cluster occupying a $1.95 \times 1.77 \mathrm{~cm}$ section of the aperture field is shown. Numerical simulations are run using the experimental aperture field measured at a spatial resolution of $0.15 \mathrm{~mm}$. Regions where no change in phase saturation occurs are displayed as black (water) and red (air). The other colors illustrate the order of dissolution, as follows: violet-blue-green-yellow. The physical experiment (a) differs significantly from both standard percolation (b) and standard invasion percolation (c). Modification of invasion percolation to include in-plane interfacial curvature (e.g., Glass, 1993) provides greatly imp:oved results (d) (Glass and Nicholl, 1995). 


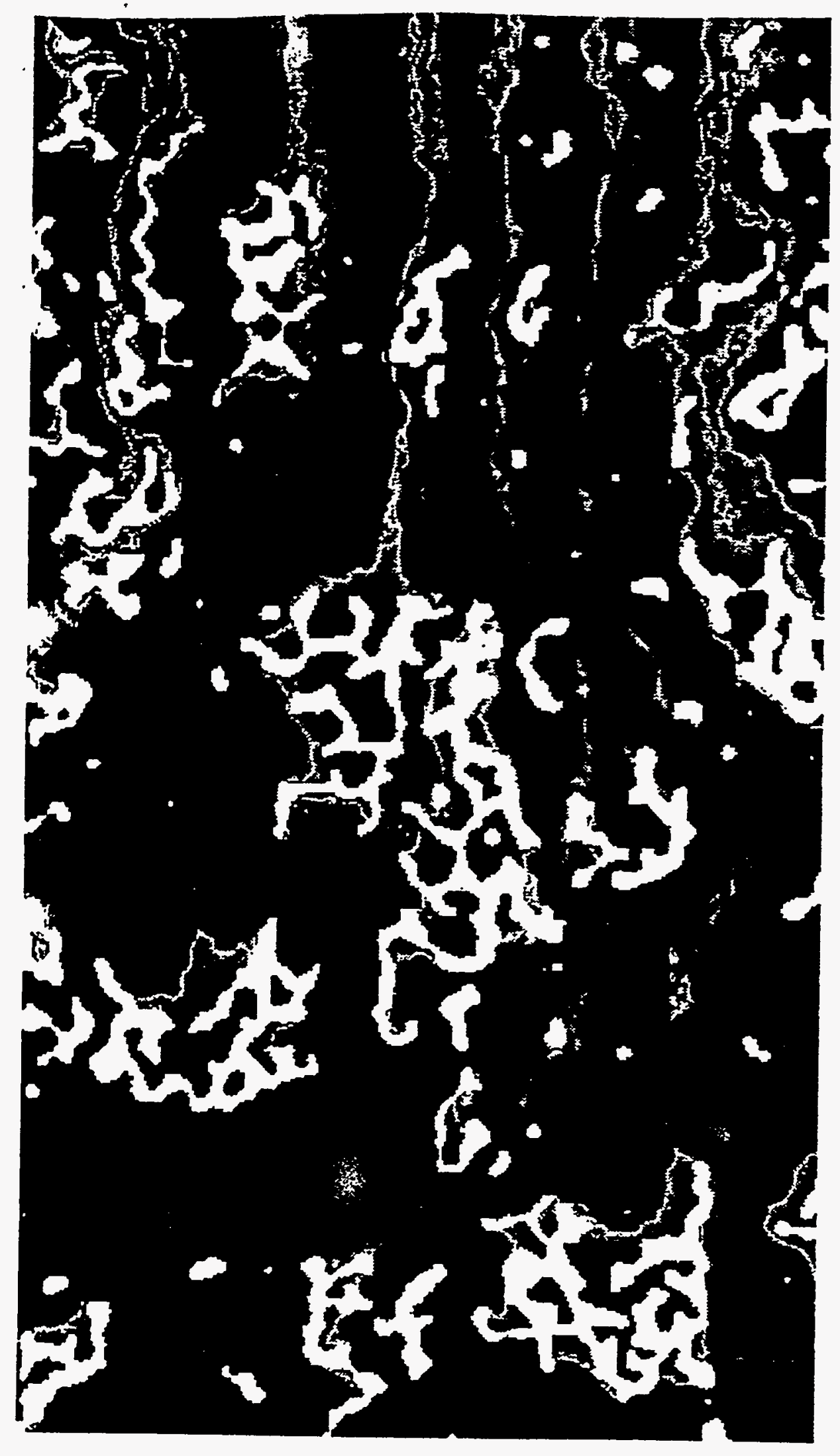

ธั

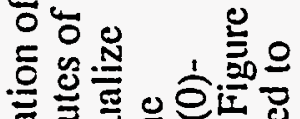

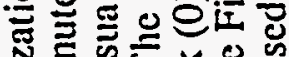

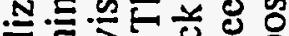
O

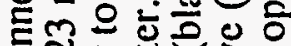

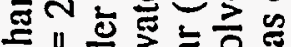
에을

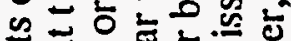

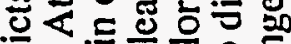
엉둥잉

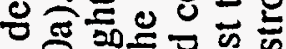
으는

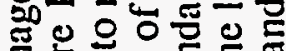

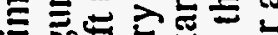
里可的可

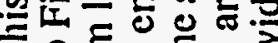
F。이용 른현을 응 은 뭉

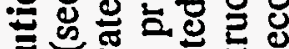
구은

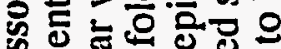

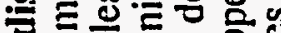
의

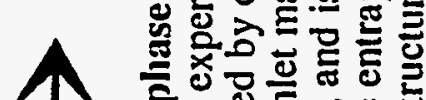
덩. Ј 응 을 흥 달

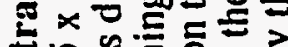

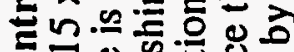

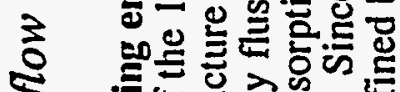

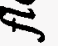

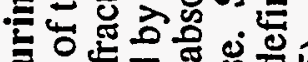

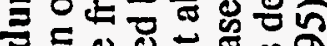

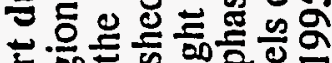
는

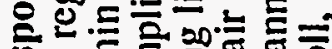

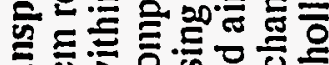
Tల

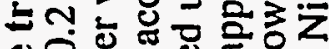
은은 인 된든

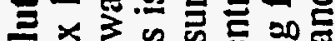
我政

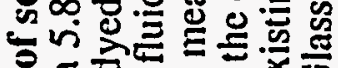
ป

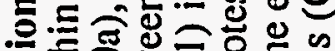
드은 추웜웡 든잉 플

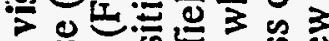

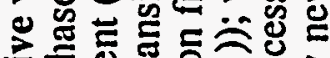

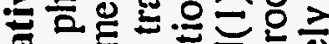

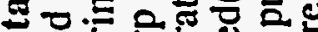

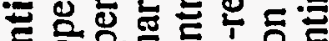
드의.

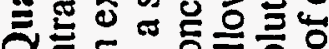

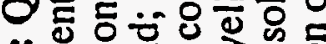

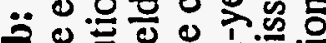

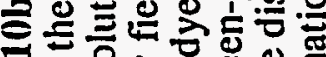

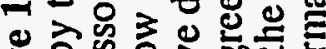
근은.

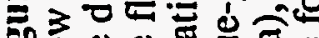

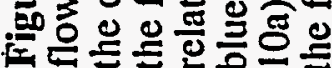




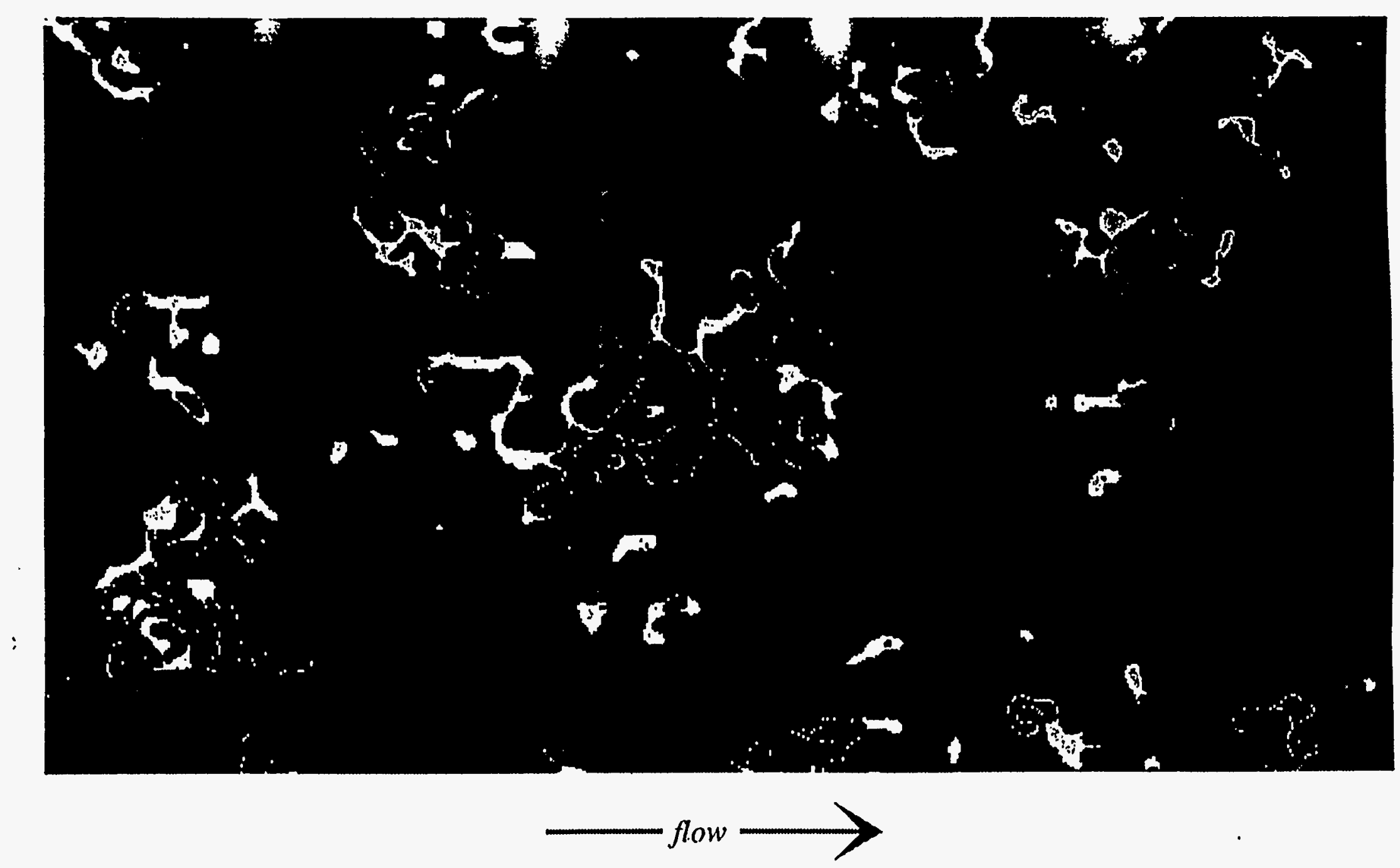

Figure 10a: Order of entrapped phase dissolution: The process of entrapped phase (air) dissolution into the flowing phase (deacrated water) is considered in a horizontal, statistically homogenous (see Figure 3) analog fracture. This image depicts the order of dissolution over a 231 minute time span for a $5.8 \times 10.2 \mathrm{~cm}$ region of the $15 \times 30 \mathrm{~cm}$ experiment; flow is from left to right, at a rate sufficiently high to minimize the formation of a dissolution front. Regions where no change in phase saturation occur are displayed as black (water) and red (air). Regions where dissolution results in the replacement of air by water at intermediate times during the experiment are shown as violet $(t=0-25$ minutes $)$, blue $(t=25-73$ minutes $)$, green $(t=73-141$ minutes $)$, and yellow $(t=141-231$ minutes $)$. Notice that simall entrapped regions dissolve quickly through simple shrinkage, while larger entrapped regions exhibit interfacial recession along cluster appendages with occasional cluster splitting. After becoming sufficiently small, these clusters then dissolve by uniform shrinkage (Glass and Nicholl, 1995). 


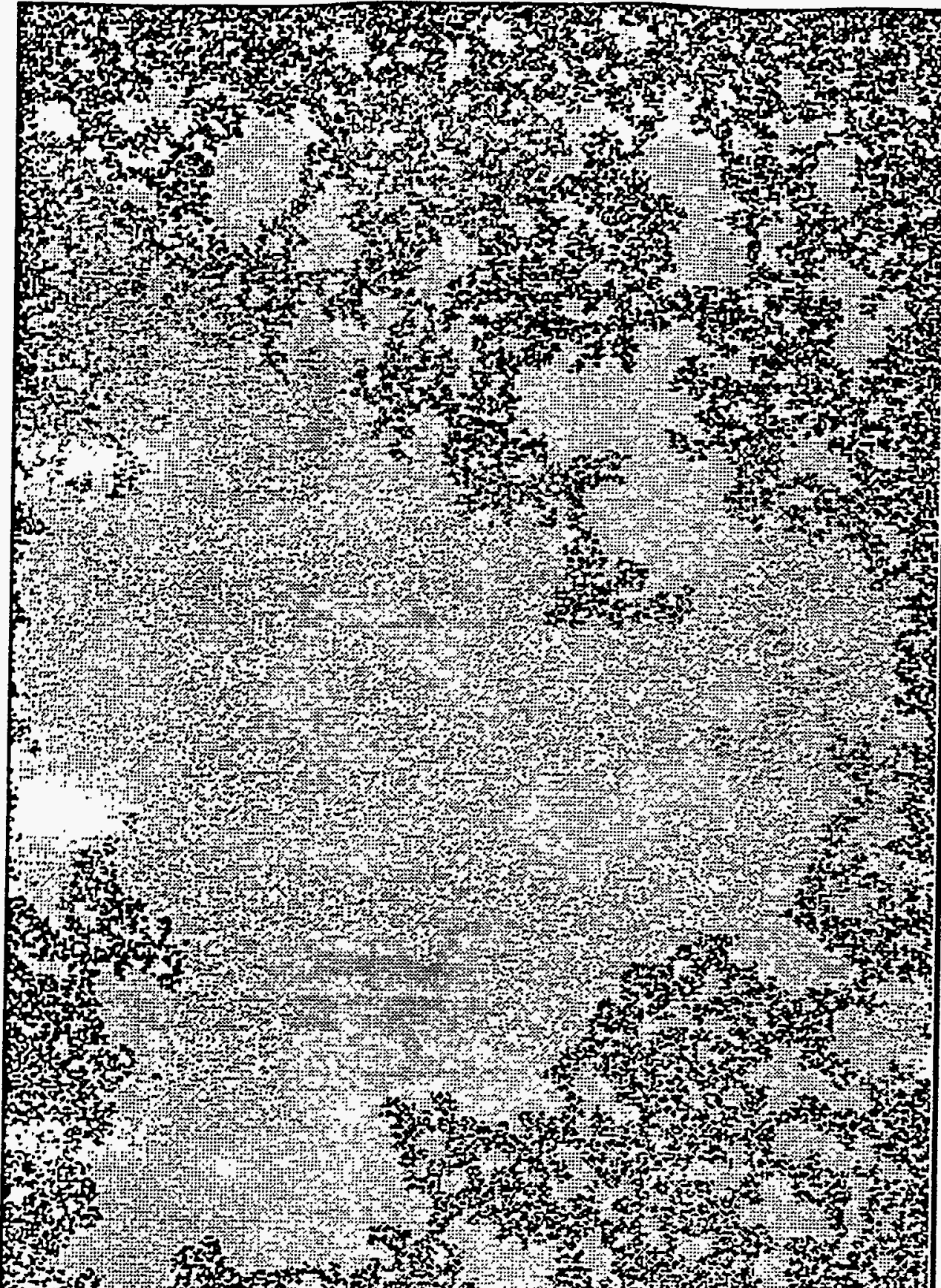

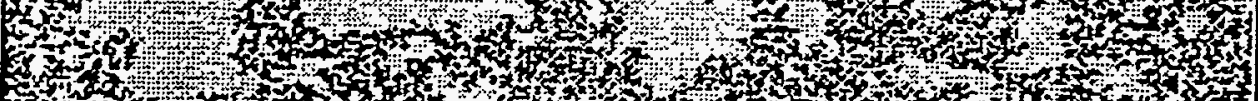

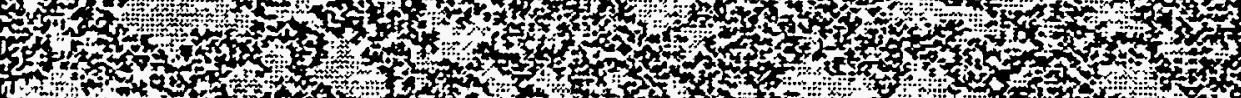

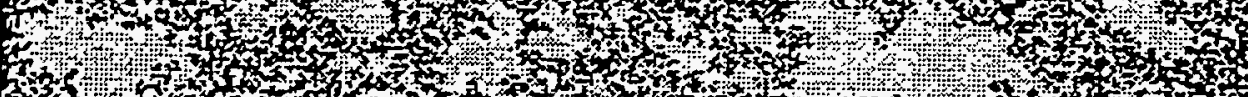

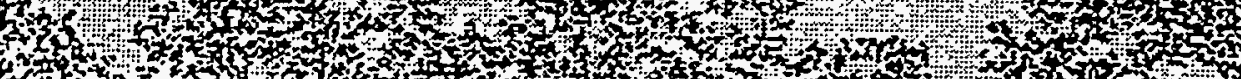

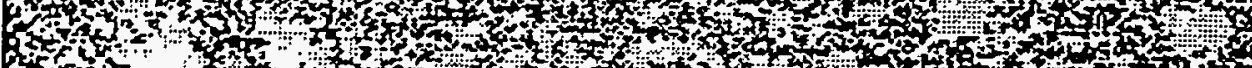

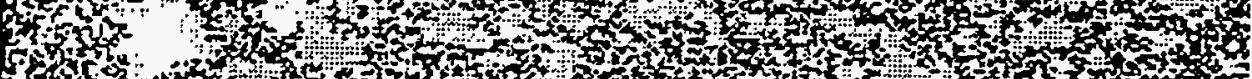

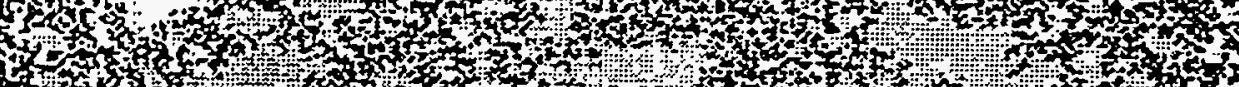

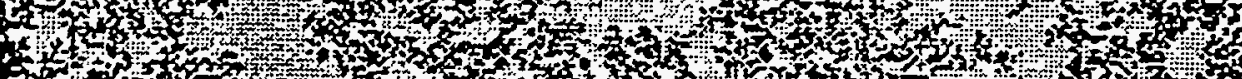
(1)

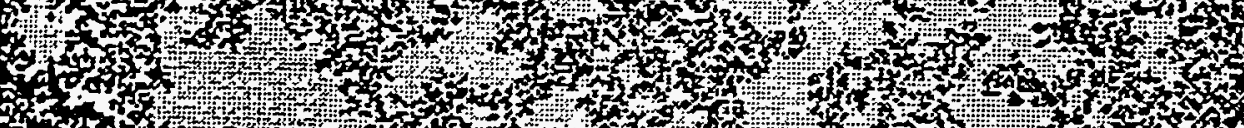

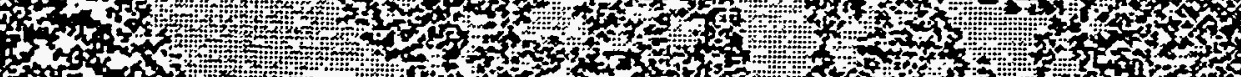
3.

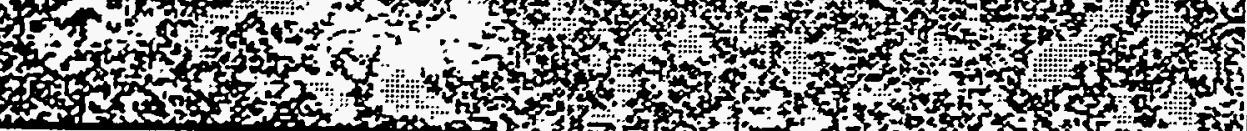

兰

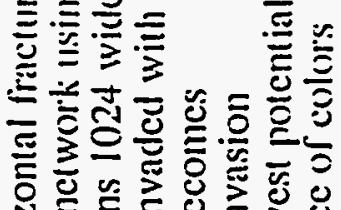

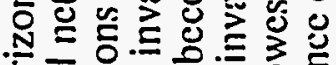

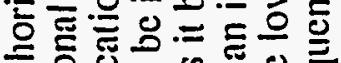

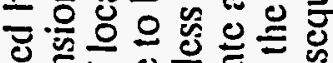

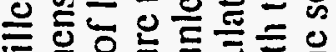

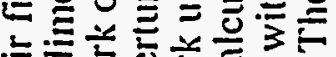

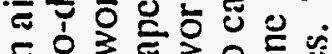
달치웡

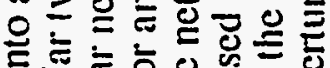

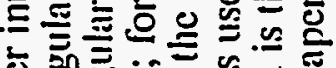

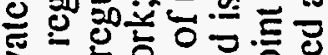

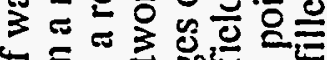
吠

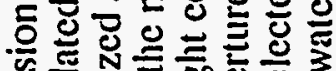

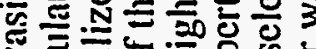

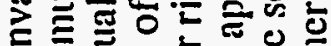

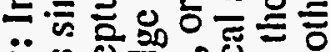

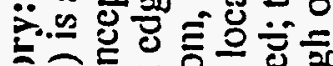

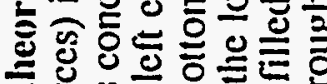
응.

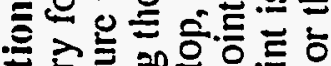

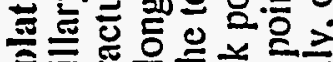

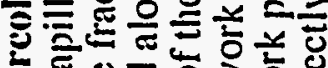

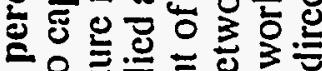

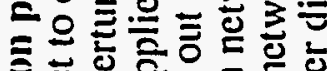

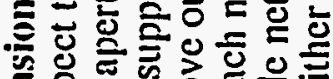

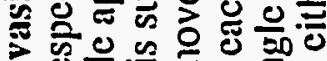

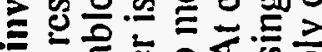
=0

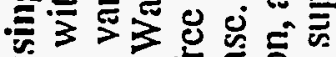
을

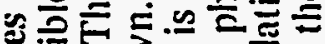

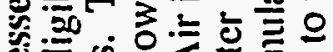

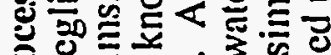

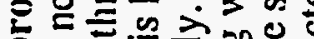
二 管

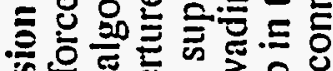

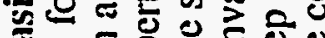

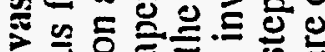
잉을 을

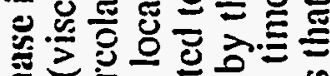

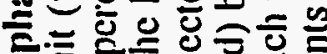

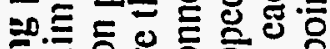

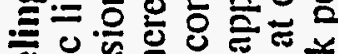

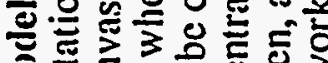
5ू.

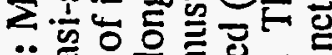
ํํㄹ을

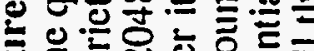

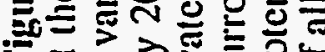

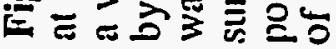




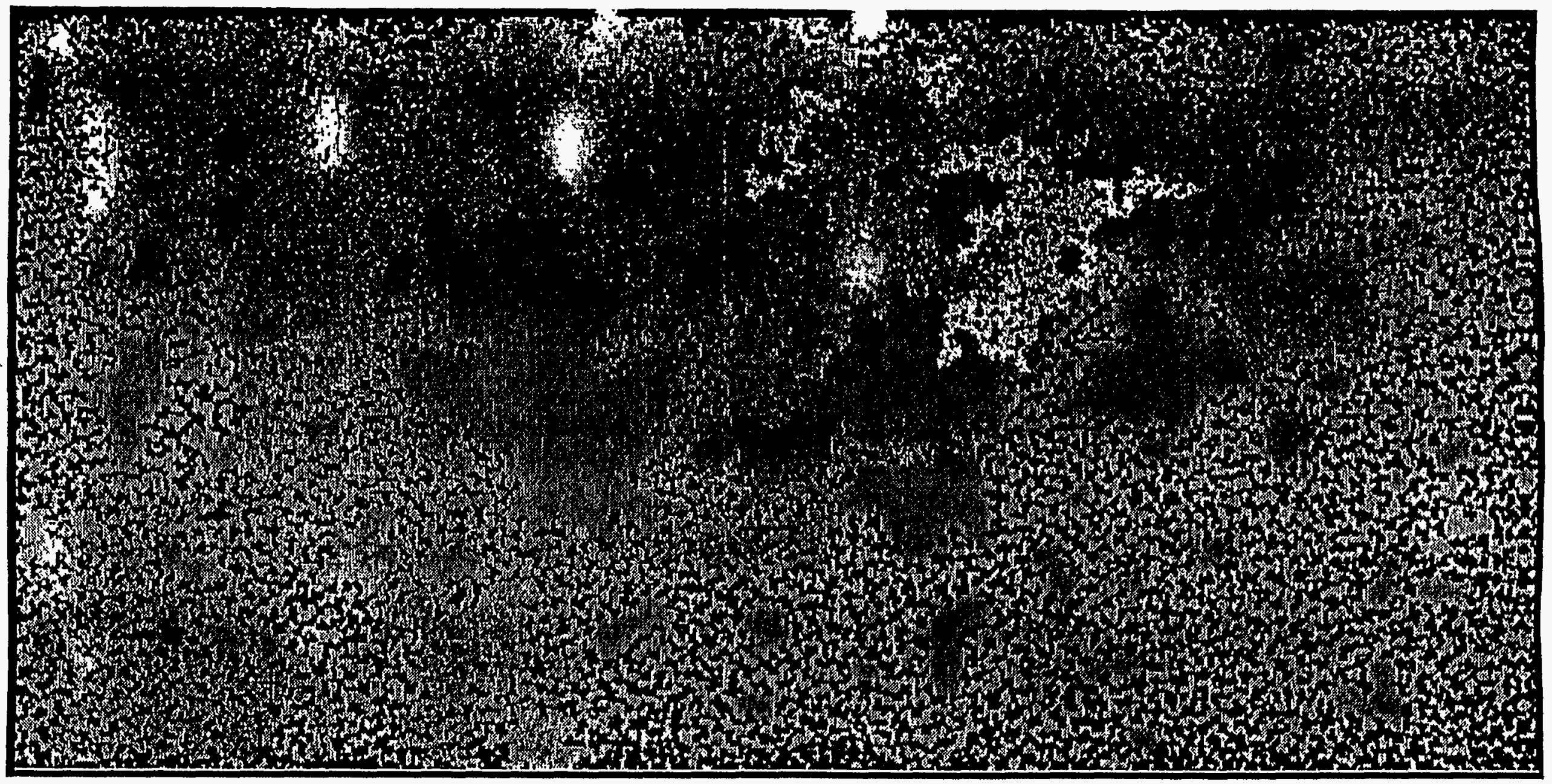

b)

Figure 9 continued: violet-blue-green-yellow-orangc-red show the water invasion sequence, gray depicts the regions of the fracturc where air is entrapped by the invading water phase and black denotes water that fills apertures after the network (fracture) is spanned (percolates). Spanning pressure is achieved in each of the networks at the green to yellow transition. Results of standard percolation theory on a random aperture field (a) and on a 10 by $20 \mathrm{~cm}$ section of the measured analog fracture aperture ficld shown in figure 3 (b). Modifying standard percolation theory to include in-plane interfacial curvature and applying it to the measured analog fracture ficld yiclds phase structures more representative of experiments (c) (Glass, 1993). 